\title{
NANOSATELLITE PROBES IN INTERPLANETARY SPACE: AN AUGMENTED CASSINI MISSION
}

\author{
A Thesis \\ presented to \\ the Faculty of California Polytechnic State University, \\ San Luis Obispo
}

\author{
In Partial Fulfillment \\ of the Requirements for the Degree \\ Master of Science in Aerospace Engineering
}

by

Trent T Voris

July 2017 
(C) 2017

Trent $\mathrm{T}$ Voris

\section{ALL RIGHTS RESERVED}




\section{COMMITTEE MEMBERSHIP}

TITLE: Nanosatellite Probes In Interplanetary Space:

An Augmented Cassini Mission

AUTHOR: Trent T Voris

DATE SUBMITTED: July 2017

COMMITTEE CHAIR: Dr. Jordi Puig-Suari, Ph.D.

Professor of Aerospace Engineering

COMMITTEE MEMBER: Dr. Eric Mehiel, Ph.D.

Professor of Aerospace Engineering

COMMITTEE MEMBER: Dr. Kira Abercromby, Ph.D.

Associate Professor of Aerospace Engineering

COMMITTEE MEMBER: Dr. Ricardo Tubio-Pardavia, Ph.D.

Systems Engineer 


\begin{abstract}
Nanosatellite Probes In Interplanetary Space: An Augmented Cassini Mission Trent $\mathrm{T}$ Voris
\end{abstract}

The exploration of interplanetary space is one of the most challenging and costly ventures in human history. The relatively low amount of information on other sites beyond Earth is largely due to the rarity of effective trajectories as well as the high levels of risk and complexity inherent in innovative space exploration. One solution to this lack of information is the use of deployable satellite probes to help augment the main mission and its instrumentation. This "Mother-Daughter" architecture allows for the low-cost exploration of hazardous sites and numerous points of interest without compromising the primary mission.

While the end goal is the use of nanosatellites on future interplanetary missions, this thesis focuses on an existing interplanetary mission, Cassini. The aim to demonstrate the scientific viability of this "Mother-Daughter" architecture can be achieved by locating numerous unexplored sites that could have been surveyed with a nanosatellite probe onboard Cassini. Each of these potential sites can be expanded into a unique science mission of its own, and in many cases the trajectories can be selected and optimized to better suit the practical design of a nanosatellite in the various interplanetary environments.

Keywords: Architecture, Cassini, CubeSat, Daughter, Interplanetary, Mother, Nanosatellite, Probe, Saturn, Space, Spacecraft, Trajectory 


\section{ACKNOWLEDGMENTS}

Thanks to Dr. Jordi Puig-Suari, my advisor, for working with me to create a thesis that was both interesting and applicable to the future of interplanetary space exploration, and for putting me in contact with the brightest minds in the field of nanosatellites from NASA's Jet Propulsion Laboratory in Pasadena. My gratitude also extends out to Dr. Eric Mehiel for his patience and guidance that allowed me to work on a project that best fit my strengths and passions in Aerospace Engineering. Thanks to the scientists and engineers of NASA's Jet Propulsion Laboratory for allowing me to participate in discussions and studies regarding nanosatellites in interplanetary space. Thanks to my peers at PolySat for showing interest in my results and processes through out this study. Thanks to friends and family for giving me a place to sleep and keeping me sane. Thanks to the baristas of Starbucks on Union, Blossom Hill, Five Cities, and Hermosa Beach for all of the Chai Lattes, Turkey Pesto Sandwiches, and Wi-Fi that allowed me to put in the long hours needed to finish this thesis. 


\section{TABLE OF CONTENTS}

\section{Page}

LIST OF TABLES viii

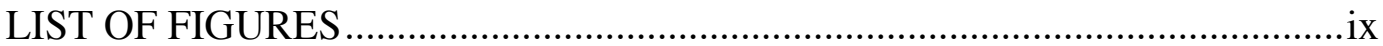

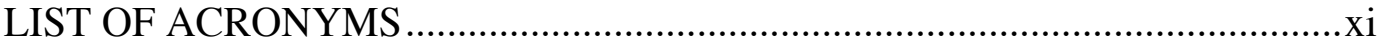

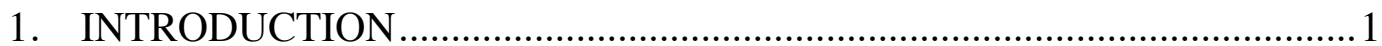

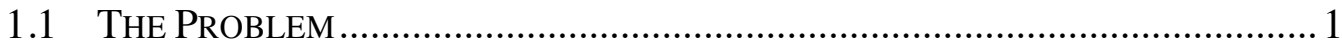

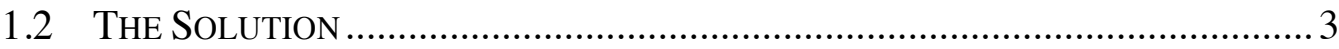

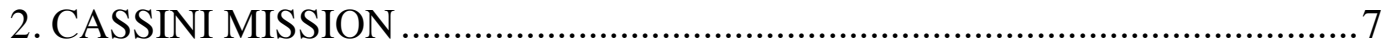

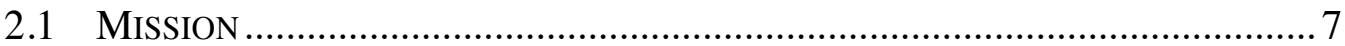

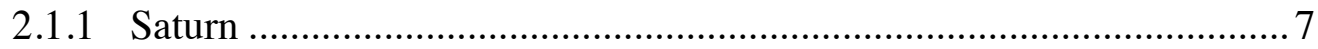

2.1.2 Ring science objectives ..............................................................

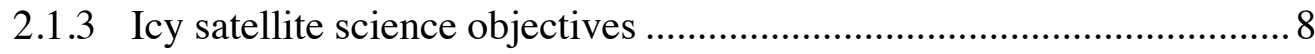

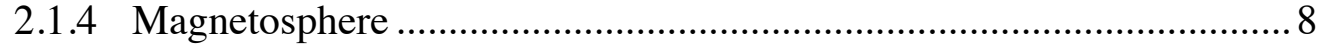

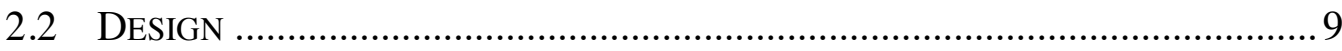

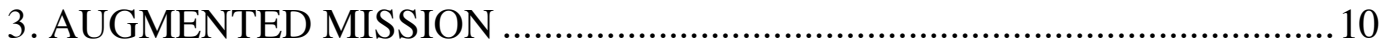

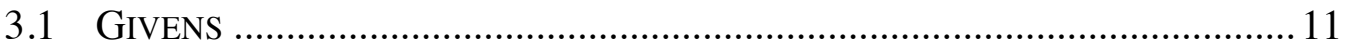

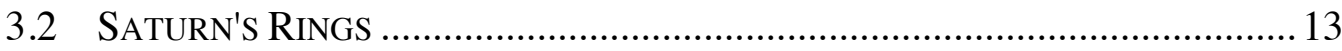

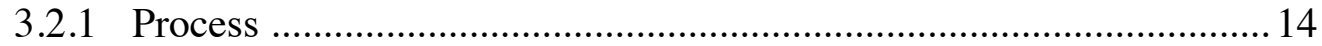

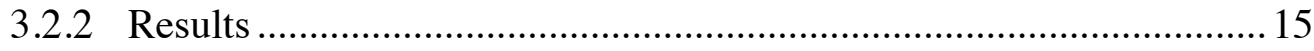

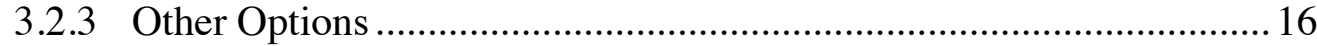

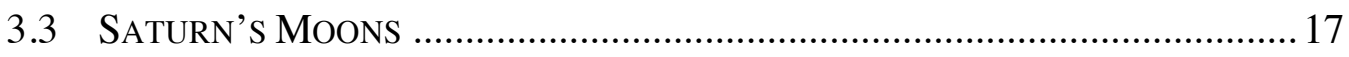




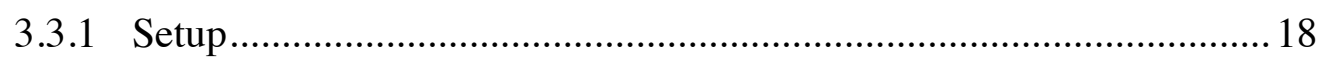

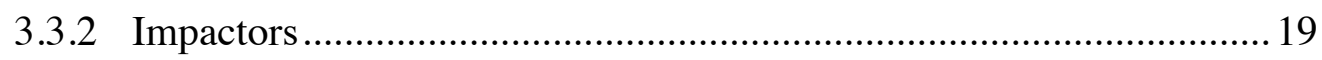

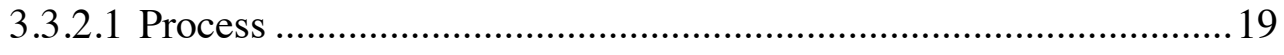

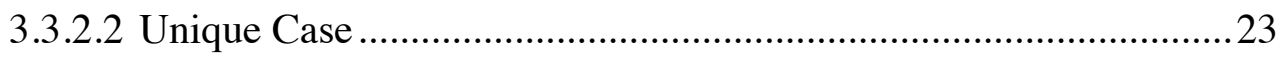

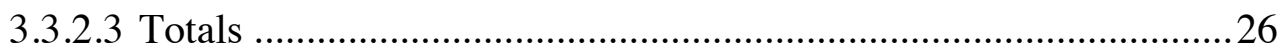

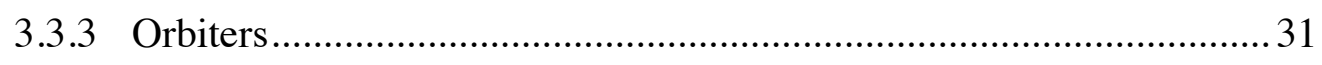

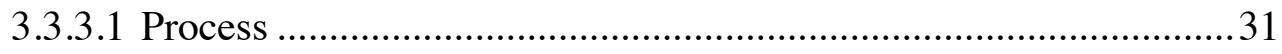

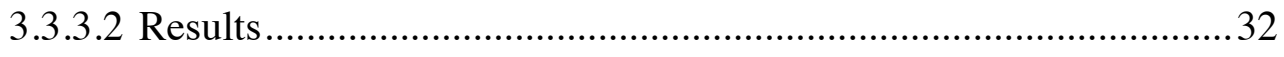

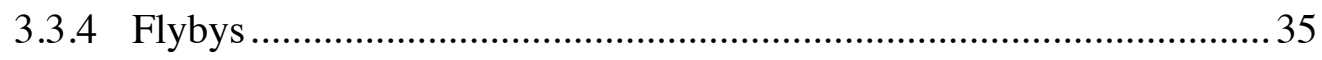

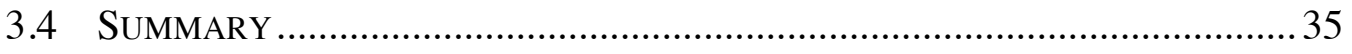

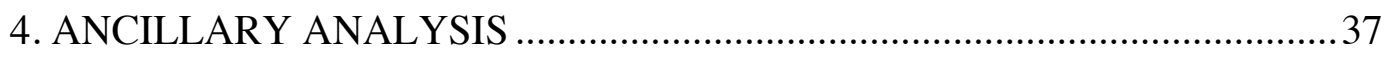

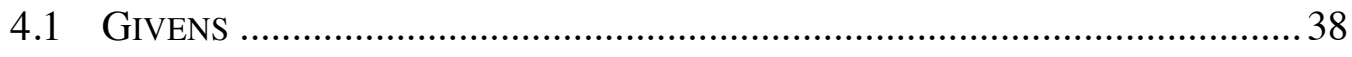

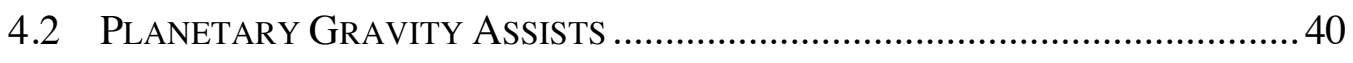

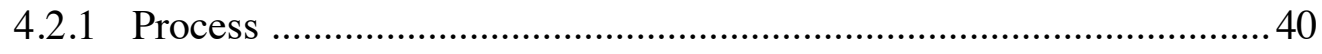

4.2.1.1 Deployment Phase ......................................................................... 40

4.2.1.2 Gravity Assist Phase .....................................................................42

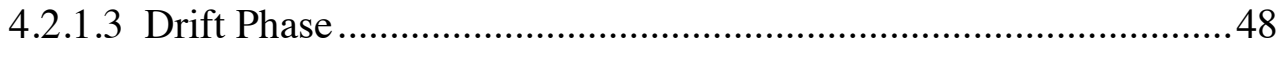

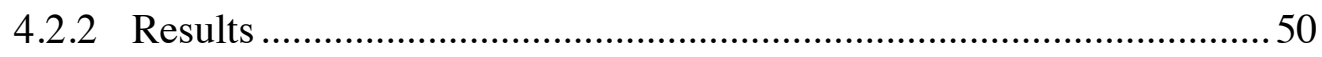

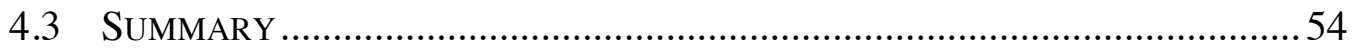

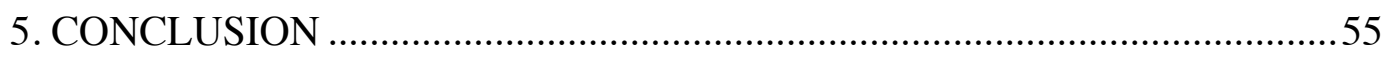

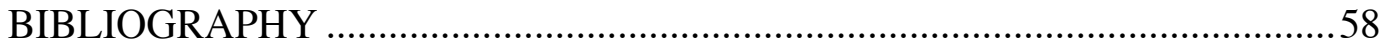

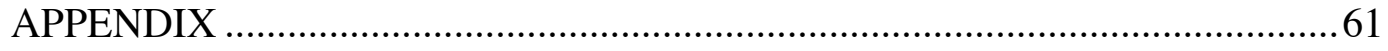




\section{LIST OF TABLES}

Page

Table 1. Missions to the Outer Planets [2] ....................................................... 2

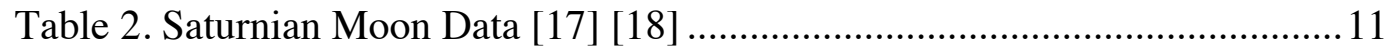

Table 3. Excerpt from Cassini's Tour of the Saturnian System [10] .....................13

Table 4. Saturn Ring Impactor Results [19] ................................................. 16

Table 5. Significant Gravity Wells in the Saturnian System..............................19

Table 6. Excerpt of Moon Trajectory Data ....................................................23

Table 7. Impactor Proof of Concept ...............................................................24

Table 8. Saturn's Moon Exploration Possibilities ...............................................36

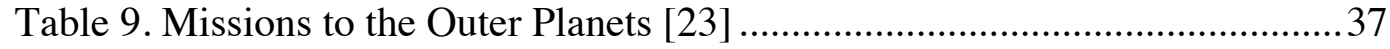

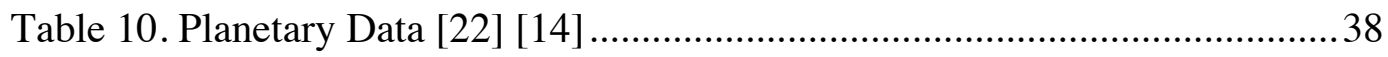

Table 11. Planetary Gravity Assist Parameters [22] ........................................45

Table 12. Augmented Cassini's Planetary Possibilities.....................................54

Table 13. Augmented Cassini Possibilities .......................................................57 


\section{LIST OF FIGURES}

Page

Figure 1. Cosmic Journey [1] ..................................................................

Figure 2. Daughter Nanosatellite Deployment [3] ........................................... 4

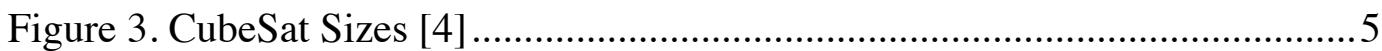

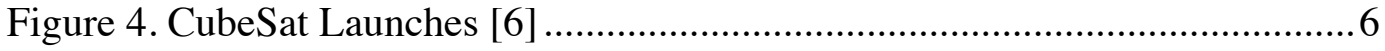

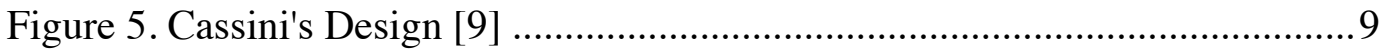

Figure 6. Cassini's Moon Flybys ........................................................... 10

Figure 7. Cassini's Complete Saturnian Trajectory ......................................... 12

Figure 8. Cassini's Specific Orbital Energy ….................................................. 14

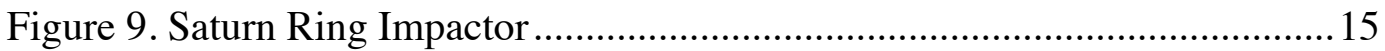

Figure 10. Gravity of the Saturnian System ..................................................... 18

Figure 11. Moon Impactor Example .........................................................2 20

Figure 12. Moon Impactor Example with Variable Delta V ..............................22

Figure 13. Release Time and Initial Delta V Envelope ....................................25

Figure 14. Minimum Delta V to Impact Saturn's Moons ..................................22

Figure 15. Potential Impact Cases (0-700 Cases) ...........................................28

Figure 16. Potential Impact Cases (0-50 Cases) ..............................................29

Figure 17. Potential Impact Cases (Percentage) ............................................... 30

Figure 18. General Moon Orbit Solution ...................................................... 32

Figure 19. Minimum Delta V to Orbit Saturn's Moons .......................................33

Figure 20. Potential Orbit Cases (0-200 Cases) ...................................................34

Figure 21. Potential Orbit Cases (Percentage) .................................................... 34 
Figure 22. Cassini Timeline

Figure 23. Cassini's Interplanetary Trajectory ...............................................39

Figure 24. Daughter Spacecraft Deployment Range ....................................... 41

Figure 25. Simplified Jupiter SOI Entry ....................................................42

Figure 26. Interplanetary to Planetary Frame Shift ........................................ 43

Figure 27. Locus of Possibilities [15] ..........................................................4

Figure 28. Target Circle and Gravitational Sphere of Influence ........................46

Figure 29. Delta V Comparison for Patched Conics ..........................................46

Figure 30. Gravity Assist Locus in the Planet Centered Frame ...........................48

Figure 31. Example Gravity Assist Timeline ..................................................49

Figure 32. Gravity Assist Propagation (15 years) ..........................................50

Figure 33. Venus 1998 Gravity Assist Possibilities ..........................................51

Figure 34. Jupiter 2000 Gravity Assist Possibilities .........................................51

Figure 35. Maximum Inclination from the Ecliptic .......................................52

Figure 36. Maximum Distance from the Sun After 10 Years .............................53 


\section{LIST OF ACRONYMS}

$\Delta \mathrm{V}$ Change in Velocity

AU Astronomical Units

COTS Commercial Off The Shelf

ESA European Space Agency

G Gravity constant

IPEX Intelligent Payload EXperiment

J2000 January 1, 2000 epoch

LCROSS Lunar CRater Observation and Sensing Satellite

NASA National Aeronautics and Space Administration

P-POD Poly-Picosatellite Orbital Deployer

SCET Spacecraft Event Time

SOI Sphere of Influence (gravitational)

U Unit

UTC Coordinated Universal Time

XXM Extended-Extended Mission 


\section{INTRODUCTION}

There is currently a lack of data and knowledge on interplanetary space. This problem is can be traced down to two major roots. The first of these root causes is the fact that interplanetary trajectories are rare, and naturally become scarcer as the number of gravity assists increases. One example, the "Grand Tour" trajectory used by the two Voyager spacecraft in 1977 only happens once every 175 years. The second root restriction on interplanetary information is the risk and complexity inherent in innovative space exploration. Short of changing planetary orbits, many experts are looking to the ever-growing capabilities of small satellites as one possible solution to capitalize on rare orbital opportunities as well as cut down on complexity and risk. The most prominent of these concepts is the Mother-Daughter architecture. This architecture involves attaching small satellites to a larger spacecraft as it navigates through space and then deploying them in order to investigate other points of interest. This concept could drastically increase the amount of in situ information from a single launch.

\subsection{The Problem}

There is so much that humanity does not understand about the solar system. From the atmospheric structures of the ice giants to the radiation environment in interplanetary space, there is a severe lack of knowledge that can only be resolved through increasing the amount of in situ measurements. A lot of this knowledge is imperative for colonizing other planets, searching for extraterrestrial life, and understanding our place in the universe. 


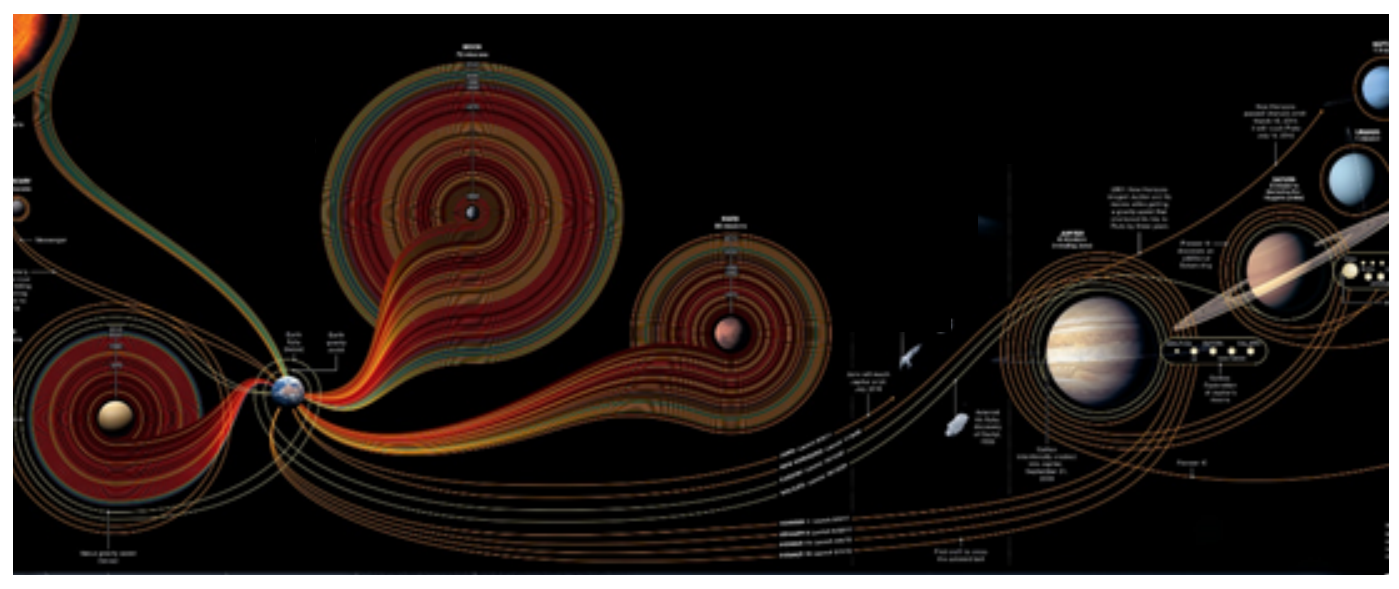

Figure 1. Cosmic Journey [1]

To put it in perspective, Figure 1 shows every interplanetary mission launched, including lunar missions, each as a single line. The most populated body is the Moon followed by Mars and Venus. The scarcity of space data is most prevalent in the outer planets due to the rarity of feasible trajectories and the system complexity required to reach those locations. In fact, there have only been nine missions that passed by any of the outer planets; all of these can be seen on Table 1 below.

Table 1. Missions to the Outer Planets [2]

\begin{tabular}{|c|c|c|c|c|c|}
\hline Spacecraft & Jupiter & Saturn & Uranus & Neptune & Pluto \\
\hline Pioneer 10 & 1973 flyby & & & & \\
\hline Pioneer 11 & 1974 flyby & 1979 flyby & & & \\
\hline Voyager 1 & 1979 flyby & 1980 flyby & & & \\
\hline Voyager 2 & 1979 flyby & 1981 flyby & $\begin{array}{c}1986 \\
\text { flyby }\end{array}$ & $\begin{array}{c}1989 \\
\text { flyby }\end{array}$ & \\
\hline Galileo & $\begin{array}{c}1995-2003 \text { orbit; } \\
1995,2003 \text { atmo. }\end{array}$ & & & & \\
\hline Ulysses & 1992,2004 flyby & & & & \\
\hline $\begin{array}{c}\text { Cassini- } \\
\text { Huygens }\end{array}$ & 2000 flyby & $\begin{array}{c}\text { 2004-now orbit; } \\
\text { 2005 lander }\end{array}$ & & & $\begin{array}{c}2015 \\
\text { flyby }\end{array}$ \\
\hline $\begin{array}{c}\text { New } \\
\text { Horizons }\end{array}$ & 2007 flyby & & & & \\
\hline Juno & 2016-now orbiter & & & & \\
\hline
\end{tabular}

Currently, Voyager 2 is the only manmade spacecraft to gather data at Neptune or Uranus. The models defining the environments of these ice giants are constantly being redefined as more advanced mathematical processes are developed. Any additional data points could easily 
provide significant improvements to our understanding of these environments, and in turn answer some of the bigger questions like how the planets were formed or whether they could support life.

There are so many unexplored areas within our own solar system. By extension there are so many unanswered questions in our solar system. A few of these unanswered questions include:

What do the other objects look like in the Kuiper Belt?

How big is Jupiter's core?

What is the environment of the Oort Cloud?

Are there any asteroids coming toward Earth?

What are all of Saturn's rings made of?

Are there any habitable places in the asteroid belt?

Why does Uranus spin sideways?

Is Neptune warmer than Uranus?

Why do Neptune and Uranus have horizontal magnetospheres?

What does the solar system look like out of plane?

Are there any other planets in the solar system?

\subsection{The Solution}

The simple solution to this interplanetary problem is to capitalize on these rare trajectories by launching numerous spacecraft in each launch window. This simple solution however has some monetary drawbacks; if each spacecraft were on the same magnitude as a typical interplanetary spacecraft, then this solution would involve the use of numerous launch vehicles and billions of extra dollars in costs. To address the resource constraint, a more practical method to launching numerous spacecraft is to shrink the spacecraft down so that they can all fit onto a single launch vehicle. A swarm of small satellites is one architecture considered by professionals at the forefront of space exploration. This is where each spacecraft is on the same magnitude and the loss of any one member would not compromise the entire mission. The other prevalent option, which shall be explored in depth in this thesis, is known as a Mother-Daughter architecture. This Mother-Daughter architecture involves the use of a core interplanetary 
mothership with one or more small daughter spacecraft onboard [2]. Figure 2 shows the International Space Station doing something like this, where it deploys CubeSats stored on board to carry out various missions on Earth orbit.

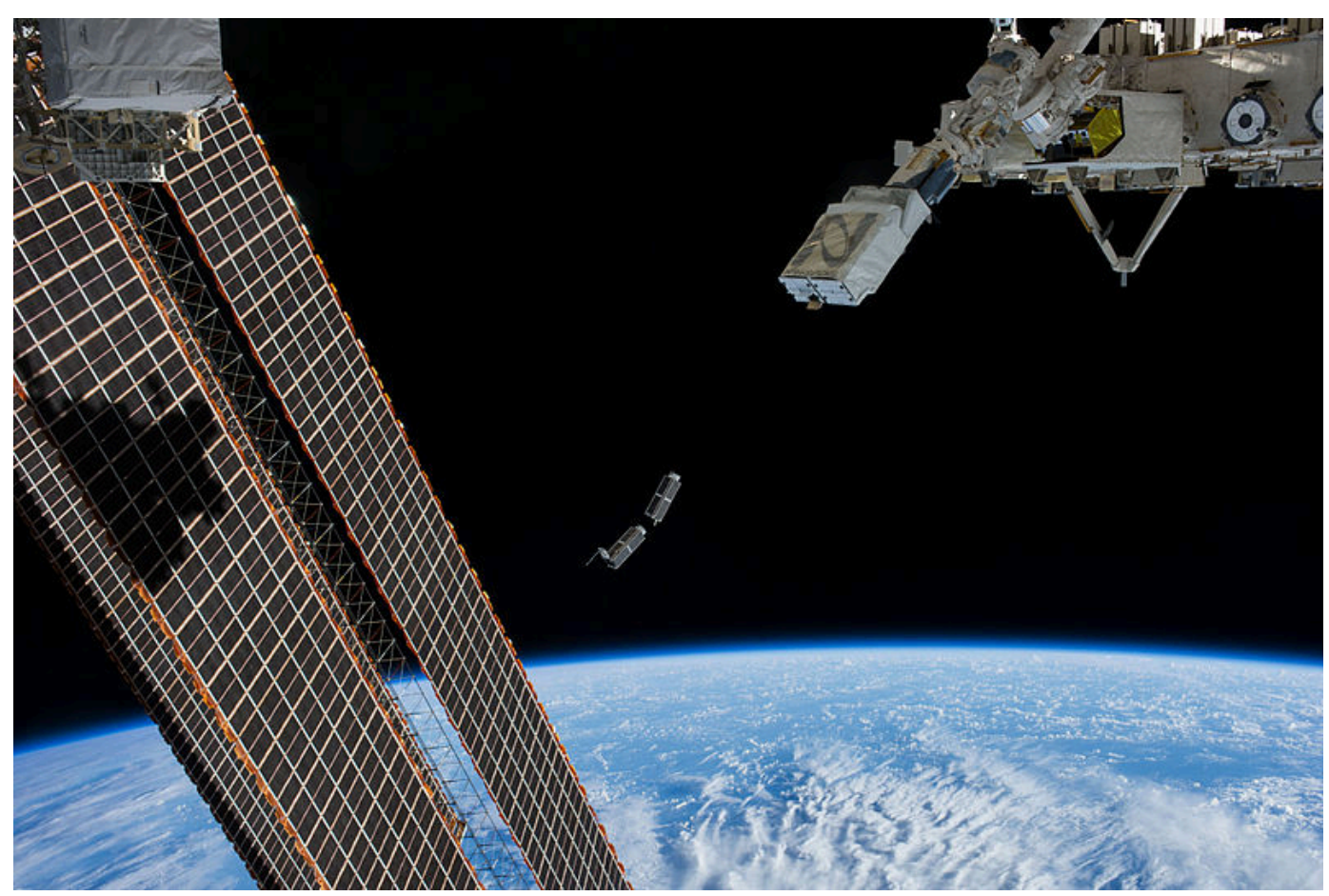

Figure 2. Daughter Nanosatellite Deployment [3]

The Mother-Daughter, rather than the swarm, architecture allows for a simpler design for the small spacecraft and reduces the need for repetitive subsystems. Also, the Mother-Daughter architecture is more likely to be adopted since it is a much smaller deviation from the current status quo of interplanetary exploration than a swarm of small spacecraft. As such, the MotherDaughter architecture is projected as the first step to getting the data needed to better understand the solar system.

The use of small satellites has been gaining traction since the turn of the century [3]. This growth is due largely in part to the rapid growth in microelectronics. With nanosatellites, many of the components that are typically custom made for satellites can now be effectively replaced by smaller and cheaper commercial off the shelf (COTS) components. This new design has since revolutionized the space industry by challenging the popular "failure is not an option" mentality adopted by many of the larger space companies. 
The CubeSat Standard is one of the most prevalent nanosatellite standards partially due to its simplicity. The high level concept of such a CubeSat consists of breaking down a satellite into small 10 centimeter cubes known as Units (or U's for short). Figure 1 below shows a few of the various sizes possible with the CubeSat Standard. These standard sizes allow for a generic deployment mechanism like the CubeSat P-POD, which can carry any combination of 3 Us into orbit by ride sharing with a larger satellite. The remaining requirements in the CubeSat Standard exist almost entirely to ensure the safety and success of the launch vehicle and the primary payload that are granting the CubeSat passage to orbit.

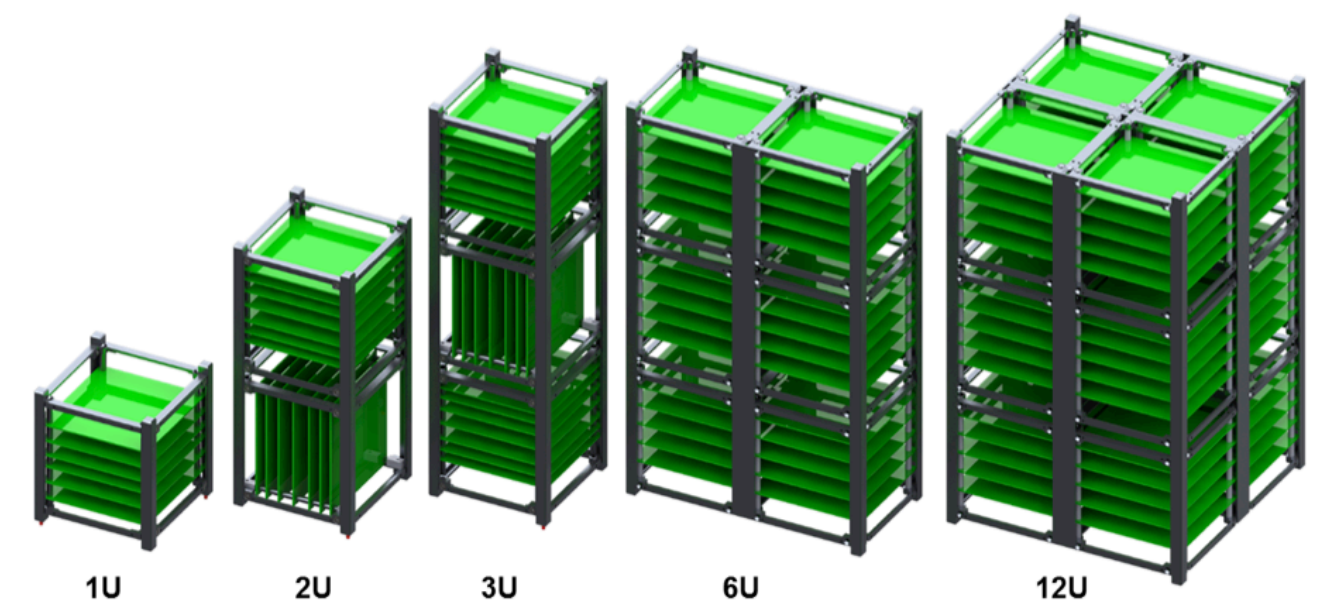

Figure 3. CubeSat Sizes [4]

At the time this is being written there have been 461 CubeSat Missions [5]. The growth can be seen in Figure 4, which illustrates the increase of CubeSat on orbit over time. This increase serves as a good index by which to judge the growth of small satellite technology. The rapid engineering process behind CubeSats has allowed the nanosatellite technology to make leaps and bounds over the technology used on the larger enterprises. One example of this development can be seen through the mission success rate, in the first five years (2000-2004) there was a $23 \%$ success rate in CubeSat missions, and now in the most recent five years (2012-2016) that success rate improved to $69 \%$ [5]. These CubeSats have tested over a hundred new technologies ranging from new image processing software (IPEX) to the implementation of solar sails (LightSail). Many of the necessary components for any type of mission are already available off the shelf. 


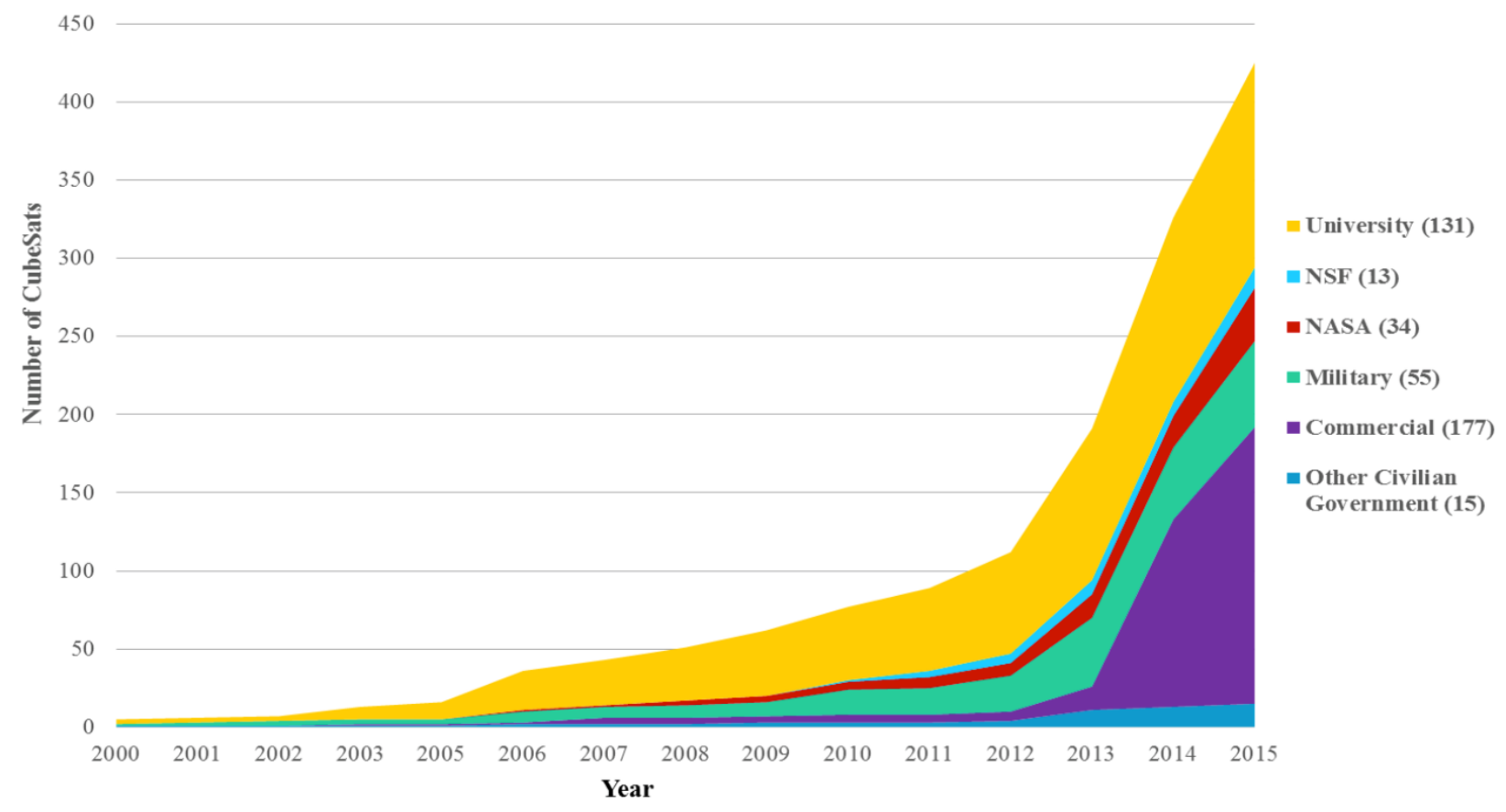

Figure 4. CubeSat Launches [6]

The other big selling point for nanosatellite technology is just that, the cost. PhoneSat, currently the cheapest satellite on orbit cost NASA about $\$ 3,500$ to build [7]. Many of the new innovative CubeSats cost around $\$ 1,000,000$. While CubeSats vary significantly in cost an average for a standard terrestrial mission is on the order of a $\$ 100,000$. To put these numbers into perspective, Cassini cost the United States around \$2.6 billion [8].

Due to its negligible size, cost, and impact on the primary spacecraft the CubeSat Standard is a leading candidate when looking at future Mother-Daughter architectures for the interplanetary environment. There are other prevalent sizes and standards that have been conceptualized and implemented each with their own added benefits and costs. While the CubeSat Standard is a robust solution, each of these options should be considered and evaluated for any given mission and its requirements of the daughter spacecraft. 


\section{CASSINI MISSION}

While the processes used in the following analysis can pertain to any interplanetary mission past or present, the Cassini mission was selected as a template for analyzing the benefits of a Mother-Daughter architecture. Using Cassini as the mothership afforded a well-understood case from which to base the analysis of potential daughter missions. With Cassini the driving purpose was to explore as much of the Saturnian System as possible. Cassini's trajectory though complicated, is now well defined and publicly available making it an ideal case for exploring the potential of the interplanetary Mother-Daughter architecture.

\subsection{Mission}

According to the European Space Agency, Cassini's prime mission can be broken down into four distinct categories: Saturn, the rings, the moons, and the magnetosphere:

\subsubsection{Saturn}

- Determine the vertical structure of the atmosphere, in particular, how its composition, cloud properties, density, and temperature vary with height;

- Understand the horizontal motions of the atmosphere: its waves, eddies, and storms - where they are located and how they form, grow, evolve, and dissipate;

- Determine the deep structure of the atmosphere, how it rotates, and how it relates to the upper atmosphere;

- Study how the atmosphere varies with time, both on short (daily) and long (seasonal) time scales;

- Investigate the relationship between the ionosphere, the magnetic field, and the plasma environment;

- Investigate the sources of lightning.

\subsubsection{Ring science objectives}

- Map the composition and size distribution of ring material;

- Study the configuration of the rings and the dynamic processes responsible for their structure;

- Investigate the relationships between the rings and the embedded moons;

- Search for new ring-embedded moons;

- Study the interaction between the rings and Saturn's magnetosphere, ionosphere, and atmosphere. 


\subsubsection{Icy satellite science objectives}

- Map their surface geology and composition and determine their geologic histories;

- Determine the physical processes responsible for the surface and subsurface structure;

- Determine their bulk compositions and internal structure;

- Investigate their interactions with Saturn's magnetosphere and ring system.

\subsubsection{Magnetosphere}

- Determine the global configuration and dynamics of hot plasma in the magnetosphere of Saturn through energetic neutral particle imaging of ring current, radiation belts, and neutral clouds;

- Study the sources of plasmas and energetic ions through in situ measurements of energetic ion composition, spectra, charge state, and angular distributions;

- Search for, monitor, and analyze magnetospheric substorm-like activity at Saturn;

- Use imaging and composition studies to determine the magnetosphere- satellite interactions at Saturn, and understand the formation of clouds of neutral hydrogen, nitrogen, and water products (such as protons, oxygen atoms or hydroxyl radicals);

- Study how satellite surfaces and atmospheres are modified due to plasma and radiation bombardment;

- Study Titan's cometary interaction with Saturn's magnetosphere (and the solar wind) via high-resolution imaging and in situ ion and electron measurements;

- Measure the high energy (Ee $>1 \mathrm{MeV}$, Ep $15 \mathrm{MeV}$ ) particle component in the inner $(\mathrm{L}<5 \mathrm{RS})$ magnetosphere to assess cosmic ray albedo neutron decay (CRAND) source characteristics;

- Investigate the absorption of energetic ions and electrons by the satellites and rings in order to determine particle losses and diffusion processes within the magnetosphere;

- Study magnetosphere-ionosphere coupling through remote sensing studies of the aurora and in situ measurements of precipitating energetic ions and electrons [10].

These objectives are not unlike the objectives of other interplanetary missions like Juno,

Voyager, and Galileo, so it stands to reason that in the near future other interplanetary missions

will include similar directives. 


\subsection{Design}

Launched on October 15, 1997, the Cassini mission is projected to last almost two decades in space, six of these years were spent in transit with the remaining 14 years exploring the Saturnian system. Cassini is currently scheduled to impact Saturn on September 15, 2017 [12].
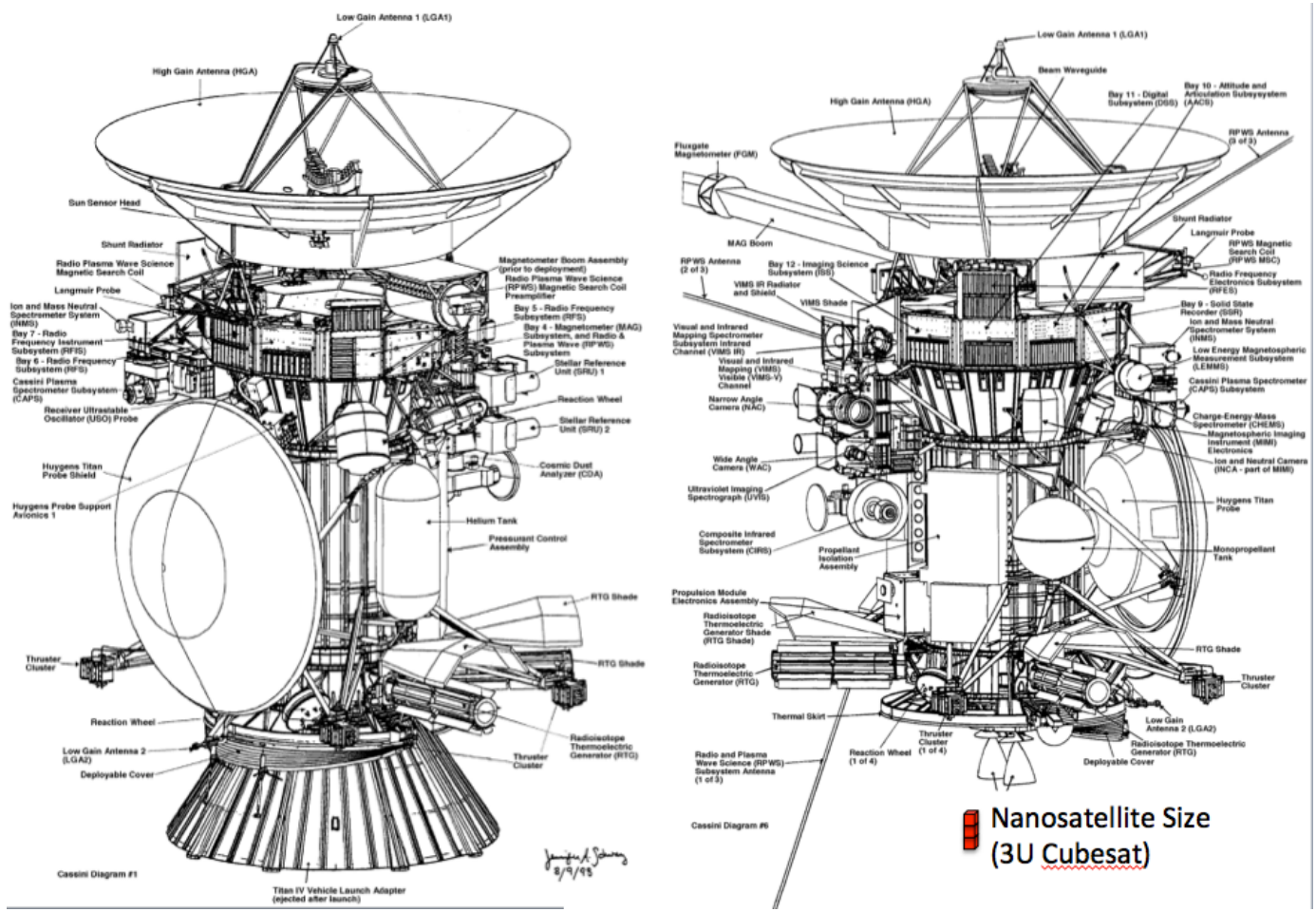

Figure 5. Cassini's Design [9]

Figure 5 above shows the schematics for a fully developed Cassini. A $3 \mathrm{U}$ CubeSat, the most common small satellite on orbit is shown in red for scale. According to NASA Jet Propulsion Laboratory, the mission costs at end of life will be:

$\$ 1.422$ billion pre-launch development; $\$ 710$ million mission operations; $\$ 54$ million tracking; \$422 million launch vehicle; \$500 million ESA; \$160 million ASI; total about $\$ 3.27$ billion, of which U.S. contribution is $\$ 2.6$ billion and European partners' contribution $\$ 660$ million

As mentioned earlier, the average cost of an Earth orbiting CubeSat is around $\$ 100,000$, which is four orders of magnitude less. While the addition of any small satellites will affect certain budgets in the design process, the size of Cassini as well as its cost budget helps to demonstrate the insignificance of a nanosatellite daughter stowed onboard. 


\section{AUGMENTED MISSION}

Since reaching the Saturnian System on July 1, 2004 [8], Cassini has performed a total of 718 flybys at the time this is being written of Saturn's 23 major moons. Many of its discoveries with regard to the moons of Saturn have raised interest in further exploration especially in Enceladus and Titan [8]. Cassini also measured and recorded data on Saturn and its rings as a major part of its primary mission. The final mission for Cassini, its Extended-Extended Mission (XXM) is for Cassini to impact Saturn on September 15, 2017 in order to gain more information on the atmospheric structure of Saturn that can only be obtained in situ [16].

Almost all of the data gathered on Saturn's moons and environment come from this single Cassini mission. While Cassini has been an undeniably successful mission so far, some fundamental issues arise in terms of data analysis and mathematical modeling from using one satellite for the data generation. Even given Cassini's intricate trajectory within Saturn's gravitational sphere of influence, Cassini only gathered significant data points on Titan with 188 flybys, while the other moons remain poorly defined as seen in Figure 6 below. While Cassini did calibrate its instruments during its planetary flybys, the fact that it only flew one of each type payload means that some of the recorded data could be off by an unknown amount and instrument fidelity is hard to determine when there are no other benchmarks to verify perfomance against.

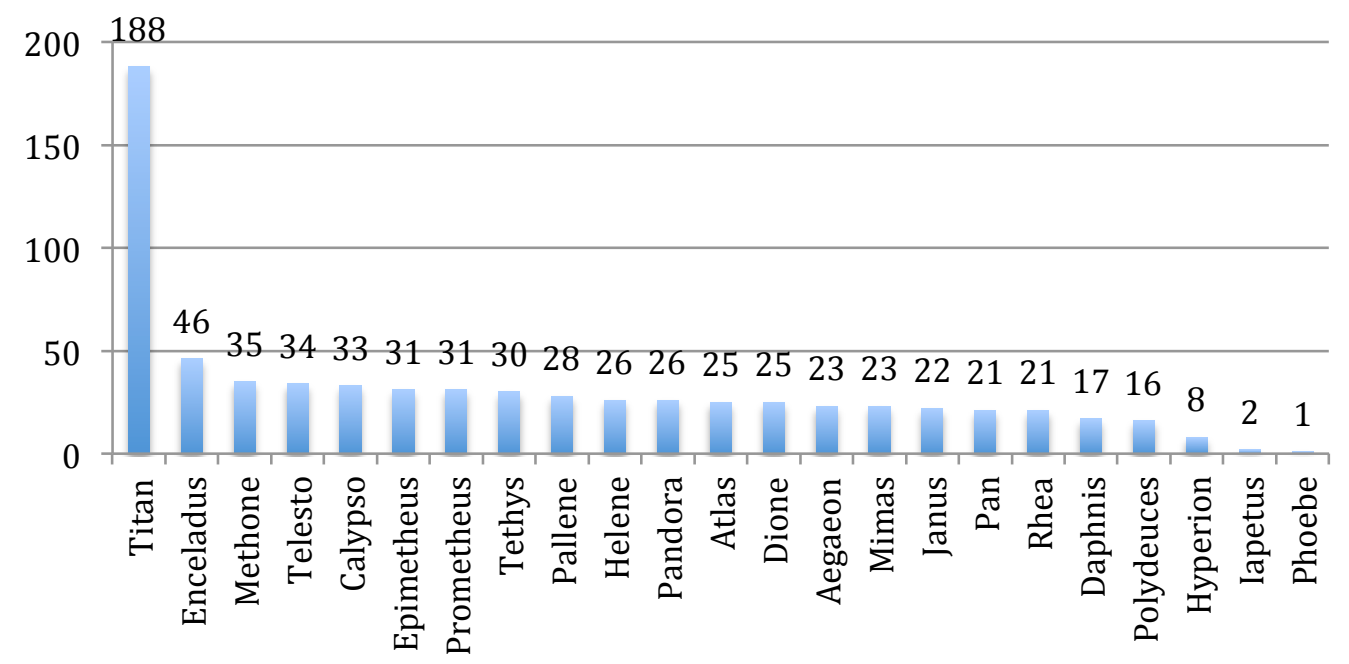

Figure 6. Cassini's Moon Flybys 
This chapter will explore the possibility of using daughter spacecraft to collect data on other points of interest within the Saturnian System. This general analysis aims to reveal the possibilities for impact, orbit, and atmospheric flyby trajectories within the Saturnian System in order to better direct and allow for more focused studies on specific cases depending on the desires and trade offs of the user.

\subsection{Givens}

Table 2 below contains all of the information necessary for the analysis of the Saturnian System. The diameter in kilometers is the maximum diameter of each moon. This is especially important for the smaller moons like Aegaeon, which are significantly less spherical. The mass in kilograms for each of the large moons was determined using precise gravitational measurements and can be found in various papers while the smaller moons were approximated assuming a standard density of $1.3 \mathrm{~g} / \mathrm{cm}^{3}$. This approximation is suitable for the smaller moons since the mass of each moon is only used for determining gravitational spheres of influences, for which the smaller moons are significantly too small to posses their own. The distance from Saturn is simply the distance measured from the center of Saturn to the center of each moon in kilometers.

Table 2. Saturnian Moon Data [17] [18]

\begin{tabular}{|c|c|c|c|}
\hline Name & Diameter $(\mathrm{km})$ & Mass $(\mathrm{kg})$ & $\begin{array}{c}\text { Distance from } \\
\text { Saturn }(\mathrm{km})\end{array}$ \\
\hline Saturn & 120536 & $5.6832 \times 10^{26}$ & 0 \\
\hline Aegaeon & 0.5 & $1.00 \times 10^{11}$ & 167500 \\
\hline Atlas & 30 & $6.60 \times 10^{15}$ & 137670 \\
\hline Calypso & 21 & $6.30 \times 10^{15}$ & 294619 \\
\hline Daphnis & 7.6 & $8.40 \times 10^{13}$ & 136505 \\
\hline Dione & 1123 & $1.10 \times 10^{21}$ & 377396 \\
\hline Enceladus & 504 & $1.10 \times 10^{20}$ & 237950 \\
\hline Epimetheus & 116 & $5.27 \times 10^{17}$ & 151422 \\
\hline Helene & 35.2 & $2.45 \times 10^{16}$ & 377396 \\
\hline Hyperion & 270 & $5.62 \times 10^{18}$ & 1481010 \\
\hline Iapetus & 1470 & $1.80 \times 10^{21}$ & 3560820 \\
\hline Janus & 179 & $1.90 \times 10^{18}$ & 151472 \\
\hline Methone & 3.2 & $2.00 \times 10^{13}$ & 194440 \\
\hline Mimas & 396 & $4.00 \times 10^{19}$ & 185404 \\
\hline Pallene & 5.0 & $5.00 \times 10^{13}$ & 212280 \\
\hline Pan & 28 & $4.95 \times 10^{15}$ & 133584 \\
\hline Pandora & 81 & $1.37 \times 10^{17}$ & 141720 \\
\hline Phoebe & 213 & $8.29 \times 10^{18}$ & 12869700 \\
\hline
\end{tabular}




\begin{tabular}{|c|c|c|c|}
\hline Polydeuces & 2.6 & $3.00 \times 10^{13}$ & 377396 \\
\hline Prometheus & 86 & $1.60 \times 10^{17}$ & 139380 \\
\hline Rhea & 1527 & $2.30 \times 10^{21}$ & 527108 \\
\hline Telesto & 25 & $9.41 \times 10^{15}$ & 294619 \\
\hline Tethys & 1062 & $6.20 \times 10^{20}$ & 294619 \\
\hline Titan & 5150 & $1.35 \times 10^{23}$ & 1221930 \\
\hline
\end{tabular}

Figure 7 below is an image of the Saturnian system as well as Cassini's entire "ball of yarn" trajectory. The image below was generated using MathWorks' MatLab in conjunction with NASA's Spacecraft Planet Instrument Camera-matrix Events (SPICE) software and Cassini dataset, which can be found in the NASA Navigation and Ancillary Information Facility (NAIF) database [15]. This complex weave of various orbital maneuvers and flybys led the analysis to be broken down to focus on each individual flyby rather than attempting to send nanosatellites probes on complex and precise orbital trajectories.

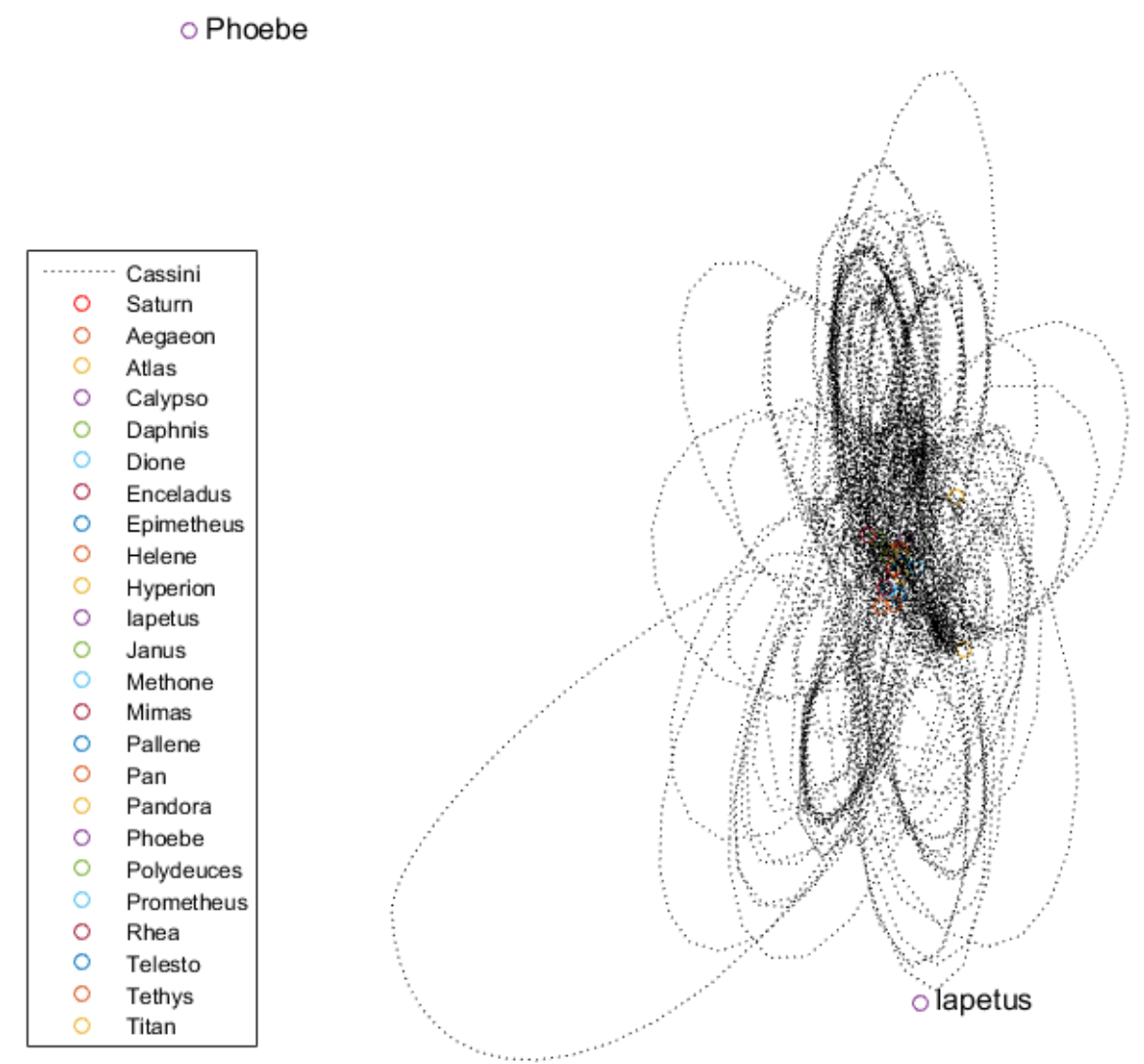

Figure 7. Cassini's Complete Saturnian Trajectory 
Table 3 shows a small excerpt from a large table of flybys performed or to be performed by Cassini as well as some of the flyby's parameters. This table can also be found within the NAIF database [15]. When used in conjunction with the "ball of yarn" state vectors, this table allows for the analysis to focus in on one flyby at a time and then compile the results. The major parameters used from this table were the Moon, Epoch, and the Spacecraft Event Time (SCET). The Moon parameter allowed the analysis to focus on a single target rather than propagating all 23 moons simultaneously for the whole mission. The Epoch and the SCET were also used to help limit the scope of each case. The altitude and speed in kilometers and kilometers per second respectively were used preliminarily as a method of checking the validity of the results, but like the rest of the remaining parameters were not directly utilized.

Table 3. Excerpt from Cassini's Tour of the Saturnian System [10]

\begin{tabular}{|c|c|c|c|c|c|c|c|c|c|}
\hline Rev & Name & Moon & Epoch & $($ SCET $)$ & DOY & Alt $(\mathrm{km})$ & in/out & $\begin{array}{c}\text { Speed } \\
(\mathrm{km} / \mathrm{s})\end{array}$ & $\begin{array}{c}\text { Phase } \\
(\mathrm{deg})\end{array}$ \\
\hline 128 & $128 \mathrm{PO}$ & Polydeuces & $2010-$ Mar-21 & $4: 47$ & 80 & 30029 & out & 10.2 & 70 \\
\hline 129 & $129 \mathrm{CA}$ & Calypso & $2010-$ Apr-07 & $8: 00$ & 97 & 97950 & in & 11.7 & 69 \\
\hline 129 & $129 \mathrm{EP}$ & Epimetheus & $2010-$ Apr-07 & $12: 46$ & 97 & 61715 & in & 2.4 & 89 \\
\hline 129 & $129 \mathrm{JA}$ & Janus & $2010-$ Apr-07 & $13: 44$ & 97 & 74603 & out & 2.8 & 40 \\
\hline 129 & $129 \mathrm{MI}$ & Mimas & $2010-A p r-07$ & $13: 47$ & 97 & 97429 & out & 10.3 & 124 \\
\hline 129 & $129 \mathrm{TE}$ & Tethys & $2010-$ Apr-07 & $14: 58$ & 97 & 70779 & out & 6.4 & 103 \\
\hline
\end{tabular}

\subsection{Saturn's Rings}

A major area of interest in the Cassini mission was Saturn's system of rings. They were studied in depth from a distance using Cassini's ranged instrumentation and from telescopes here on Earth; however, there are a few scientific objectives that can only be achieved by flying through the rings and gathering samples. This includes gathering samples and evaluating the structure. In situ sampling of the rings incurs a high level of risk to Cassini's main mission, but the use of a disposable probe spacecraft could provide key information that would otherwise jeopardize the entire mission. The potential deployment of multiple disposable probes drastically reduces the risk and increases the scientific potential since data would be gathered from multiple locations in the ring system simultaneously. The potential of deploying a daughter spacecraft from Cassini's trajectory will be explored in this section by first minimizing energy of deployment and 
then using a simple antivelocity impulse to drop the daughter spacecraft into an array of impact trajectories with Saturn's ring system.

\subsubsection{Process}

The primary process chosen to impact the rings of Saturn started with locating the minimum in the specific orbital energy of Cassini using Equation 1. Specific Orbital Energy.

\section{Equation 1. Specific Orbital Energy [11]}

$$
E=\frac{v^{2}}{2}-\frac{\mu}{r}
$$

Equation 1. Specific Orbital Energy was applied to Cassini over the entire mission time to find a rough estimate of the local minimum. The minimum point was then used to "zoom in" on the area of lowest energy as seen in Figure 8 below in order to increase the fidelity of the potential transfer. This "zoomed" area was used as the range to locate the optimal starting state for a ring impactor. This ideal starting state was found by introducing an impulse in the antivelocity direction for every point in time within the "zoomed" range. The point that exhibited the greatest decrease in periapse can be seen in Figure 8 below as a red circle.

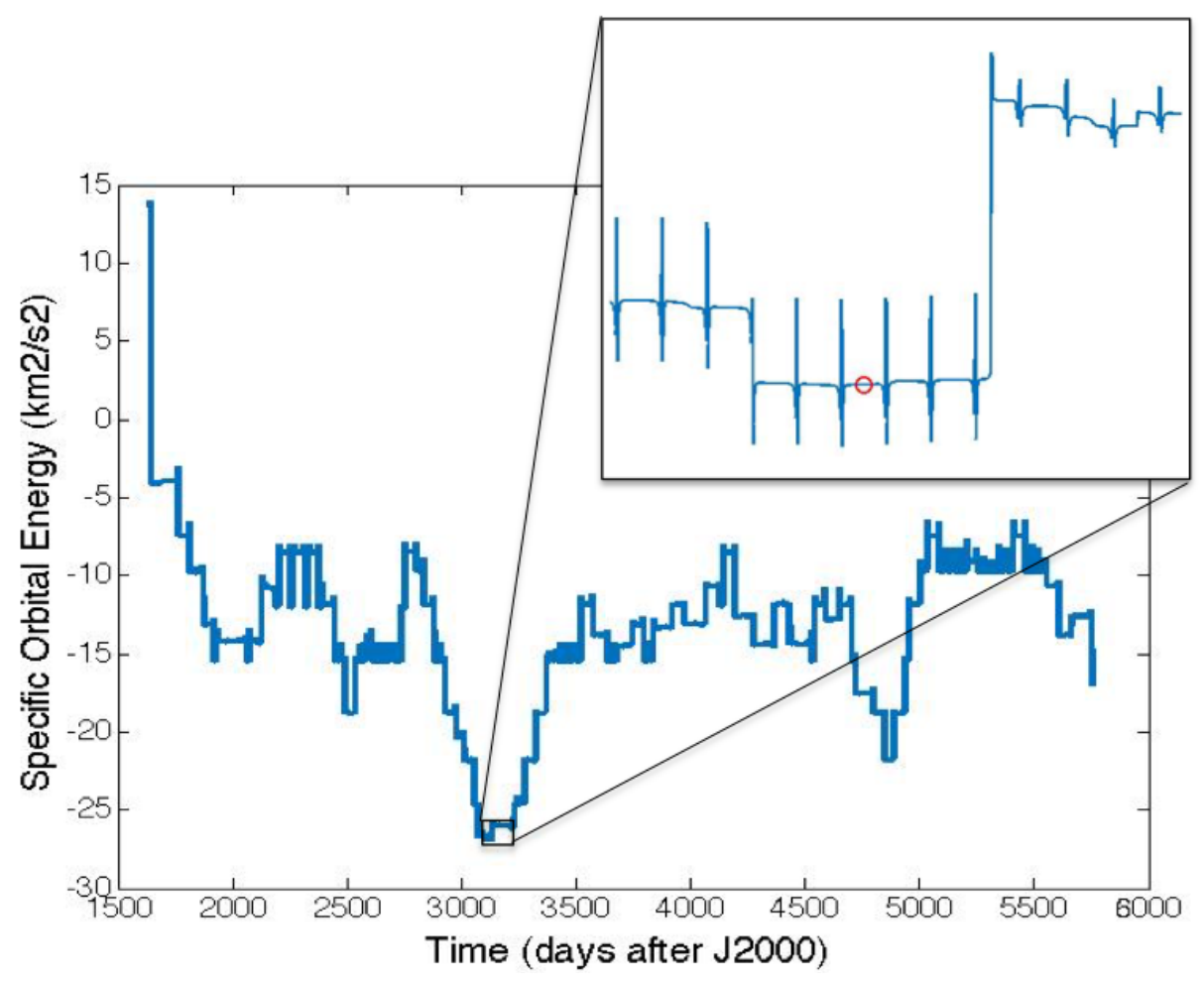

Figure 8. Cassini's Specific Orbital Energy 
The time indicated by the red circle, July 10, 2008, was used to find the instantaneous state of Cassini with respect to Saturn and was used as the deployment point for a range of Delta $\mathrm{V}$ maneuvers. Because the Delta V maneuvers were assumed to be instantaneous, the analysis started with an array of states varying in velocity magnitude but otherwise identical. The varying states, all with the same position, were then propagated forward in time and terminated if they impacted Saturn itself. The altitude of the spacecraft as it crossed the rings was recorded by taking the total magnitude of the new position vector when the magnitude in the $\mathrm{z}$ direction was zero. The $\mathrm{z}$ direction in the Saturn centric frame is by definition normal to the equator of Saturn and by extension the rings. The purpose was to deduce whether a maneuver was possible given that most nanosatellites carry little to no propellant onboard.

\subsubsection{Results}

The results found for a simple orbital transfer from the point of minimum orbital energy in Cassini's "ball of yarn" trajectory to one of lower energy can be seen in Figure 9 and numerically Table 4.
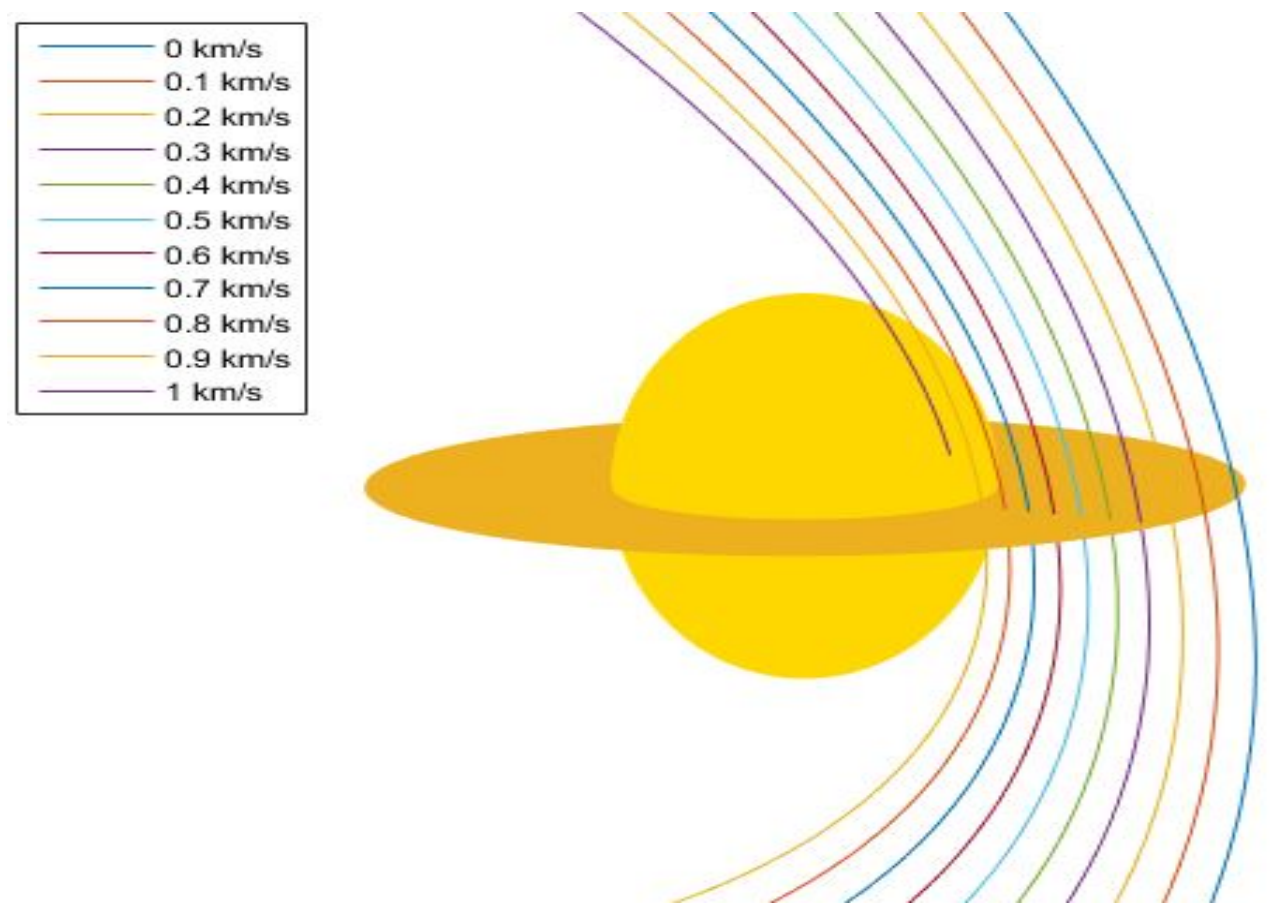

Figure 9. Saturn Ring Impactor 
Table 4. Saturn Ring Impactor Results [19]

\begin{tabular}{|c|c|c|}
\hline$\Delta \mathrm{V}(\mathrm{km} / \mathrm{s})$ & $\begin{array}{c}\text { Distance from Saturn's } \\
\text { Center at Equator }(\mathrm{km})\end{array}$ & Impact Location \\
\hline 0 & 162825.4 & No Impact \\
\hline 0.1 & 149254.9 & No Impact \\
\hline 0.2 & 136450.7 & A Ring \\
\hline 0.3 & 124378.4 & A Ring \\
\hline 0.4 & 113006.2 & B Ring \\
\hline 0.5 & 102305 & B Ring \\
\hline 0.6 & 92248 & B Ring \\
\hline 0.7 & 82810.44 & C Ring \\
\hline 0.8 & 73969.69 & D Ring \\
\hline 0.9 & 65704.93 & No Impact \\
\hline 1 & 57998.96 & Saturn \\
\hline
\end{tabular}

The results from this analysis show that for a relatively small range of Delta $\mathrm{V}, 0$ to 1 $\mathrm{km} / \mathrm{s}$, a daughter spacecraft could have impacted any location within Saturn's ring structure or multiple different areas using a series of smaller maneuvers. This would not be possible with Cassini itself due to the incurred risk from passing through the denser parts of the ring structure, however, a disposable nanosatellite could be designed for this sole purpose and would not jeopardize the main mission even if it failed.

\subsubsection{Other Options}

There are a couple of other methods for impacting the rings with a daughter probe. The first one falls in the interplanetary flyby category where a daughter spacecraft is released well outside Saturn's gravitational sphere of influence with a trajectory that allows for a flyby of Saturn with the radius of periapse at the desired altitude for the rings. This is a feasible method and would likely involve less Delta V to impact some of the lower altitudes and allows for both perpendicular and parallel trajectories through the rings. The negatives of the interplanetary flyby method are that the daughter spacecraft would be travelling at a significantly higher relative velocity of around $28 \mathrm{~km} / \mathrm{s}$. This number was found by using the average relative distance and velocity of gravity assists exiting Saturn's gravitational sphere of influence in conjunction with the average altitude of 
Saturn's rings and conservation of orbital energy seen in Equation 1. Specific Orbital Energy. This relatively large relative velocity would likely result in lower fidelity scientific data as well as a maximum of one data point since the trajectory would be a hyperbola at those speeds.

The second potential option that would reduce the relative velocity of impact would be deploying the daughter spacecraft as Cassini enters its impactor trajectory. If deployed at the correct time the daughter probe would not require any propulsion. This Extended-Extended Mission (XXM) to impact Saturn directly was decided upon after Cassini had reached Saturn so a custom daughter satellite would not be on board, however, due to their relative simplicity and modularity any impactor type nanosatellite onboard could perform the mission nominally [16]. Since the XXM has not occurred yet, the SPICE data on its trajectory is not available for analysis.

There are also a few possibilities that involve more complex trajectories. These include using a gravity assist of one of the moons to put the daughter spacecraft on the correct trajectory or using aerobraking on Titan or when entering the Saturnian system to lower the relative velocity of impact. These methods are both possibilities when considering a specific mission, but involve highly accurate attitude determination and control as well as other design considerations like heat shields that may drive the design of the nanosatellite.

\subsection{Saturn's Moons}

Though Cassini gathered a lot of data on the moons of Saturn there are still a lot of unknowns that could be solved by disposable nanosatellite probes. The moons of Saturn still have many unanswered questions. One of the most important aspects of the Cassini mission was the study of the numerous moons in the Saturnian system, but as seen in Figure 6 at the beginning of this chapter Cassini's complex trajectory only managed to gather under two hundred data points on Titan and significantly less on all of the other moons. Ideally each moon would have hundreds of data points at various seasons and a variety of longitudes, latitudes and elevations including some data points at the surface. Many of these possibilities will be explored in this section using patched conics to address the possibility of individual planetary gravity assists for the daughter spacecraft. 


\subsubsection{Setup}

Before determining potential lunar orbit trajectories, the gravitational spheres of influence of each moon must be established. The mass, distance from the center of Saturn, and surface diameter were used for Saturn and every moon in this analysis. The numbers can again be found in Table 2. For each body the mass (m) was considered to be a point mass at the center and using Equation 2. Acceleration of Gravity, an acceleration ( $\mathrm{g}$ ) was found as a function of distance from Saturn (r).

\section{Equation 2. Acceleration of Gravity$$
g=\frac{G m}{r^{2}}
$$

In Equation 2. Acceleration of Gravity, $\mathrm{G}$ is the gravitational constant at $6.6742 \mathrm{e}-20$ $\mathrm{km}^{3} / \mathrm{s}^{2} \mathrm{~kg}$. An obvious singularity occurs when the distance from the mass (r) is 0 . This is addressed by forcing the acceleration due to gravity to be constant throughout the interior of the body (i.e. $\mathrm{g}_{\text {center }}=\mathrm{g}_{\text {surface }}$ ). Each of these functions was then translated along the $\mathrm{x}$-axis using the body's average distance from the center of Saturn resulting in the image seen in Figure 10 below.

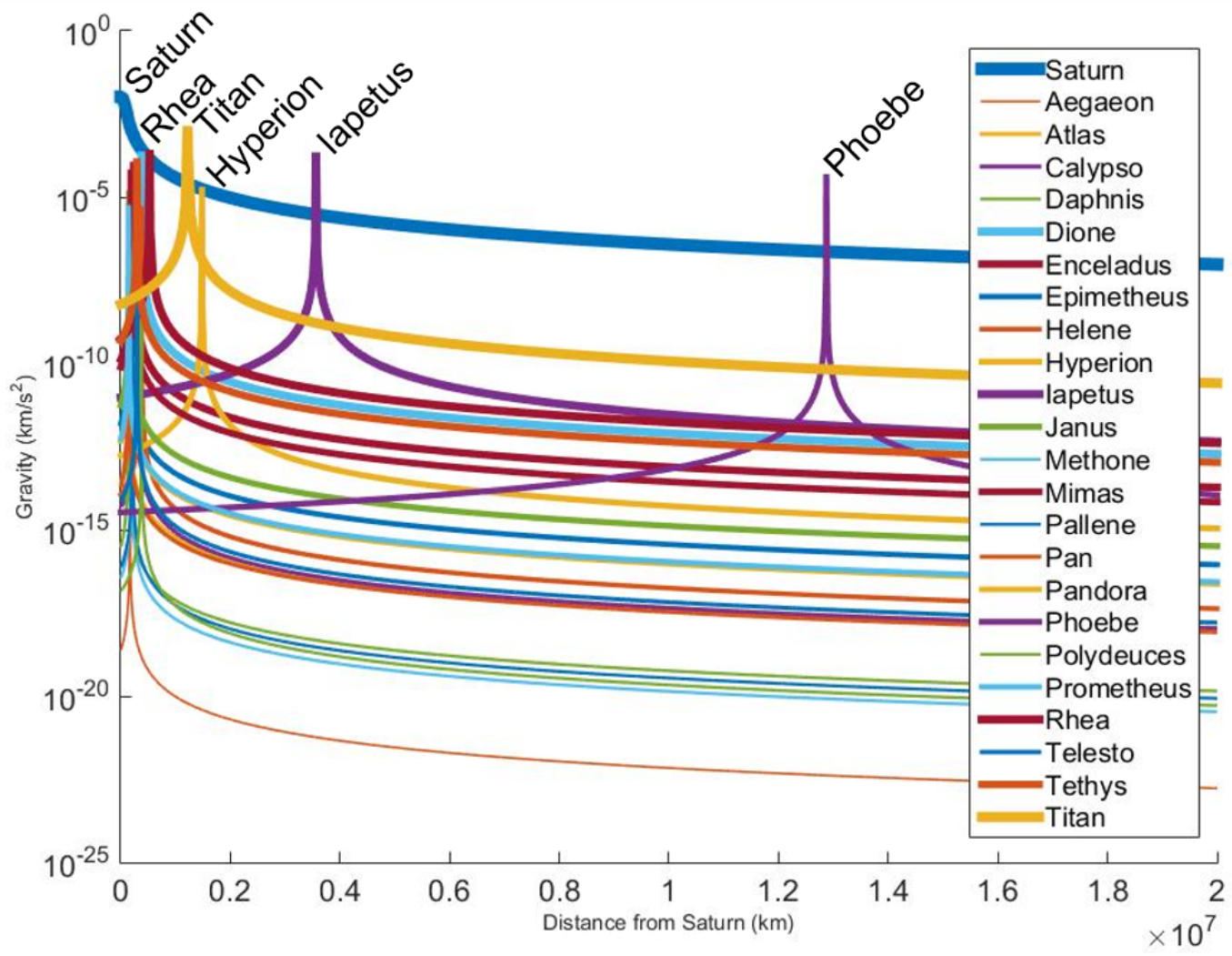

Figure 10. Gravity of the Saturnian System 
The labeled peaks identify the 6 bodies, Saturn and five of its moons, with prominent gravitational spheres of influence. For the five moon cases, a diameter of their gravitational sphere of influence in kilometers was determined through the two intersection points with Saturn's gravitational sphere of influence. The escape velocity at the surface, in kilometers per second, was also determined prior to the orbiter process using Equation 3 and the radius of the moon (r).

\section{Equation 3. Escape Velocity$$
V_{e s c}=\sqrt{\frac{2 G m}{r}}
$$

The diameter of the gravitational spheres of influence for each moon as well as their escape velocities at the surface of each moon can be seen in Table 5 below.

Table 5. Significant Gravity Wells in the Saturnian System

\begin{tabular}{|c|c|c|}
\hline Moon & SOI Diameter $(\mathrm{km})$ & $\begin{array}{c}\text { Escape Velocity at the } \\
\text { Surface }(\mathrm{km} / \mathrm{s})\end{array}$ \\
\hline Hyperion & 100 & 0.0745 \\
\hline Iapetus & 13,000 & 0.5718 \\
\hline Phoebe & 3,000 & 0.1019 \\
\hline Rhea & 2,180 & 0.6341 \\
\hline Titan & 37,700 & 2.6454 \\
\hline
\end{tabular}

\subsubsection{Impactors}

The first and probably the most practical use for nanosatellite probes would be the impactor. Impactors allow for the gathering of in situ data that cannot otherwise be measured with modern technology from a distance, but typically result in the end of life of the vehicle. One example of an impactor was the Lunar CRater Observation and Sensing Satellite (LCROSS) which impacted the Moon on October 9, 2009 to determine if the moon contained water ice in a permanently shadowed crater [20].

\subsubsection{Process}

The process for finding potential impact cases starts by looking at a specific flyby for a single moon at a certain time, which can be read from the complete version of Table 3 . Figure 11 
below is an example on one such discrete case where the target moon is Titan and the certain time is July 2, 2001 at 9:29 Spacecraft Event Time (SCET).

\section{Titan}

\section{4-Jul-02 9:29}

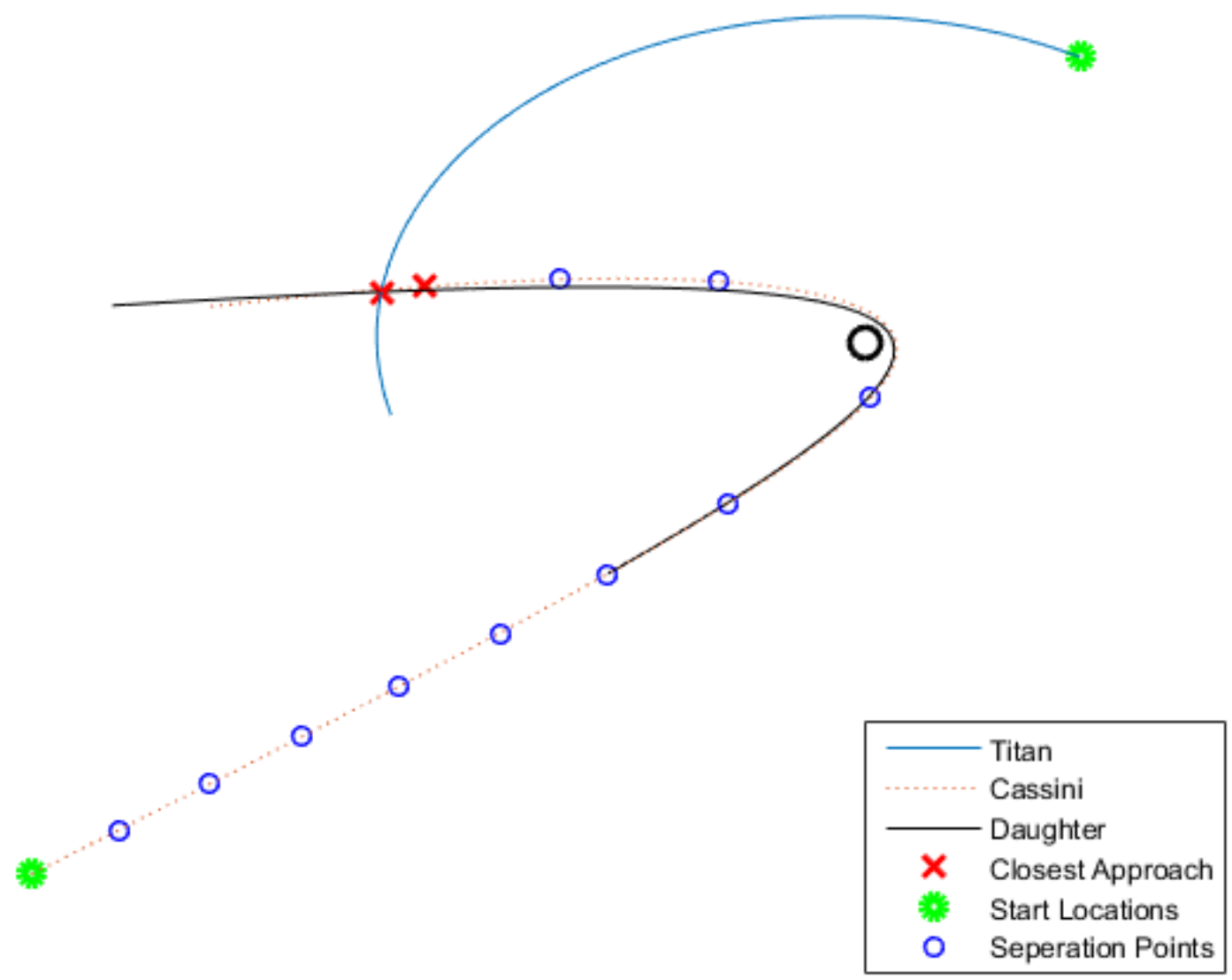

Figure 11. Moon Impactor Example

Since these flybys do not have a specific start and end time, arbitrary start and end times were selected for the analysis. The analysis utilizes a 25 day timespan with 20 days before the approximate closest approach and 5 days after the specified time. This scope was found through experimentation to best encapsulate the range of possibilities in each case without requiring significant computational power. The reason for the asymmetry was that the 20 days allows for a well-spaced set of launch points and the 5 days after ensures that the exact point of closest approach was within the timespan for each case. The timespan could be extended beyond 20 days, however there are diminishing returns and the results would not radically differ from the ones seen 
in this analysis. This was in part due to the orbital period of Cassini as well as the fidelity of the orbital propagation employed.

This timespan was then used in conjunction with the trajectories for Cassini and the target moon at that time to find the exact time of closest approach for Cassini, as seen in Figure 11 as two red X's. This exact time of closest approach became the upper bound for potential launch points for an impactor probe, since any point after would require significant Delta $\mathrm{V}$ to reverse the velocity vector and achieve impact. The lower bound for potential launch points is the beginning of the timespan. Also, the position of the target moon that corresponds with the exact time of closest approach is selected to be the target for the Lambert's solver for each variation. The Lambert's solver used was the Izzo-Gooding Lambert's solver available in MatLab.

The initial deployment window is broken into a set of initial separation points, where a daughter spacecraft could hypothetically be deployed from and then perform an instantaneous maneuver. This maneuver is selected to place the daughter spacecraft on an impact trajectory with the target moon using the Lambert's solver. For this analysis there are 10 initial points of possibility, which can be seen as blue circles in Figure 11. These 10 cases, when propagated, serve to anchor trends and patterns to guide high-level selection of potential missions.

Each of these separation points in blue is used as an initial position for a Lambert's solver with the time and final position already determined by the exact closest approach of Cassini to the target moon. Using other target positions and corresponding flight times could introduce new trajectories for impact, but the flight times and Delta $\mathrm{V}$ requirements would likely be less desirable. Through a more in depth study of a specific moon and flyby it would be possible to explore these options, however exploring all of Cassini's lunar flybys in this fashion is outside the scope of this study.

The resulting velocities from the Lambert's solutions were used in conjunction with the velocities of Cassini and the target moon to find the initial Delta V of the maneuver and the relative impact velocity respectively. The Delta $\mathrm{V}$ of the maneuver was then broken up into a unit direction vector and a magnitude. The magnitude of Delta $\mathrm{V}$ was then fractionalized into evenly spaced magnitudes. An example required Delta V of $120 \mathrm{~m} / \mathrm{s}$ would then be split up into $100 \mathrm{~m} / \mathrm{s}$, 
$90 \mathrm{~m} / \mathrm{s}, 80 \mathrm{~m} / \mathrm{s}$, and so on. For this analysis, there were 10 Delta V magnitudes for each Lambert's solution. Again, 10 discrete points was selected to get a broad understanding of the effects that a varying Delta V would have on the system. These different magnitudes were sequentially multiplied to the unit direction vector to determine the results from less ideal maneuvers. Each of the resulting states was then propagated forward in time using a variable step Runge-Kutta 4th and 5th Order Method. The variable step Runge-Kutta 4th and 5th Order Method employed was the MathWorks built in propagator ode 45.

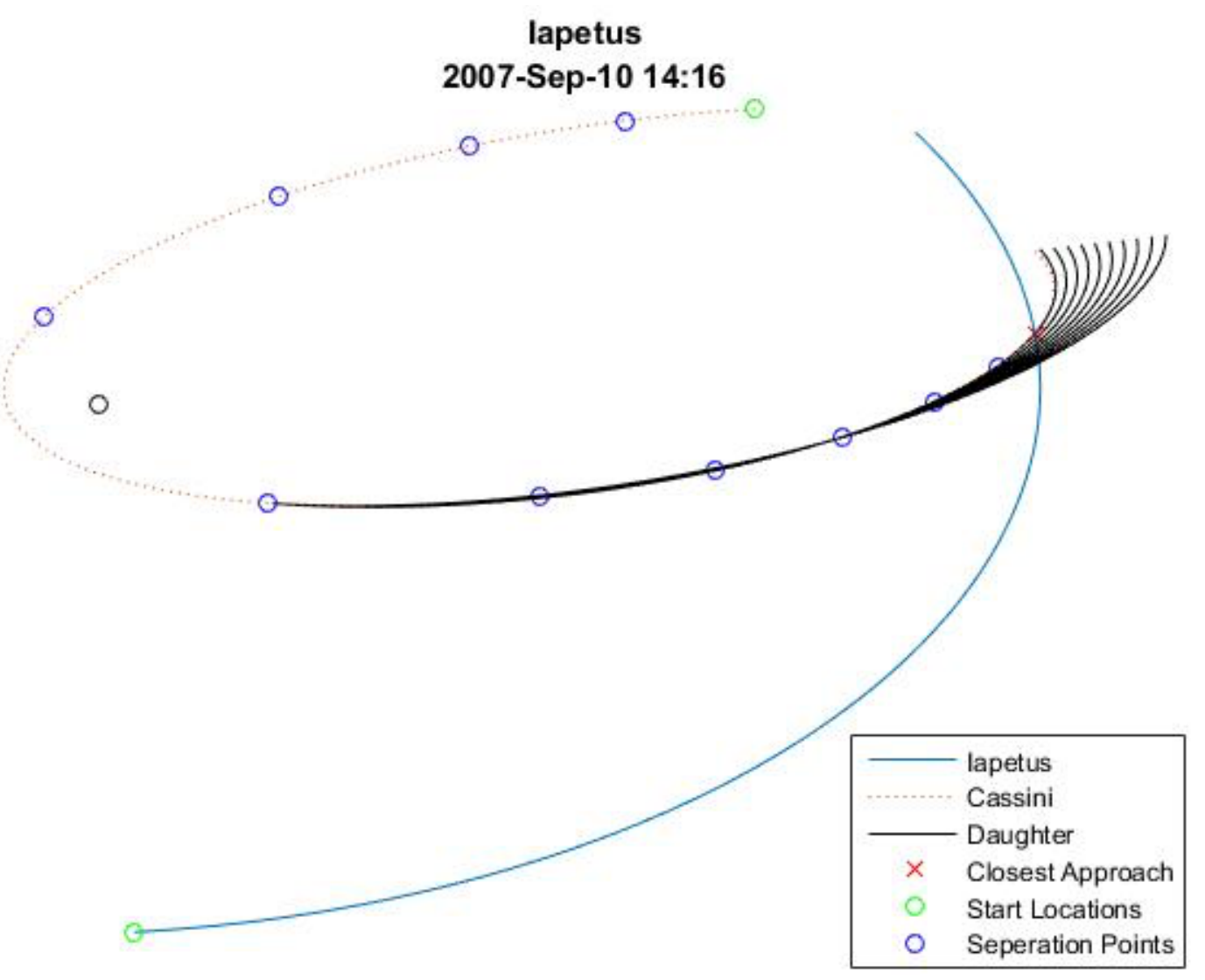

Figure 12. Moon Impactor Example with Variable Delta V

The resulting states from the various deployment times as well as the varying magnitudes of Delta $\mathrm{V}$ were recorded in a database of outputs. An excerpt from this database can be seen in Table 6 below. This table also corresponds with the trajectories in Figure 12. For each of these possibilities, first the name of the target moon and the approximate date and time of closest approach were recorded. The release time, in days prior to Cassini's exact closest approach were 
recorded in the third column. The magnitude of Delta $\mathrm{V}$ for the initial maneuver in kilometers per second was recorded in the fourth column.

Table 6. Excerpt of Moon Trajectory Data

\begin{tabular}{|c|c|c|c|c|c|c|}
\hline Target & Date & $\begin{array}{c}\text { Release } \\
(\text { days })\end{array}$ & $\begin{array}{c}\text { Initial } \\
\Delta \mathrm{V} \\
(\mathrm{km} / \mathrm{s})\end{array}$ & $\begin{array}{c}\text { Closest } \\
\text { Approach } \\
(\mathrm{km})\end{array}$ & $\begin{array}{c}\text { Final } \mathrm{V}_{\text {rel }} \\
(\mathrm{km} / \mathrm{s})\end{array}$ & $\begin{array}{c}\text { Min. } \Delta \mathrm{V} \\
\text { to Orbit } \\
(\mathrm{km} / \mathrm{s})\end{array}$ \\
\hline Iapetus & $\begin{array}{c}2007-\mathrm{Sep}-10 \\
14: 16\end{array}$ & 10.86 & 0.054 & 340,478 & 2.455 & $\begin{array}{c}\text { Outside } \\
\text { SOI }\end{array}$ \\
\hline Iapetus & $\begin{array}{c}2007-\mathrm{Sep}-10 \\
14: 16\end{array}$ & 10.86 & 0.108 & 301,621 & 2.438 & $\begin{array}{c}\text { Outside } \\
\text { SOI }\end{array}$ \\
\hline Iapetus & $\begin{array}{c}2007-\text { Sep-10 } \\
14: 16\end{array}$ & 10.86 & 0.163 & 262,223 & 2.421 & $\begin{array}{c}\text { Outside } \\
\text { SOI }\end{array}$ \\
\hline Iapetus & $\begin{array}{c}2007-S e p-10 \\
14: 16\end{array}$ & 10.86 & 0.217 & 223,756 & 2.406 & $\begin{array}{c}\text { Outside } \\
\text { SOI }\end{array}$ \\
\hline Iapetus & $\begin{array}{c}2007-S e p-10 \\
14: 16\end{array}$ & 10.86 & 0.271 & 185,566 & 2.390 & $\begin{array}{c}\text { Outside } \\
\text { SOI }\end{array}$ \\
\hline Iapetus & $\begin{array}{c}2007-S e p-10 \\
14: 16\end{array}$ & 10.86 & 0.325 & 147,647 & 2.376 & $\begin{array}{c}\text { Outside } \\
\text { SOI }\end{array}$ \\
\hline Iapetus & $\begin{array}{c}2007-S e p-10 \\
14: 16\end{array}$ & 10.86 & 0.379 & 110,078 & 2.362 & $\begin{array}{c}\text { Outside } \\
\text { SOI }\end{array}$ \\
\hline Iapetus & $\begin{array}{c}2007-S e p-10 \\
14: 16\end{array}$ & 10.86 & 0.433 & 72,892 & 2.349 & $\begin{array}{c}\text { Outside } \\
\text { SOI }\end{array}$ \\
\hline Iapetus & $\begin{array}{c}2007-S e p-10 \\
14: 16\end{array}$ & 10.86 & 0.488 & 35,411 & 2.336 & $\begin{array}{c}\text { Outside } \\
\text { SOI }\end{array}$ \\
\hline Iapetus & $\begin{array}{c}2007-S e p-10 \\
14: 16\end{array}$ & 10.86 & 0.542 & -735 & 2.325 & 2.295 \\
\hline
\end{tabular}

The closest approach in kilometers identifies the minimum distance between the daughter spacecraft and the target moon within the timespan specified. A negative number in the closest approach column signifies that the daughter spacecraft would impact the target moon. This can be seen in the green cell of Table 6 . The final relative velocity in kilometers per second is the 2-norm of the velocity of the daughter spacecraft subtracted by the target moon at closest approach. This final relative velocity can be used to approximate the impact velocity of the impactors or to calculate the minimum Delta $\mathrm{V}$ required to enter orbit. The final column, Minimum Delta $\mathrm{V}$ to Orbit, identifies the total amount of Delta $\mathrm{V}$ needed onboard a daughter spacecraft to enter a highly elliptical orbit. The process for achieving the last column is covered in Section 3.3.3.

\subsubsection{Unique Case}

This information can be used to form an initial plan for the trajectory of an impactor for 
any moon of interest during Cassini's tour of the Saturnian system. The processes involved can also be used for planning future mother-daughter architecture. Since planning a wide variety of custom missions goes beyond the scope of this thesis, a single unique case shall be considered as an example.

One likely case of interest would be a proof of concept, where the designer wishes to prove that a daughter nanosatellite impactor is in fact possible. This lends itself to finding the most viable mission requirements for the daughter spacecraft; requirements like small amounts of stored Delta V, minimal time exposed to the Saturnian environment prior to impact, and perhaps a low relative velocity to improve data sampling. Using the spreadsheet it is relatively simple to find a mission that satisfies these requirements. The process of finding mission parameters can be done by comparing various cases with filters and sorting or by weighting all of the variables (Delta V, exposure time, relative impact velocity, etc.) and then optimizing the result. This process would likely result in a list of desirable cases like the one in Table 7 below.

Table 7. Impactor Proof of Concept

\begin{tabular}{|c|c|c|c|c|c|c|}
\hline Target & Date & $\begin{array}{c}\text { Release } \\
(\text { days })\end{array}$ & $\begin{array}{c}\text { Initial } \\
\Delta \mathrm{V} \\
(\mathrm{km} / \mathrm{s})\end{array}$ & $\begin{array}{c}\text { Closest } \\
\text { Approach } \\
(\mathrm{km})\end{array}$ & $\begin{array}{c}\text { Final } \mathrm{V}_{\text {rel }} \\
(\mathrm{km} / \mathrm{s})\end{array}$ & $\begin{array}{c}\text { Min. } \Delta \mathrm{V} \\
\text { to Orbit } \\
(\mathrm{km} / \mathrm{s})\end{array}$ \\
\hline Titan & $\begin{array}{c}2008-\text { Aug-15 } \\
20: 47\end{array}$ & 2.35 & 0.001 & -1673 & 5.879 & 3.235 \\
\hline Titan & $\begin{array}{c}2007-\text { May-28 } \\
18: 52\end{array}$ & 2.93 & 0.001 & -325 & 5.824 & 3.180 \\
\hline Titan & $\begin{array}{c}2007-\text { May-28 } \\
18: 52\end{array}$ & 3.30 & 0.001 & -316 & 5.823 & 3.179 \\
\hline Titan & $\begin{array}{c}2006-\text { Feb-27 } \\
8: 25\end{array}$ & 3.55 & 0.001 & -401 & 5.526 & 2.882 \\
\hline Titan & $\begin{array}{c}2006-\text { Apr-30 } \\
20: 58\end{array}$ & 3.55 & 0.001 & -431 & 5.507 & 2.863 \\
\hline Titan & $\begin{array}{c}2006-J u l-02 \\
9: 21\end{array}$ & 3.55 & 0.001 & -564 & 5.502 & 2.857 \\
\hline Titan & $\begin{array}{c}2011-J u n-20 \\
18: 32\end{array}$ & 3.55 & 0.001 & -341 & 5.509 & 2.864 \\
\hline Titan & $\begin{array}{c}2007-\text { May-28 } \\
18: 52\end{array}$ & 3.68 & 0.001 & -299 & 5.822 & 3.178 \\
\hline Titan & $\begin{array}{c}2007-\text { May-28 } \\
18: 52\end{array}$ & 4.05 & 0.001 & -271 & 5.822 & 3.178 \\
\hline Titan & $\begin{array}{c}2006-\text { Feb-27 } \\
8: 25\end{array}$ & 5.38 & 0.001 & -219 & 5.525 & 2.881 \\
\hline
\end{tabular}


The top result in Table 7 for Titan on August 15, 2008 can be used to begin the design process for an impactor. The daughter spacecraft will need to possess enough power to last 2.35 days after deployment, likely also accounting for the 10 years and 305 days of battery selfdischarging. If the self-discharge rate of the battery is an issue the selection of a new case can also consider the deployment date. For this specific case the use of onboard propulsion for the daughter spacecraft might be unnecessary since most nanosatellite deployers can already impart velocities on the order of single meters per second [21].

The tolerances for Delta V of the daughter spacecraft and the time of deployment need to be evaluated to create requirements for the windows available for impact. A better understanding can be established by observing the other cases for Titan on August 15, 2008, these can be seen below in Figure 13.

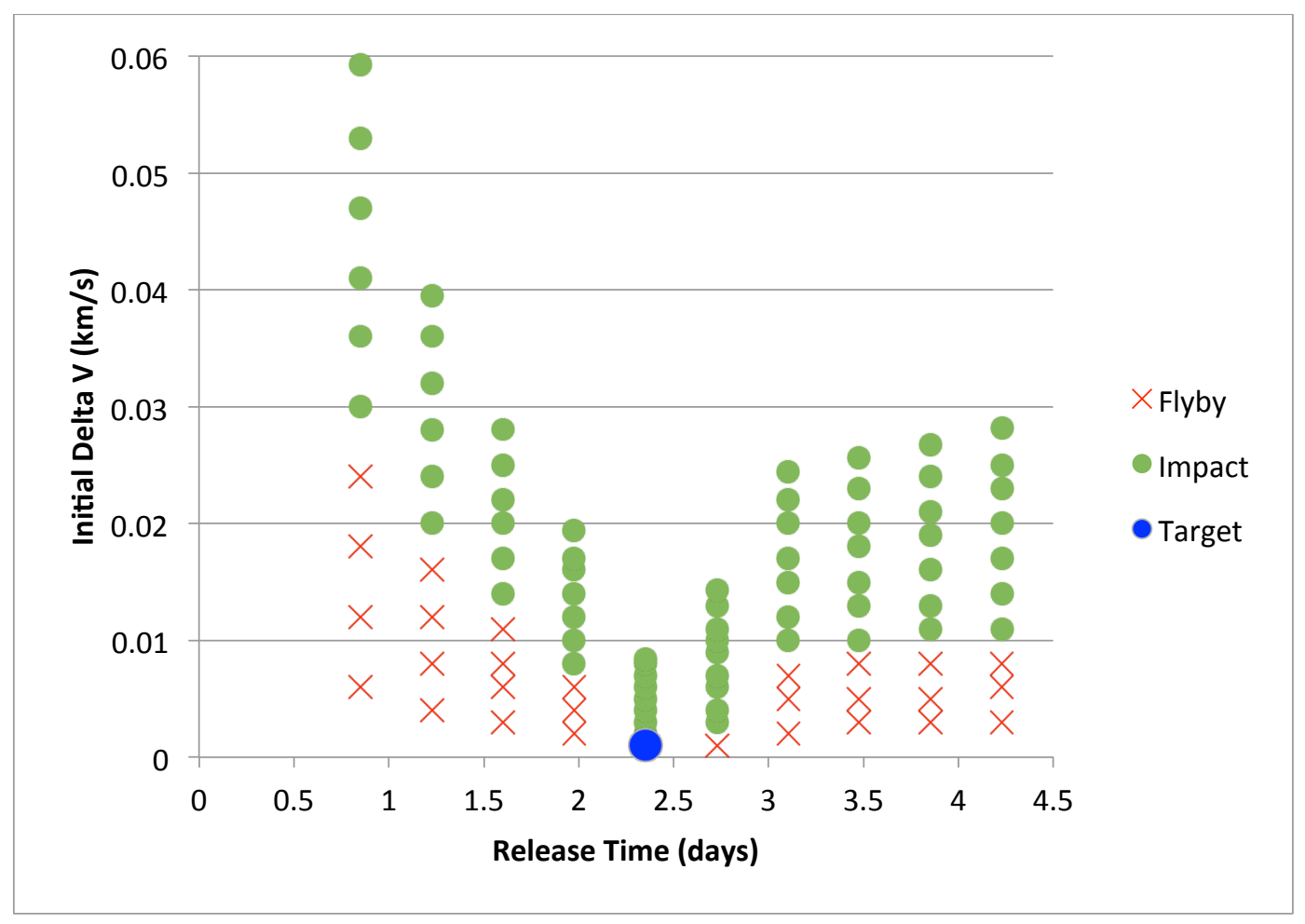

Figure 13. Release Time and Initial Delta V Envelope

Figure 13 shows the target deployment (Target) for the proof of concept as well as acceptable (Impact) and unacceptable (Flyby) alternatives due to inaccuracies in the release time and initial Delta V. The upper most Impact point identifies a trajectory through the core of the 
moon. If the accuracy of the release time is a point of concern the Delta $\mathrm{V}$ of deployment can be increased to improve the tolerances, but this is unlikely since the release time is on the order of days rather than milliseconds which is a frequency that most computers can operate at. The unexpected increase of Delta $\mathrm{V}$ at deployment also does not appear to be an area for concern since the deployment Delta V needs to increase by an order of magnitude in order to overshoot Titan. The decrease of the deployment velocity would be an issue to consider in the design phase, but overall the tolerances for this proof of concept deployment is relatively loose.

Another tolerance to be considered would be the accuracy of the deployment Delta V vector. This deployment vector relies on the accuracy of the mother spacecraft's attitude determination and control system as well as the accuracy of the daughter spacecraft deployer. The mother spacecraft's attitude determination and control system is usually driven by high precision ranged instruments like on Cassini, meaning that the accuracy is pretty high relative to nanosatellite standards. The nanosatellite deployer on the other hand is not typically designed for precision. Tests would have to be performed to induce the accuracy of deployment and then apply it to simulations to determine whether the performance is acceptable. Spinning up a momentum wheel within the daughter spacecraft prior to deployment might help this accuracy. Based off the results found in Figure 13 it can be deduced that the accuracy of the deployment vector will not be a driver in this proof of concept impactor design.

From the high level analysis, it is possible to begin the engineering process involved in designing any particular case, and the fidelity of the trajectory requirements can be refined along side the design process. A set of design requirements for a low thrust impactor of Enceladus, a low angle impact through Titan's atmosphere, or any other impactor desired can be produced by a similar process.

\subsubsection{Totals}

For comprehension, the data of all impactor cases has been summarized in a few figures, which can be seen below. 


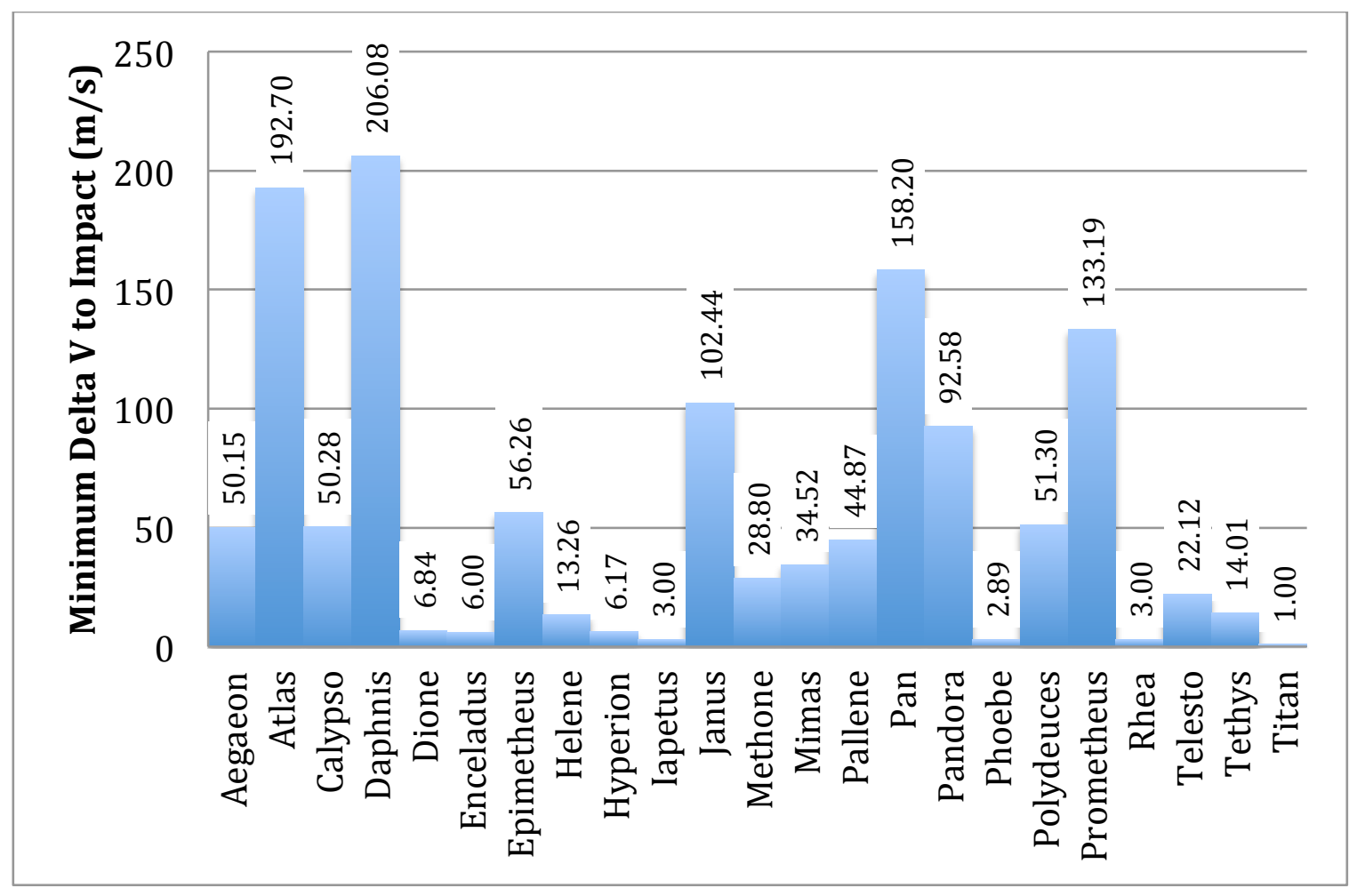

Figure 14. Minimum Delta V to Impact Saturn's Moons

Figure 14 shows the minimum recorded Delta V required to impact each of Saturn's moons. Again this analysis did not consider cases that orbited Saturn prior to impact or cases that allowed for variance in the time of impact, but the results are a good starting value when considering the implications of impacting one of the moons. Hyperion, Iapetus, Phoebe, Rhea, and Titan are among the moons with the lowest required Delta V, which makes sense since these are the five moons with their own gravitational sphere of influence that trump that of Saturn's gravity. 9 moons are attainable with only 20 meters per second of Delta V, 13 moons can be impacted with less than $50 \mathrm{~m} / \mathrm{s}$ of Delta $\mathrm{V}$, and an additional 4 moons require around $50 \mathrm{~m} / \mathrm{s}$ (Aegaeon, Calypso, Epimetheus, and Polydeuces). Atlas, Daphnis, Pan, and Prometheus would require a significant amount of propulsion to impact, however these magnitudes are still attainable. Current nanosatellite propulsion can reliably achieve $410 \mathrm{~m} / \mathrm{s}$ of Delta $\mathrm{V}$ in less than a 1,000 cubic centimeters [22]. 
Figure 15 and Figure 16 below show the number of potential impact cases as a function of how much Delta V the daughter spacecraft has onboard. Figure 17 shows the same data as a percentage of the total amount of cases available.

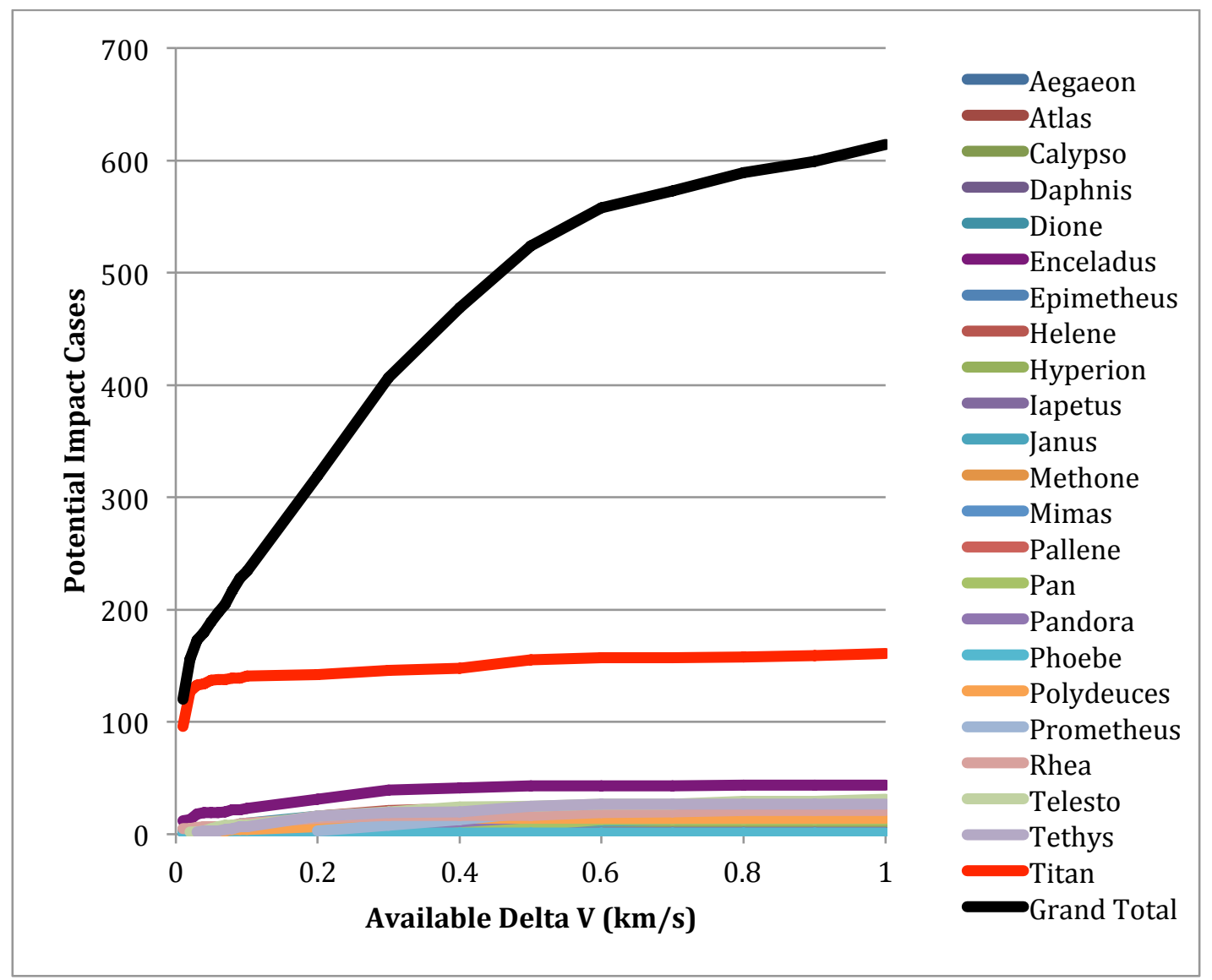

Figure 15. Potential Impact Cases (0-700 Cases)

Out of Cassini's 709 moon flyby cases considered $614(87 \%)$ would allow for an impactor with $1 \mathrm{~km} / \mathrm{s}$ Delta V. At $.1 \mathrm{~km} / \mathrm{s}$ there are 234 (33\%) potential impact cases. Titan and Enceladus (Red and Violet) each received numerous flybys, which is why in Figure 15 they have more potential impact cases. Figure 16 displays the same information, but with a smaller vertical range to better display the less popular moons. Phoebe, the least popular moon due to its relatively large semi major axis only had one considered flyby, but the relative position and velocity of the pass were small in magnitude allowing for a smaller deployment maneuver needed for impact. 


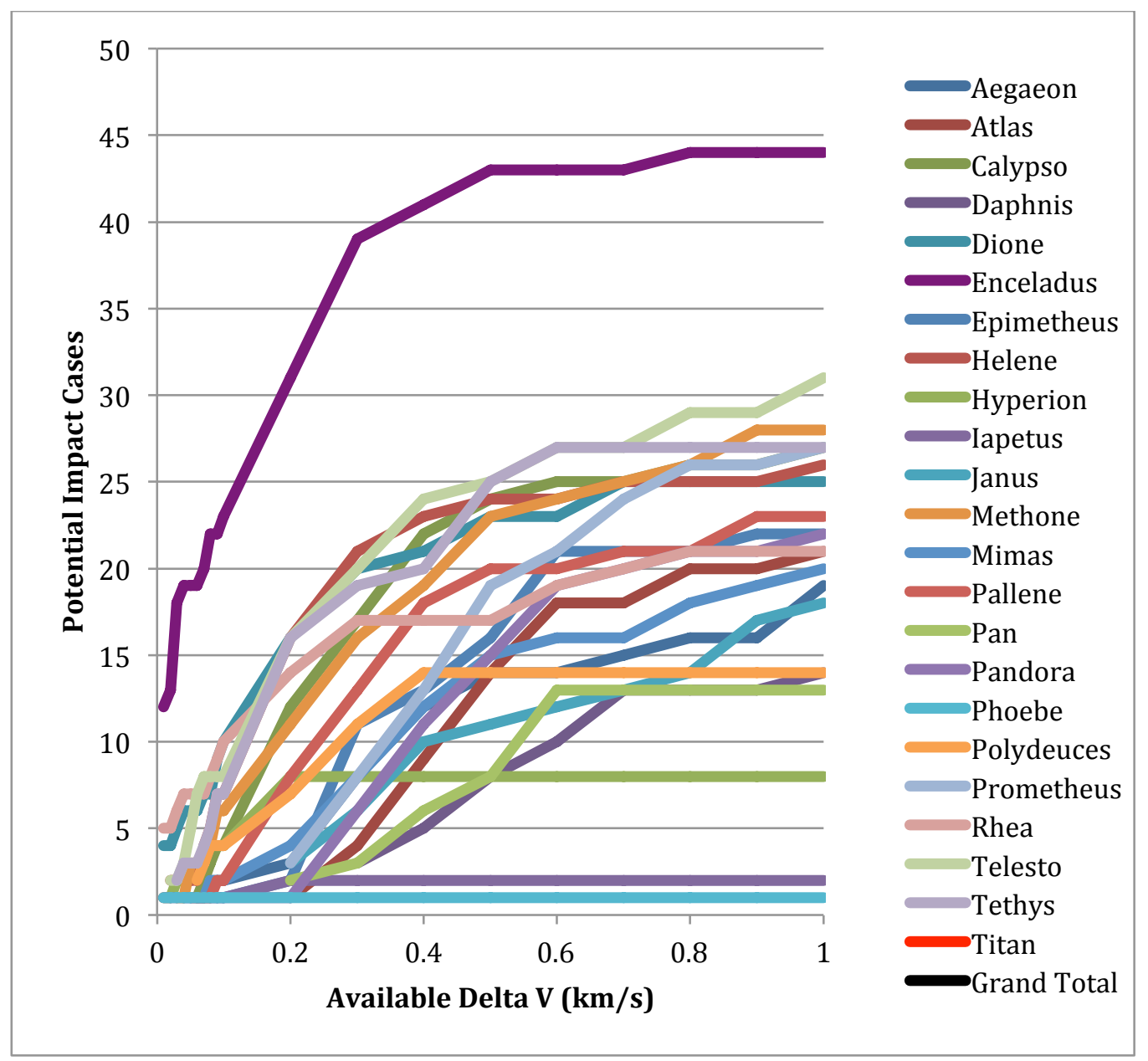

Figure 16. Potential Impact Cases (0-50 Cases) 


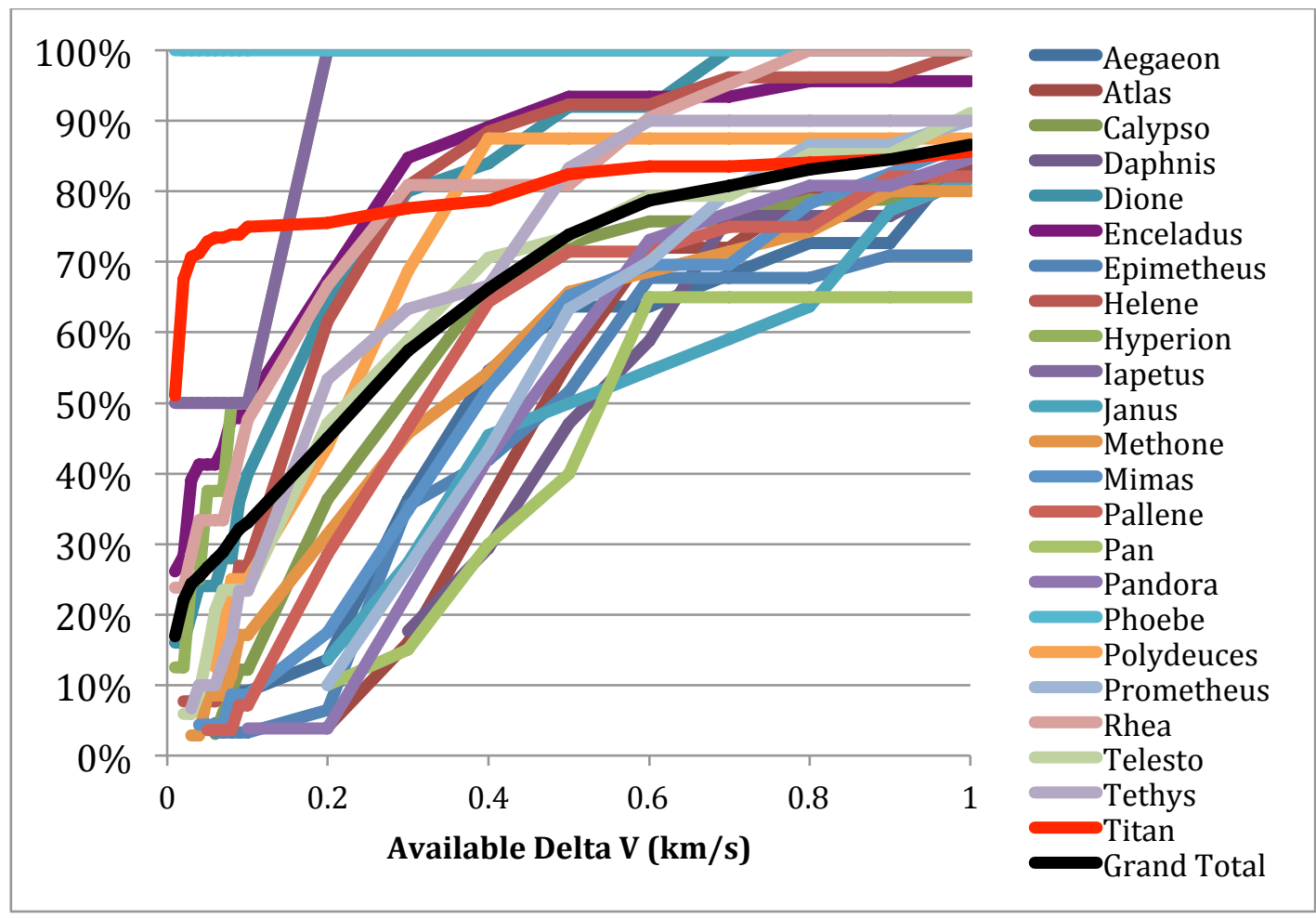

Figure 17. Potential Impact Cases (Percentage)

While the results for each moon, seen in Figure 17, varies there typically exists a drop off point. For Titan, in red, that drop off point appears to be $.1 \mathrm{~km} / \mathrm{s}$ where the percentage of available cases drops off significantly. This information can be useful in the design of a more flexible daughter mission where the impactor location is not decided upon until after launch. In this case picking a Delta $\mathrm{V}$ budget that encompasses a certain percentage of the flybys would likely be the logical course of action; this could be the grand total or more focused on a particular moon depending on the scientific goals.

This analysis encompasses all possible simple trajectories. The use of more complex trajectories for nanosatellites is possible and may allow for smaller numbers in terms of Delta $\mathrm{V}$ to Impact and Potential Impact Cases. One example would be the deployment of a daughter spacecraft prior to entering the Saturnian System that would maneuver its trajectory to impact the harder to reach moons of Saturn at a much higher relative velocity and with much less margin for error. Most of these complex cases inherently result in much lower tolerances, higher exposure to the Saturn's environments, and as a result less resources allocated to the Payload and its primary 
directive. Another potential issue for impactor spacecraft is planetary protection, meaning that the impactors need to meet certain standards prior to launch to ensure they do not contaminate the moon. This sterilization will likely be easier to achieve in nanosatellites due to their relative simplicity and small size.

\subsubsection{Orbiters}

Another desirable mission type for a simple nanosatellite, the orbiter allows for numerous data points to be gathered all around a celestial body. Orbiters also spend the most time operating near the point of interest since it neither leaves nor crashes into the body, which allows for a more sustainable mission. Orbiters are the most common missions for nanosatellites to date. They have been used for everything from global imaging [23] to measuring particles in the atmosphere [24]. In interplanetary missions however unique orbit trajectories are much more difficult for nanosatellites due to the typically large Delta $\mathrm{V}$ requirements.

\subsubsection{Process}

The minimum Delta $\mathrm{V}$ to orbit in kilometers per second is an approximation for the total amount of Delta V that a daughter spacecraft would need to have in order to enter a highly elliptical orbit around the target moon. The process starts by considering the five moons with their own unique gravitational sphere of influence: Hyperion, Iapetus, Phoebe, Rhea, and Titan. Pseudo-orbits around the other 18 moons are possible using relative formation flight with a slightly elliptical orbit and the same orbital period as the moon, but due to their complexity are not considered here.

This process was performed simultaneously with the impactor and flyby analysis for efficiency, where any impactor or flyby within the moon's gravitational sphere of influence was evaluated. To find the minimum Delta $\mathrm{V}$ to enter orbit the difference was taken between the relative velocity of the daughter spacecraft and the escape velocity at the distance of its closest approach. The resulting difference in velocity must be compensated by a Delta V maneuver. The Delta $\mathrm{V}$ required to enter that initial flyby or impact trajectory was added to the number before being recorded in the last column as seen in the earlier excerpt from Table 6. Any flyby 
trajectories that did not pass through a moon's gravitational sphere of influence were not considered.

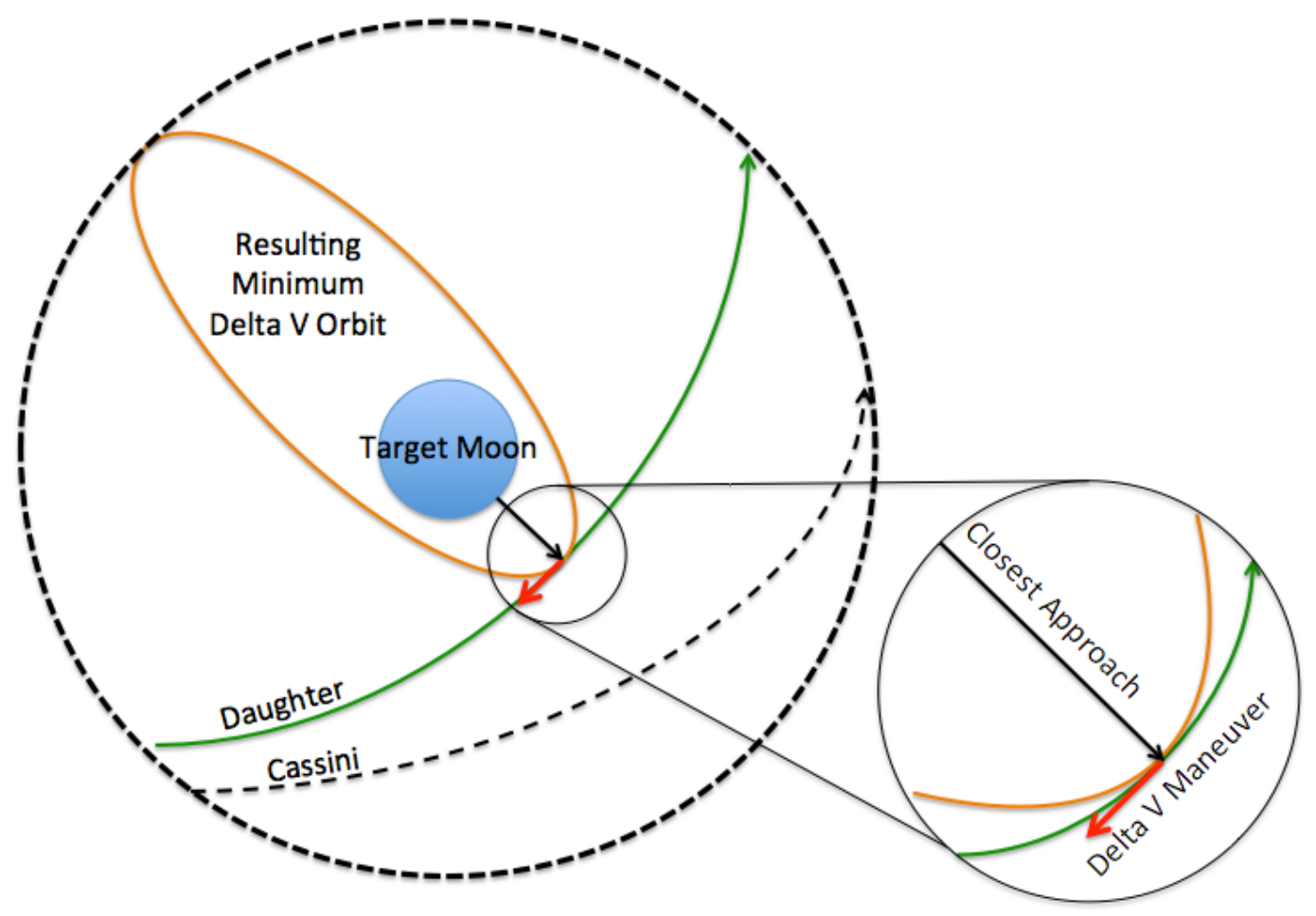

Figure 18. General Moon Orbit Solution

For cases with impact trajectories, the escape velocity at the surface seen in Table 5 was used. The Delta $\mathrm{V}$ required to impact a moon was always larger than the Delta $\mathrm{V}$ to flyby for all of the cases considered, so this process still serves as a safe estimate that can be elaborated upon once a specific case has been selected.

\subsubsection{Results}

For convenience this information has been processed and summarized in Figure 19, Figure 20, and Figure 21. 


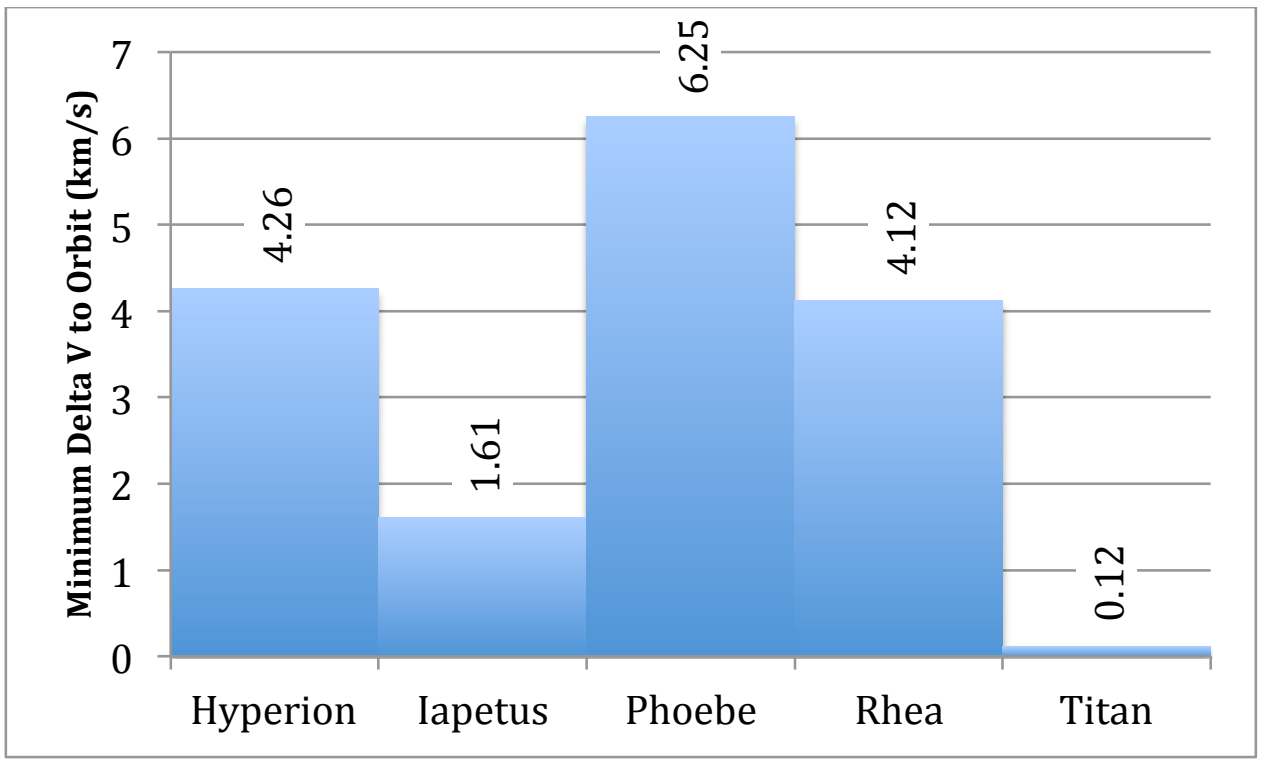

Figure 19. Minimum Delta V to Orbit Saturn's Moons

Figure 19 shows the approximate minimum required Delta $\mathrm{V}$ to enter orbit around Saturn's five moons with significant gravity. The most fuel-efficient moon to orbit with Cassini's initial trajectory is Titan, which is also Saturn's most massive moon. Phoebe and Rhea are the least massive out of the five moons and so it is logical that they would require more Delta $\mathrm{V}$ to slow down enough to enter orbit. Another major factor to these results is Cassini's trajectory, which passed by Titan 188 times while only doing one flyby of Phoebe [9]. The large inequality in flybys reasonably leads to more favorable trajectories for a simple orbit transfer around Titan, which is clear to see in Figure 19.

This inequality of cases is even clearer when looking at Figure 20 where Phoebe is not even on the chart since its Delta V requirement exceeds the range. Titan and the grand total are almost synonymous while the other three moons have only 6 possible cases available at $5 \mathrm{~km} / \mathrm{s}$. At $1 \mathrm{~km} / \mathrm{s}$ the only feasible target is one of four Titan flybys. Granted, these numbers are rough estimates, but even the magnitudes demonstrate that getting daughter spacecraft into orbit around any moon other than Titan using a simple trajectory is will require propellant to be a driving factor due to the large Delta V. 


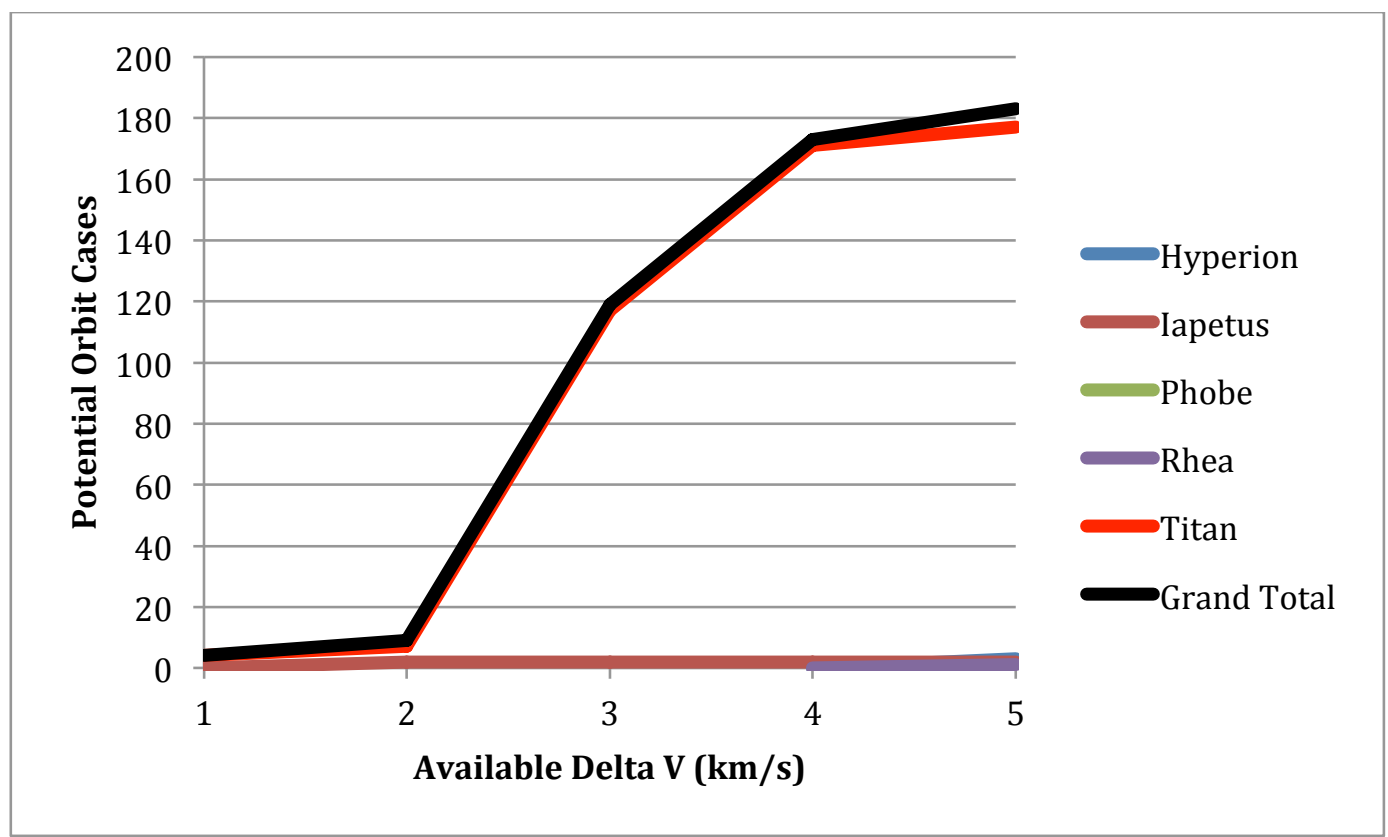

Figure 20. Potential Orbit Cases (0-200 Cases)

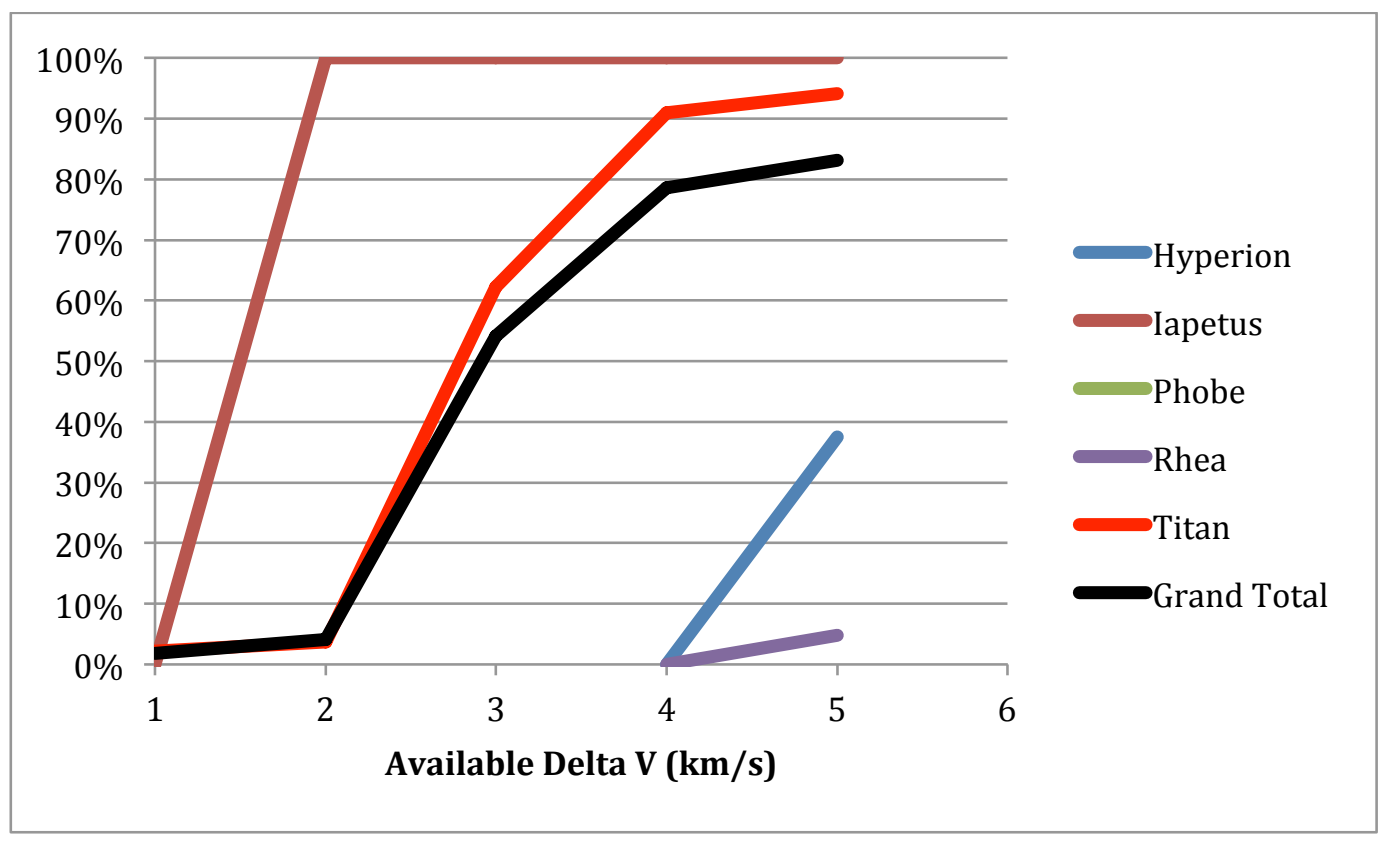

Figure 21. Potential Orbit Cases (Percentage)

Figure 21 above shows the percentage of cases that orbit is possible for a given amount of available Delta V in kilometers per second. 
In order to achieve a more circular orbit around these moons even more Delta $\mathrm{V}$ is required with the exception of using aerobraking around Titan. Orbiting the other moons with less prominent gravity fields will require the use of formation flying and relative motion, which is manageable but requires more advanced maneuvering of a nanosatellite.

\subsubsection{Flybys}

Probably the simplest trajectory for a daughter spacecraft, the flyby of Saturn's various moons can occur by simply separating from the mothership. These flybys can be used to get a closer look at the various moons. By using more Delta $\mathrm{V}$ than the impactors it is also possible to collect data from the other side of a moon than Cassini. Most of the flyby trajectories are analyzed at the same time as the impactor study since they both only utilize a single separation maneuver and overall process. The flyby trajectories are the most feasible of all of the cases studied and because of that the possibilities are infinite. Depending on the magnitude, direction, and timing of the deployment maneuver flybys of all of the various moves can be achieved. For close approach flybys the Delta V required is only slightly less than those seen in the impactor study. If Delta V is the limiting factor in the daughter spacecraft, then a more distant flyby might be the best option but the distinctiveness of the data would be limited. While flybys like the ones described are definitely feasible and relatively simple, the data potential makes the flyby less desirable than the other possible mission types explored.

\subsection{Summary}

The three major types of trajectories impactors, orbiters, and flybys have all been explored as possibilities for a Cassini based Mother-Daughter architecture. For all cases with the Saturnian system, flybys were found to be possible even with no Delta V, since Cassini's trajectory was already designed to do flybys. With impactors, the amount of Delta V and release time requirements were slightly more stringent. The opportunity for impactors can be seen with respect to the moon and amount of Delta $\mathrm{V}$ in Table 8 as the light blue fill. The ability to attain a highly elliptical orbit around the five moons of Saturn with their own significant gravity well can be seen in Table 8 as the dark blue fill with the green circle. Phoebe could not be orbited with 
under $5 \mathrm{~km} / \mathrm{s}$ of Delta $\mathrm{V}$ and as seen earlier most of the orbit possibilities were around Titan. At the bottom of Table 8 are the total amount of possible impactor and orbiter cases for each Delta V.

Table 8. Saturn's Moon Exploration Possibilities

Moon
Aegaeon
Atlas
Calypso
Daphnis
Dione
Enceladus
Epimetheus
Helene
Hyperion
lapetus
Janus
Methone
Mimas
Pallene
Pan
Pandora
Phoebe
Polydeuces
Prometheus
Rhea
Telesto
Tethys
Titan
Total Impact Cases
Total Orbit Cases

\begin{tabular}{|c|c|c|c|c|c|c|c|c|c|c|c|c|c|c|}
\hline $5 \mathrm{~km} / \mathrm{s}$ & $4 \mathrm{~km} / \mathrm{s}$ & $3 \mathrm{~km} / \mathrm{s}$ & $2 \mathrm{~km} / \mathrm{s}$ & $1 \mathrm{~km} / \mathrm{s}$ & $500 \mathrm{~m} / \mathrm{s}$ & $400 \mathrm{~m} / \mathrm{s}$ & $300 \mathrm{~m} / \mathrm{s}$ & $200 \mathrm{~m} / \mathrm{s}$ & $100 \mathrm{~m} / \mathrm{s}$ & $50 \mathrm{~m} / \mathrm{s}$ & $40 \mathrm{~m} / \mathrm{s}$ & $30 \mathrm{~m} / \mathrm{s}$ & $20 \mathrm{~m} / \mathrm{s}$ & $10 \mathrm{~m} / \mathrm{s}$ \\
\hline & & & & & & & & & & & & & & \\
\hline & & & & & & & & & & & & & & \\
\hline & & & & & & & & & & & & & & \\
\hline & & & & & & & & & & & & & & \\
\hline & & & & & & & & & & & & & & \\
\hline & & & & & & & & & & & & & & \\
\hline & & & & & & & & & & & & & & \\
\hline O & & & & & & & & & & & & & & \\
\hline 0 & O & 0 & O & & & & & & & & & & & \\
\hline & & & & & & & & & & & & & & \\
\hline & & & & & & & & & & & & & & \\
\hline & & & & & & & & & & & & & & \\
\hline & & & & & & & & & & & & & & \\
\hline & & & & & & & & & & & & & & \\
\hline & & & & & & & & & & & & & & \\
\hline & & & & & & & & & & & & & & \\
\hline & & & & & & & & & & & & & & \\
\hline & & & & & & & & & & & & & & \\
\hline O & & & & & & & & & & & & & & \\
\hline & & & & & & & & & & & & & & \\
\hline & & & & & & & & & & & & & & \\
\hline 0 & 0 & O & 0 & 0 & 0 & 0 & 0 & 0 & & & & & & \\
\hline 705 & 705 & 704 & 675 & 614 & 524 & 469 & 407 & 319 & 234 & 189 & 180 & 173 & 156 & 120 \\
\hline 183 & 173 & 119 & 9 & 4 & 2 & 1 & 1 & 1 & 0 & 0 & 0 & 0 & 0 & 0 \\
\hline
\end{tabular}

Currently the maximum amount of Delta V available on a small satellite is below 500 $\mathrm{m} / \mathrm{s}$. This means that, at least for Cassini's case, impact of all of the moons is at least possible while orbiting any of the moons besides Titan with the current technologies does not seem likely. 


\section{ANCILLARY ANALYSIS}

As a bonus, the Mother-Daughter architecture allows for the exploration of objectives outside the scope of a specific mission. Cassini performed five major planetary flybys: Venus, Venus, Earth, Jupiter, and Saturn. While each flyby was essential to the mission as a whole, a plethora of other destinations were also achievable parallel to the primary directive by off shooting prior to a gravity assist maneuver.

Table 9. Missions to the Outer Planets [23]

\begin{tabular}{|c|c|c|c|c|c|}
\hline Spacecraft & Jupiter & Saturn & Uranus & Neptune & Pluto \\
\hline Pioneer 10 & 1973 flyby & & & & \\
\hline Pioneer 11 & 1974 flyby & 1979 flyby & & & \\
\hline Voyager 1 & 1979 flyby & 1980 flyby & & & \\
\hline Voyager 2 & 1979 flyby & 1981 flyby & $\begin{array}{l}1986 \\
\text { flyby }\end{array}$ & $\begin{array}{l}1989 \\
\text { flyby }\end{array}$ & \\
\hline Galileo & $\begin{array}{l}1995-2003 \text { orbit; } \\
1995,2003 \text { atmo. }\end{array}$ & & & & \\
\hline Ulysses & 1992,2004 flyby & & & & \\
\hline $\begin{array}{l}\text { Cassini- } \\
\text { Huygens }\end{array}$ & 2000 flyby & $\begin{array}{l}\text { 2004-now orbit; } \\
2005 \text { lander }\end{array}$ & & & \\
\hline $\begin{array}{c}\text { New } \\
\text { Horizons }\end{array}$ & 2007 flyby & & & & $\begin{array}{l}2015 \\
\text { flyby }\end{array}$ \\
\hline Juno & 2016-now orbiter & & & & \\
\hline
\end{tabular}

As mentioned in the first chapter, the amount of data gathered on other planets is a problem that needs to be solved before we can truly start to utilize the resources available in our solar system. As seen in Table 9 above, Cassini was only the seventh manmade satellite to interact with Jupiter and the fourth with Saturn. All of these encounters prior were brief flybys, except for Galileo, which orbited Jupiter from 1995-2003. Today there have only been three missions: Galileo, Cassini, and Juno that have orbited or impacted any of the outer planets.

This chapter will explore the possibility of using daughter spacecraft to collect data on the other planetary bodies that Cassini interfaced with along its journey. This analysis aims to reveal the possibilities available to such an architecture, whether the potential science is worth the cost can then be assessed depending on the desires and trade offs of the user. 


\subsection{Givens}

Table 10 below shows all of the information needed for the analysis of Cassini's interplanetary trajectory with regards to its start and end points as well as the four gravity assists.

Table 10. Planetary Data [22] [14]

\begin{tabular}{|c|c|c|c|c|c|}
\hline Planet & $\begin{array}{c}\text { Diameter } \\
(\mathrm{km})\end{array}$ & Mass $(\mathrm{kg})$ & $\begin{array}{c}\text { Semi-major } \\
\text { Axis }(\mathrm{km})\end{array}$ & Start Time (UTC) & End Time (UTC) \\
\hline Earth & 12,756 & $5.9722 \times 10^{24}$ & $149,600,000$ & $1997-$-Oct-15 08:43* & 1997-Oct-17 19:05 \\
\hline Venus & 12,104 & $4.8673 \times 10^{24}$ & $108,900,000$ & 1998-Apr-25 10:47 & 1998-Apr-27 16:43 \\
\hline Venus & 12,104 & $4.8673 \times 10^{24}$ & $108,900,000$ & 1999-Jun-24 02:43 & 1999-Jun-25 14:16 \\
\hline Earth & 12,756 & $5.9722 \times 10^{24}$ & $149,600,000$ & 1999-Aug-17 11:33 & 1999-Aug-18 19:23 \\
\hline Jupiter & 142,984 & $1.8982 \times 10^{27}$ & $778,600,000$ & 2000-Nov-9 22:26 & 2001-Feb-19 00:16 \\
\hline Saturn & 120,536 & $5.6832 \times 10^{26}$ & $1,433,500,000$ & 2004-Mar-12 09:22 & 2017-Sept-15* \\
\hline
\end{tabular}

Table 10 shows most of the data needed to perform analysis on the planets involved in the Cassini mission. The diameter identified for each planet is the equatorial (or maximum) diameter expressed in kilometers. For the gas giants with no definitive surface, this diameter was based off the altitude at which atmospheric pressure equals 1 atm. The mass and semi-major axis, expressed in kilograms and kilometers respectively, are not used in calculating Cassini's complex trajectory but are employed for the simple trajectories of potential CubeSat missions. The start and end time in Coordinated Universal Time (UTC) identifies when Cassini entered and exited the gravitational sphere of influence of each planet. The asterisks identify the launch of Cassini and its scheduled impact of Saturn.

Figure 22 below shows the timeline of Cassini's voyage including the dates that it entered and exited the planet's gravitational sphere of influence derived from Table 10. 


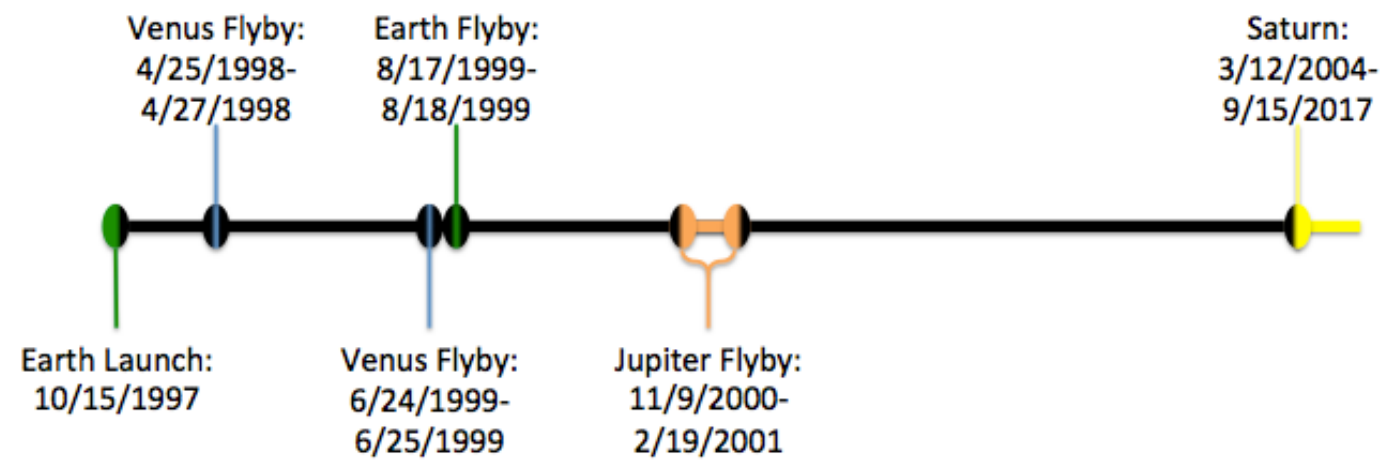

Figure 22. Cassini Timeline

Figure 23, derived using NASA's SPICE software, shows the actual trajectory as it was executed in three-dimensional space. This entire trajectory kept Cassini more or less flush with the plane of the solar system, until it reached Saturn. All of the interplanetary trajectories for this analysis, unless otherwise stated, are represented in the Heliocentric Ecliptic Coordinate System (J2000).

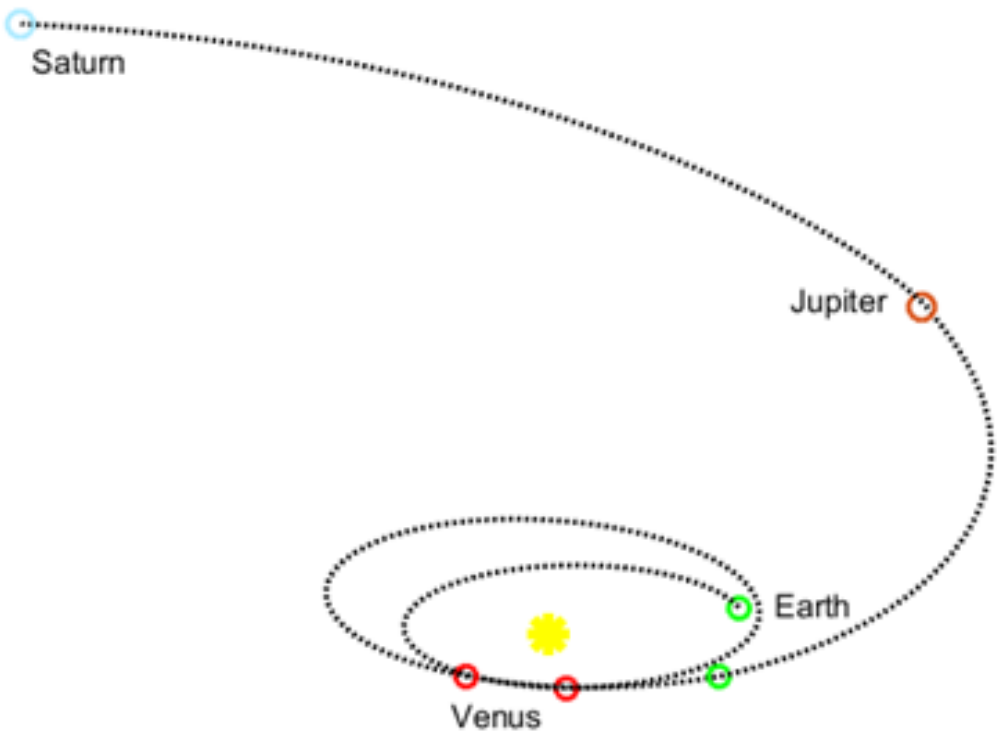

Figure 23. Cassini's Interplanetary Trajectory 


\subsection{Planetary Gravity Assists}

Each of Cassini's gravity assists were a necessity for a successful arrival to Saturn, but these gravity assists also could provide sufficient Delta V to allow for alternative pathways to other points of interest within the solar system and beyond.

Some of the major missions laid out in the 2013-2022 Decadal Survey, by leaders in the space industry include the following [24]:

- Uranus and Neptune/Triton

- Trojan and Kuiper belt object composition

- Comet/asteroid origin and evolution

- Studies of solar and heliospheric phenomena

- $\quad$ Primitive solar system bodies

By utilizing the architecture proposed in this section it may be possible to get daughter spacecraft to achieve the aforementioned missions as well as many other desirable missions that may arise in the future.

Many of these possibilities will be explored in this section using patched conics to address the possibility of individual planetary gravity assists for the daughter spacecraft. In order to facilitate this methodology, the problem was broken into three phases: the Deployment, the Gravity Assist, and the Drift.

\subsubsection{Process}

\subsubsection{Deployment Phase}

The Deployment phase takes the given trajectory of a mothership, in this case Cassini, from its exit of the previous gravitational sphere of influence to its entrance of the target planet's gravitational sphere of influence. Figure 24 below depicts the vector of possible deployment states in green. The orange shows the segments within the spheres of influence that were omitted. The gravitational sphere of influence for both the previous and target planet are depicted with black dashed lines. These were exaggerated significantly to better illustrate the range considered for deployment. 


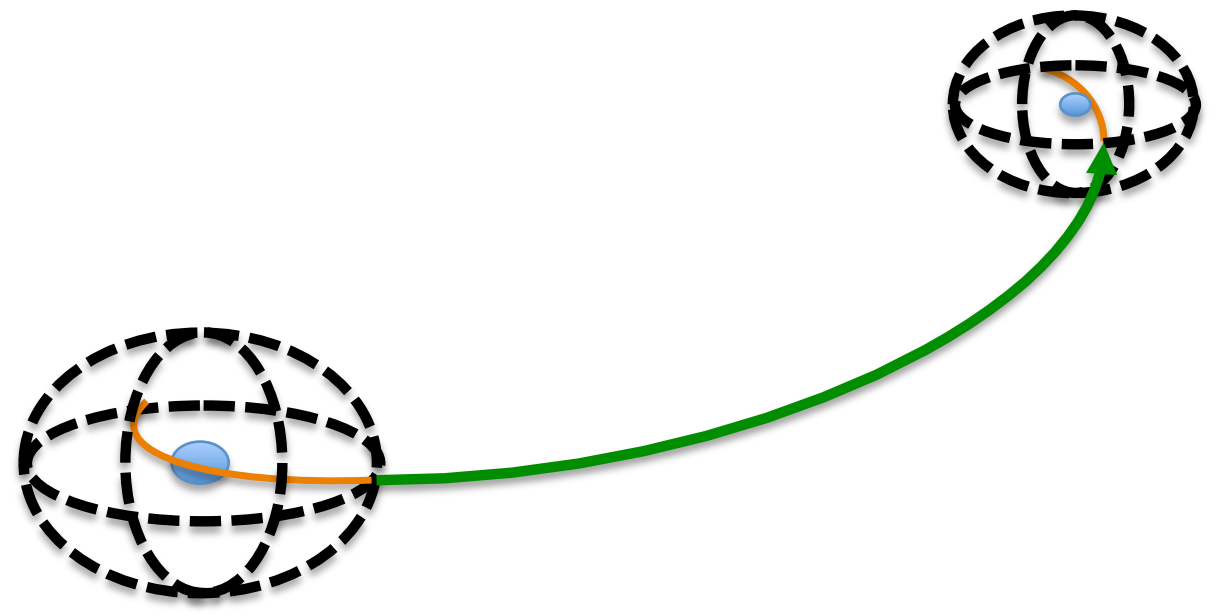

Figure 24. Daughter Spacecraft Deployment Range

This trajectory segment is then broken up into points of deployment evenly spaced with respect to time. The path of the target planet was also broken up into evenly spaced segments with respect to time. For this analysis, both segments were divided into 20 points of possibility. A 20 point analysis was decided upon to reduce computational strains while also provide enough points to discern patterns and trends as well as anchor basic models. A Lambert's solver was used to solve for the velocities needed to connect any two points of possibility in order to impact the target planet. The Lambert's solver used was the Izzo-Gooding Lambert's solver available in MatLab. The necessary deployment Delta V for each case was recorded. This deployment Delta V was the instantaneous difference between the velocity of the mothership at the given start point of possibility and the velocity needed by the Lambert's solution to impact the center of the target planet. The final step of the Deployment phase was to propagate forward each solution using a variable step Runge-Kutta 4th and 5th Order Method. MatLab's standard ode45 function was used for the variable step Runge-Kutta 4th and 5th Order Method. The propagation for each case was terminated at the edge of the target sphere of influence. In the event that the propagation did not terminate, the tolerances on the method were tightened and then reevaluated until termination. 


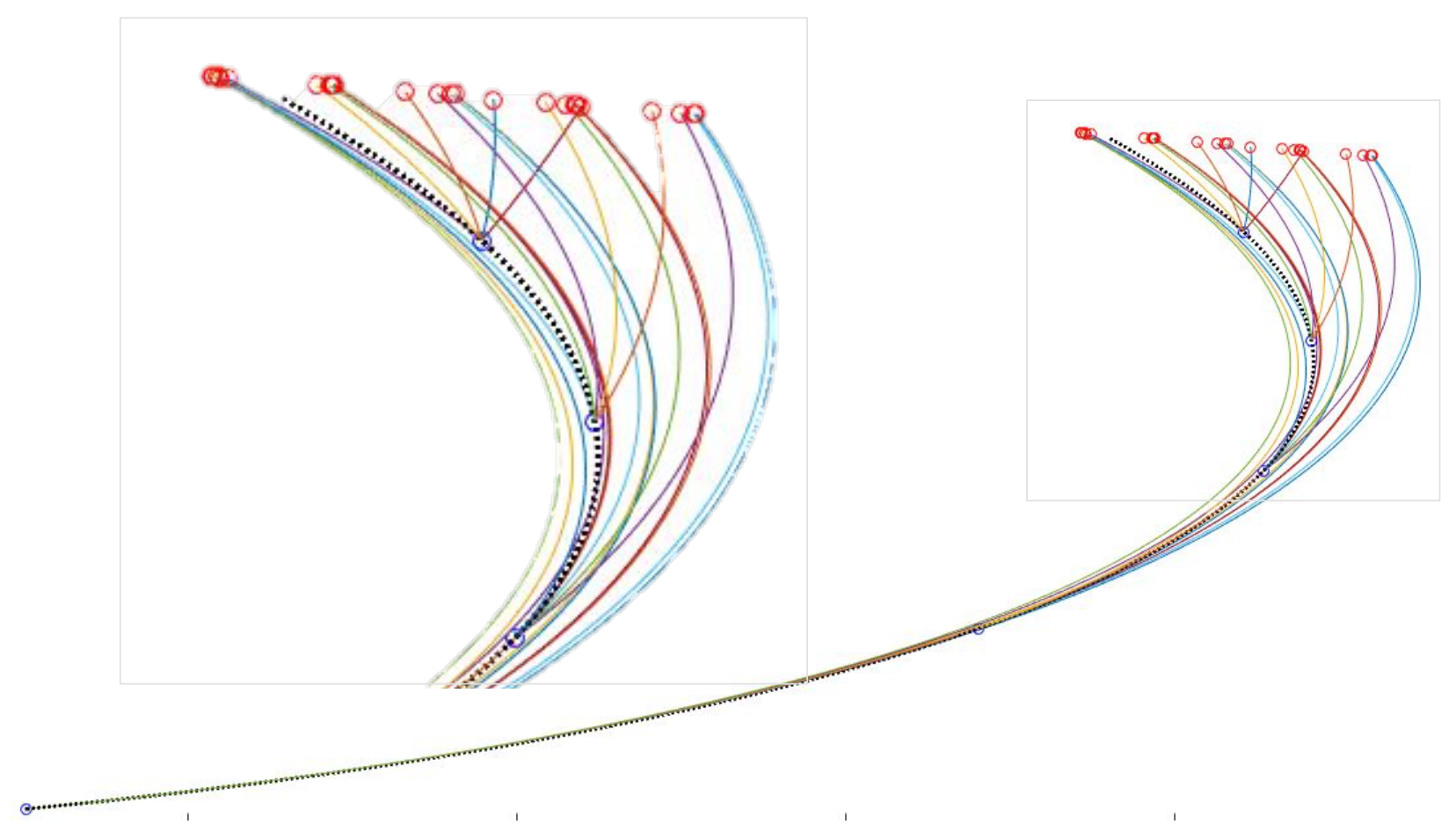

Figure 25. Simplified Jupiter SOI Entry

Figure 25 shows a less populated feather of possible deployments. The image in the box on the left is simply an enlargement of the image in the box on the right. Cassini's trajectory can be seen as the black dotted line. Each of the blue circles along Cassini's trajectory is a point of possible deployment that was considered. For this simplified illustration there were only 5 points of possible deployment where as in the actual analysis for each gravity assist there are 20. From each of these points of possible deployment a set of unique trajectories are propagated until they intersect the target's gravitational sphere of influence. Again, only 5 trajectories were propagated from each point of possible deployment for this example where as the actual analysis utilized 20. The termination of each daughter trajectory is indicated with a red circle.

\subsubsection{Gravity Assist Phase}

The Gravity Assist phase began by translating all of the possible states from the Heliocentric Ecliptic Coordinate System (J2000) to a planet centered reference frame depending on the target. This was primarily done by subtracting the states of the daughter spacecraft by the state of the target planet at that point in time. Figure 26 below shows a potential frame shift. The "spacecraft" and "planet" subscripts are represented in the interplanetary frame. $\mathrm{R}$ variables with 
the orange arrows are associated with the position vectors. The $\mathrm{R}_{\text {rel }}$ vector, originating at the center of the target planet, indicates the resulting relative position of the daughter spacecraft with respect to the planet. $\mathrm{V}$ variables with violet arrows indicate the velocity vectors of the target planet, daughter spacecraft, and the relative velocity of the daughter spacecraft. Again, the $V_{\text {rel }}$ vector was determined by subtracting the velocity of the daughter spacecraft by that of the planet. $\mathrm{V}_{\text {rel }}$ was expressed in two locations for simplicity.

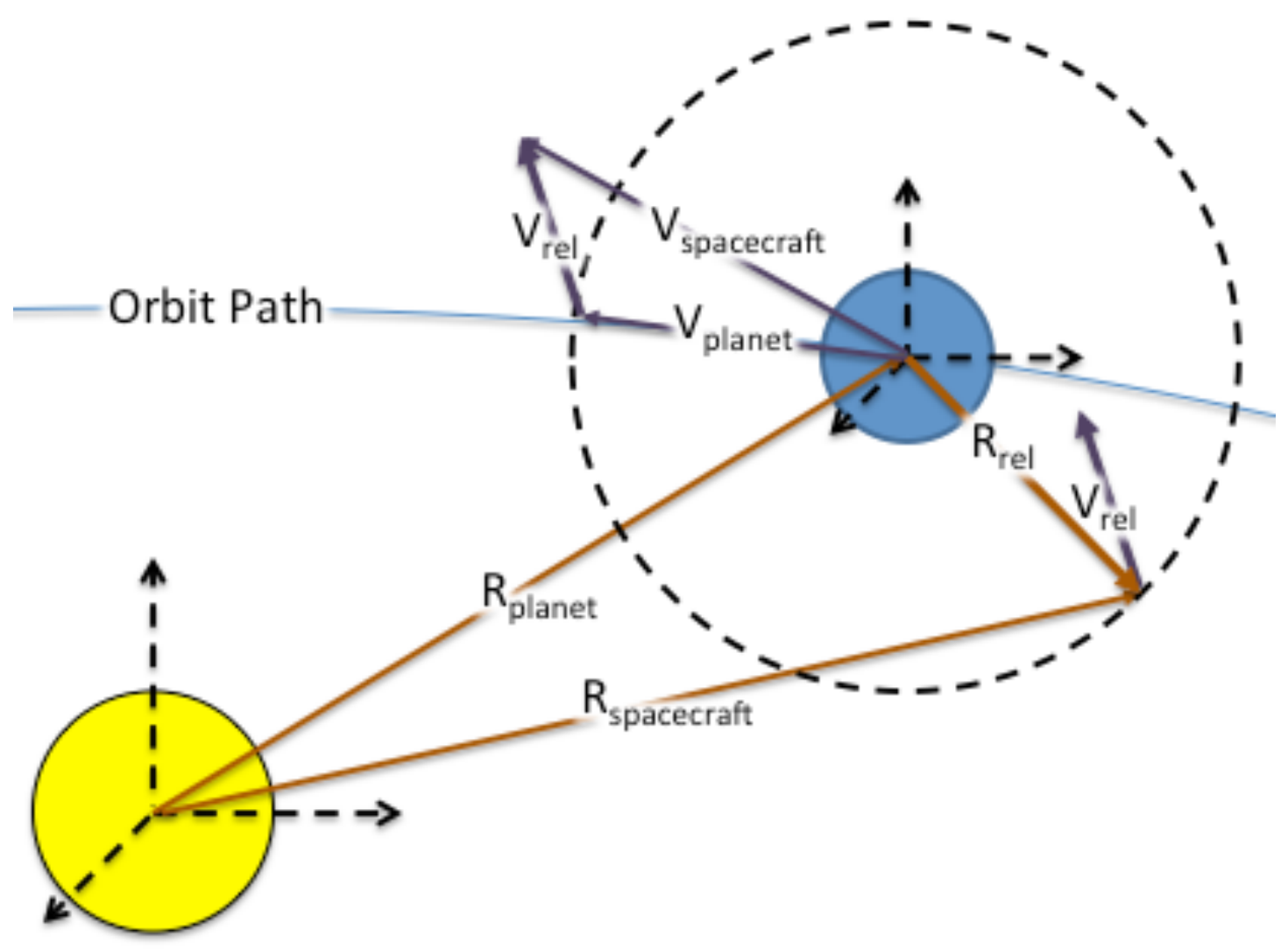

Figure 26. Interplanetary to Planetary Frame Shift

The next step was to create a locus of possible arrival trajectories. Figure 27 depicts a hypothetical locus of possibilities with labels for the relative velocity $\left(\mathrm{v}_{\infty}\right)$, Target circle, aiming radius $(\Delta)$, and Locus of periapses. 


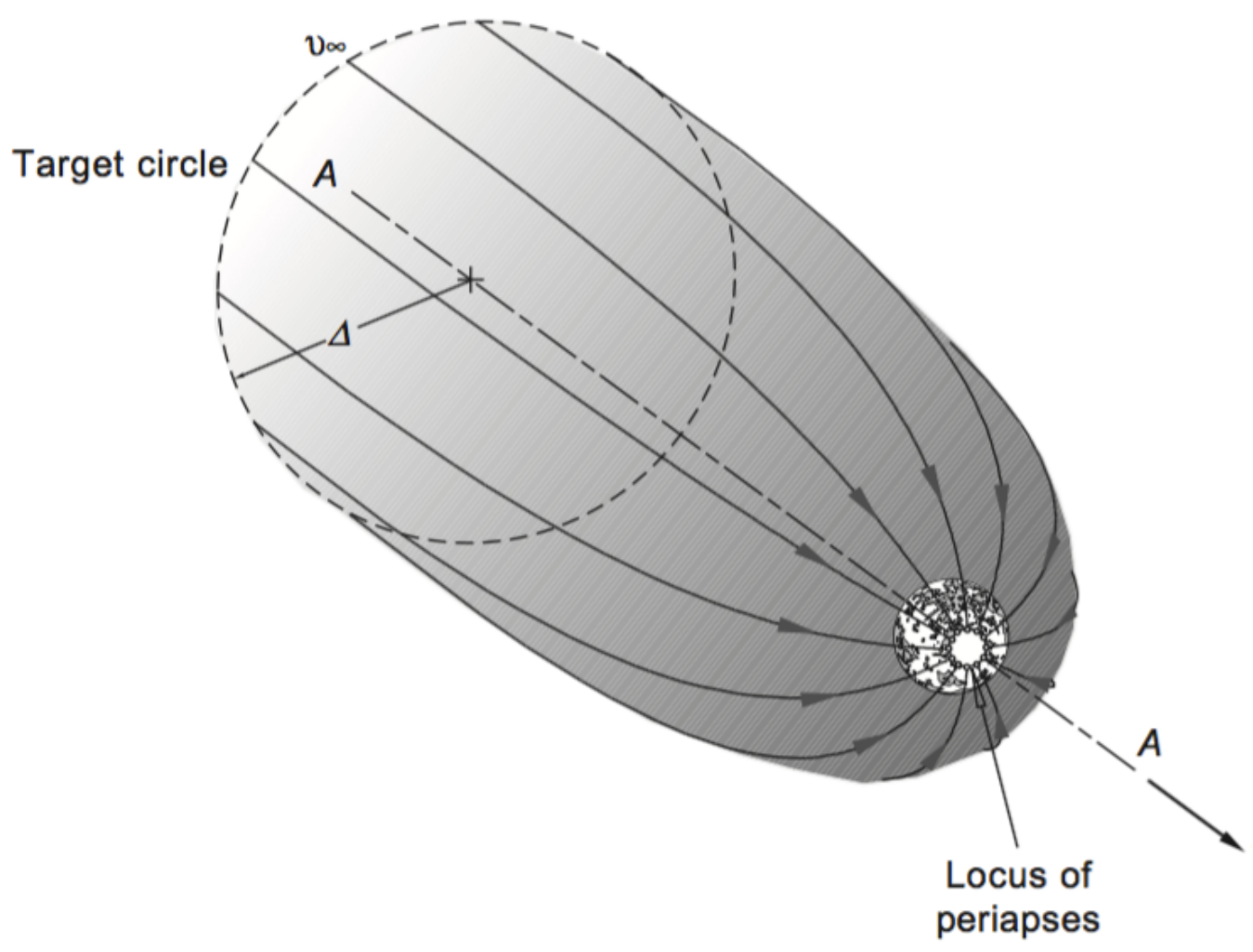

Figure 27. Locus of Possibilities [15]

This process begins by creating a target circle from the initial relative position and velocity. The radius of this target circle is created using the relative velocity of the spacecraft entering the gravitational sphere of influence $\left(\mathrm{v}_{\text {rel }}\right)$ along with a predetermined minimum altitude of periapse $\left(r_{p}\right)$ and the gravitational parameter $(\mu)$. These variables are used in Equation 4 . Aiming Radius to find the aiming radius $(\Delta)$, which represents the change in position needed in order to properly perform an effective gravity assist from the given initial state.

\section{Equation 4. Aiming Radius [15]}

$$
\Delta=r_{p} \sqrt{1+\frac{2 \mu}{r_{p} v_{\infty}^{2}}}
$$

The minimum altitude of periapse was used in order to maximize the Delta $\mathrm{V}$ achieved by any given gravity assist and was established at an altitude that would comfortably avoid the associated atmospheres of each of the planets. The actual numbers utilized to determine the aiming radius $(\Delta)$ can be seen in Table 11 below. The relative velocity at the gravitational sphere of 
influence $\left(\mathrm{v}_{\infty}\right)$ varied due to the deployment and arrival times determined in the Deployment phase.

Table 11. Planetary Gravity Assist Parameters [22]

\begin{tabular}{|c|c|c|c|c|}
\hline Planet & $\mu(\mathrm{km} 3 / \mathrm{s} 2)$ & $\begin{array}{c}\text { Minimum Altitude of } \\
\text { Periapse }(\mathrm{km})\end{array}$ & Radius $(\mathrm{km})$ & SOI $(\mathrm{km})$ \\
\hline Saturn & $3.79 \mathrm{E}+07$ & 2000 & 60268 & $5.46 \mathrm{E}+07$ \\
\hline Jupiter & $1.27 \mathrm{E}+08$ & 2000 & 71492 & $4.82 \mathrm{E}+07$ \\
\hline Earth & $3.99 \mathrm{E}+05$ & 300 & 6378 & $9.24 \mathrm{E}+05$ \\
\hline Venus & $3.25 \mathrm{E}+05$ & 300 & 6052 & $6.16 \mathrm{E}+05$ \\
\hline
\end{tabular}

$*^{\mu} \mu$ of the Sun is $1.32712 \mathrm{e} 11 \mathrm{~km} 3 / \mathrm{s} 2$

The radius of the gravitational sphere of influence (SOI) was found using the points of intersections between the magnitudes of gravity between the Sun and each planet as a function of distance from the Sun. The two distances of intersection became the diameter of the sphere of influence, in the same way done for the moons of Saturn. The gravitational sphere of influence can be seen in Figure 28 as the black sphere surface. The minimum altitude was decided on after researching the atmospheres of each planet and doing a basic regression to find a point where the density was less than $10^{-10} \mathrm{~km} / \mathrm{m}^{3}$ [25] [26].

A set of relative initial positions was created by shifting the initial position by the aiming radius in any direction perpendicular to the radius vector. By shifting the initial position evenly in all directions a target circle on the edge of the sphere of influence is created. This target circle is expressed to scale as a green circle in Figure 28. For this analysis, the circle was divided into 36 evenly spaced starting points, one point every $10^{\circ}$. This discretization gave an adequate distribution of points through the Drift Phase and again allowed for enough fidelity that patterns and models could be employed successfully. Each of these points was used to create a unique state by coupling it with the initial relative velocity of the spacecraft entering the gravitational sphere of influence $\left(\mathrm{v}_{\infty}\right)$. Although this forced coupling will not account for the entire Delta $\mathrm{V}$ needed for the maneuver, the amount unconsidered was negligible and the solution for this nuance will not serve to better validate these possibilities. 


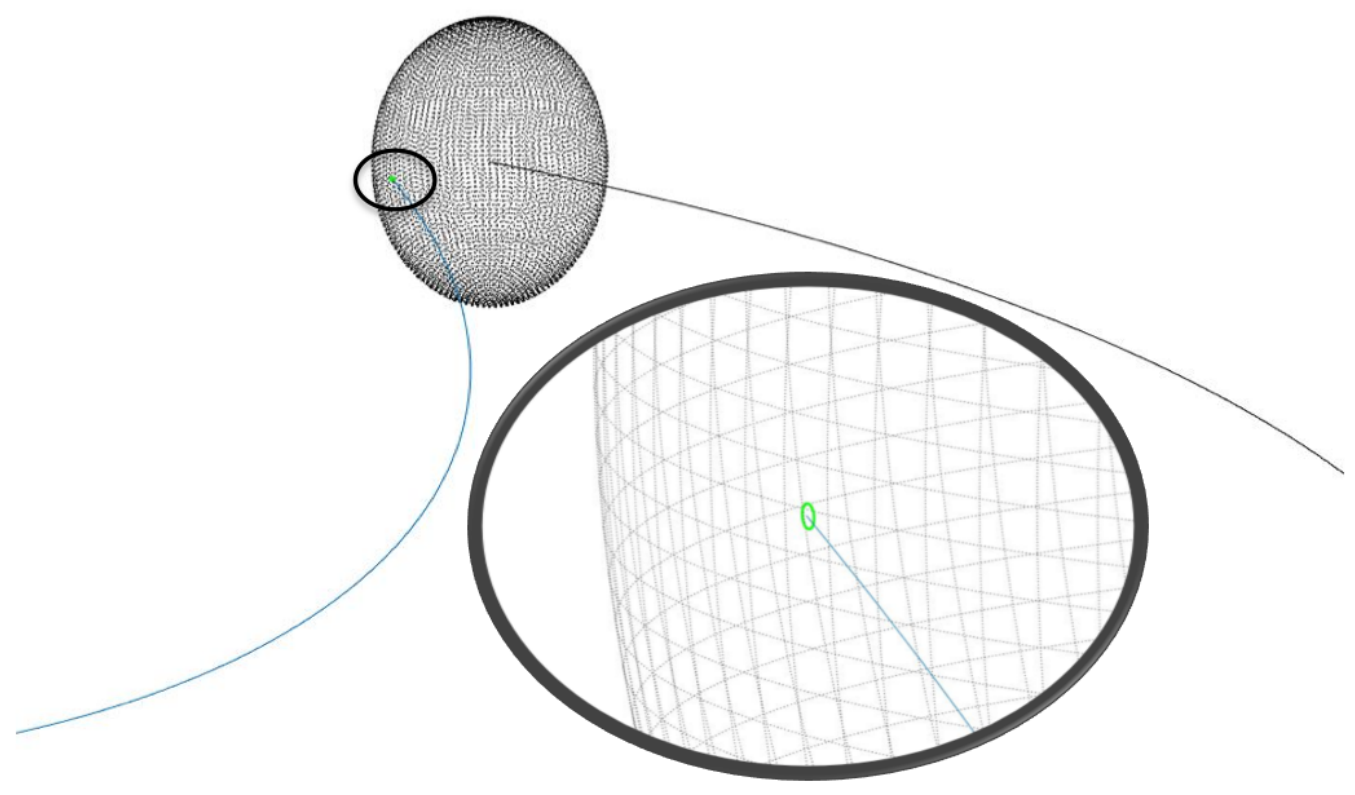

Figure 28. Target Circle and Gravitational Sphere of Influence

Compared to the Delta V and distances of the initial deployment phase this aiming radius was negligible for all cases. The actual precision alignment of the entry states is possible using advanced optimization and iteration processes; however, as demonstrated in Figure 29 below, for the purpose of this study the use of patched conics was sufficient.

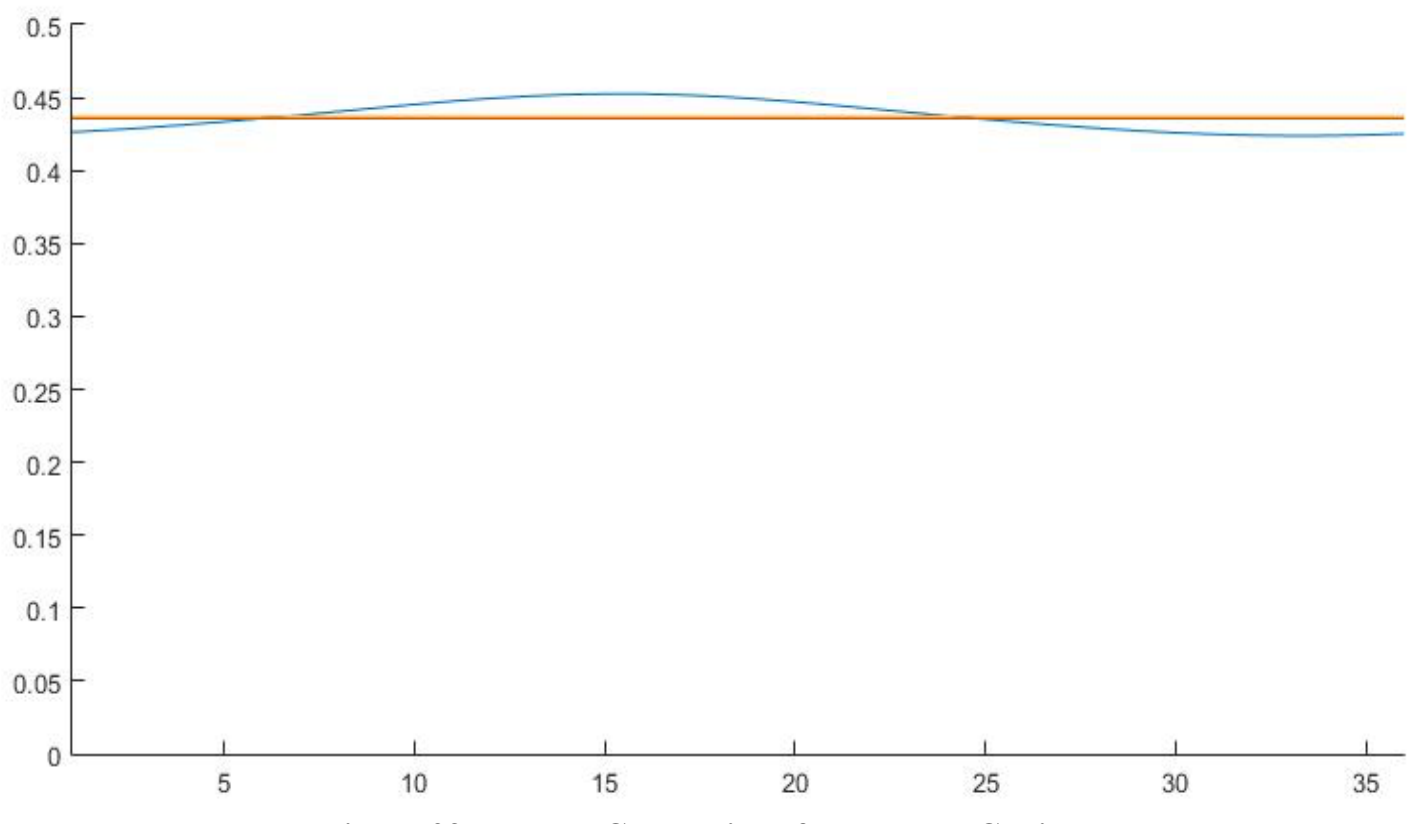

Figure 29. Delta V Comparison for Patched Conics 
To further justify the use of patched conics, there are three plots in Figure 29 each of which demonstrates a different Delta V accuracy. This study was performed on one of Jupiter's entry trajectories, but can be replicated for all other gravity assists. On the vertical axis is the required Delta $\mathrm{V}$ in kilometers per second and on the horizontal is the number of the locus point $(1-36)$

The blue curve represents the Delta $\mathrm{V}$ required to reach each individual point on the target circle and have the relative velocity equal to that of the daughter spacecraft entering the center of the circle. This was achieved by using another Izzo-Gooding Lambert's solver to connect the point of deployment to each point on the target circle individually. Because of this, some sections of the blue curve require less Delta V since they are closer to Cassini's point of entry than others. The final velocity from the Lambert's solver was subtracted from the relative velocity of the original daughter spacecraft at the center of the circle to ensure that a second burn could force all of the states around the target circle to have the same relative velocity. This method forces a solution to the discontinuity of patched conics.

The other two plots are horizontal since they are both averages. The yellow line is the average of the blue curve and the red line barely below it is the patched conics assumption. The reason that the patched conics method slightly underestimates the Delta $\mathrm{V}$ was because of the second burn used to force all of the plots to have a homogenous relative velocity, with out the second burn the average of the blue line would be equal to that of the patched conic method which proves that for the purpose of aiding early daughter spacecraft design patched conics was an adequate method.

The final step in the Gravity Assist phase can be seen in Figure 30 below and is again to propagate forward each solution in time using a variable step Runge-Kutta 4th and 5th Order Method. The propagation for each case is terminated (red circles) at the exit of the target sphere of influence. 


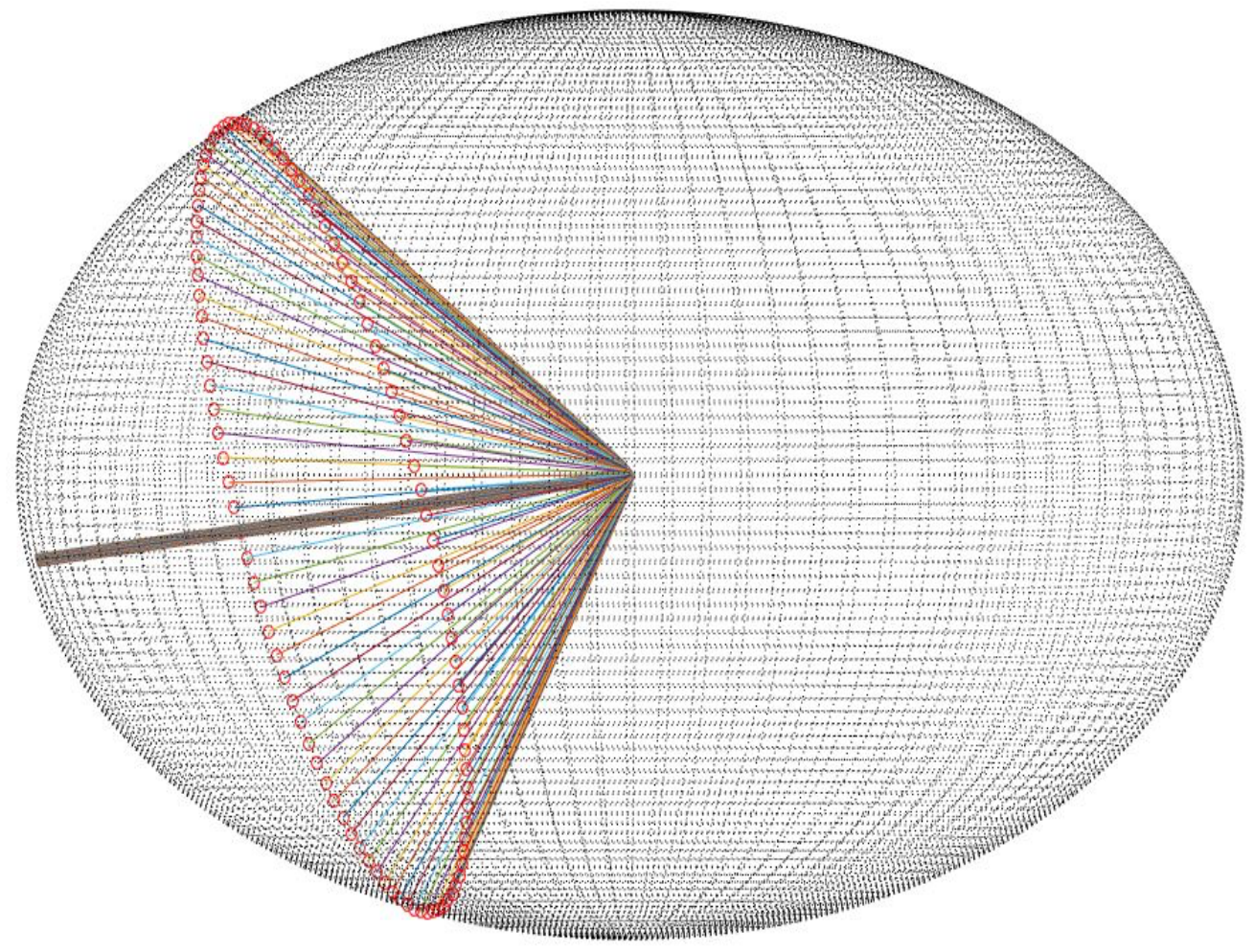

Figure 30. Gravity Assist Locus in the Planet Centered Frame

Figure 30 above shows a single gravity assist around Jupiter with a set of different possible exit states. In this example, 100 possibilities were used where as in the actual analysis 36 points were deemed sufficient. It can be seen that each of these trajectories starts from a similar location, the target circle and then after passing through the radius of periapse achieves a different exit position and direction, seen as the red circle.

\subsubsection{Drift Phase}

The Drift phase begins by converting frames from the relative planet centered frame back to the Heliocentric Ecliptic Coordinate System (J2000) for the various exit states from the end of the Gravity Assist phase. This was done by adding the state of the planet at the time of exit to the state of the daughter spacecraft. For a few points in time there is a lack of planetary state data. To address this issue a spline interpolation based on the most recent planetary state data was used as an approximation. This initial state was then used to propagate forward each solution using a variable step Runge-Kutta 4th and 5th Order Method. Each case is propagated for a determined 
amount of time, for this analysis it was 10 years after deployment from the mothership. This timespan well overshoots the current capabilities of small satellites, but allows for a thorough investigation at minimal cost to computational strain.

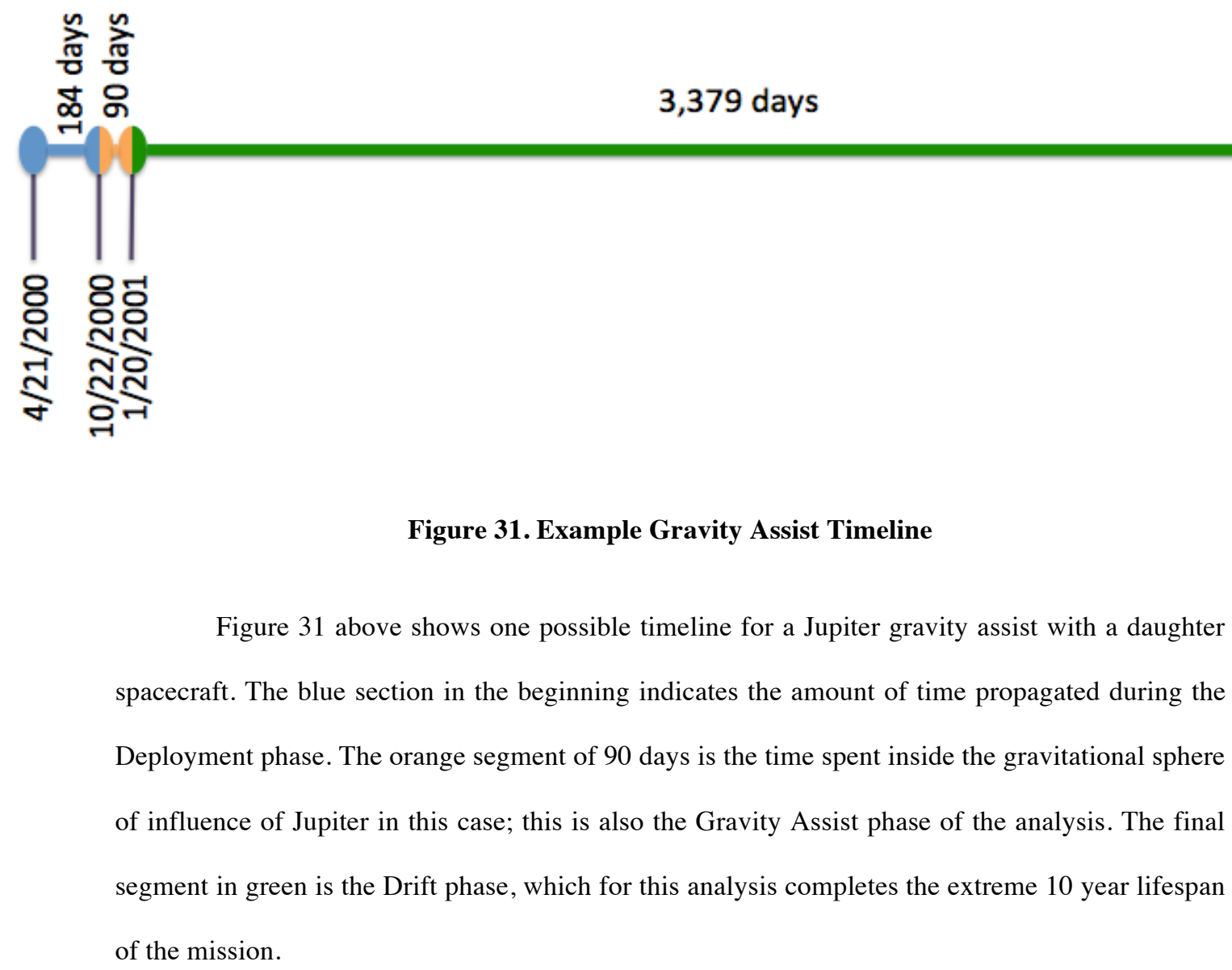




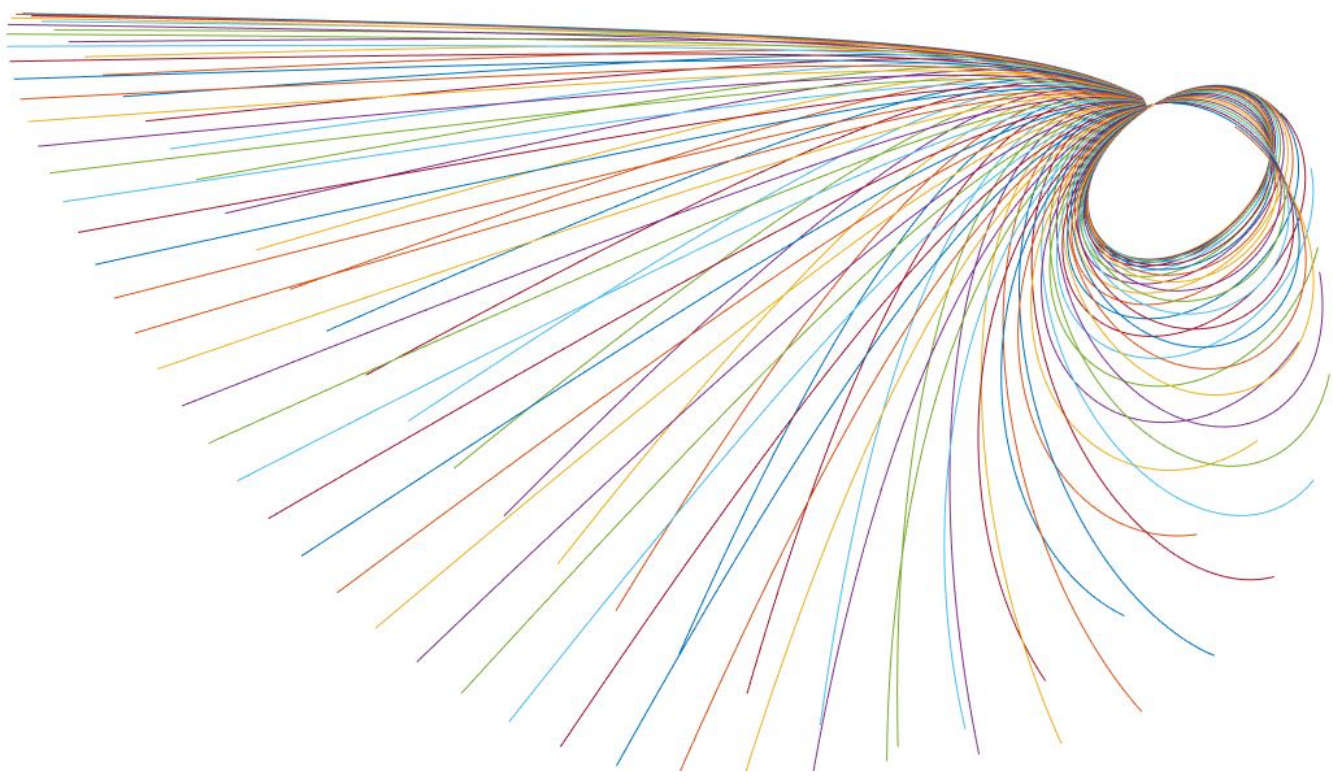

Figure 32. Gravity Assist Propagation (15 years)

Figure 32 above shows the propagation of the Jupiter gravity assist seen in Figure 30 earlier. The time span for this propagation was 15 years (rather than 10) to better show the various possible trajectories, where most small satellites will only be designed to survive the first year. The two major categories seen are the escape trajectories located further to the left and the solar orbiters seen on the far right of Figure 32 above.

\subsubsection{Results}

The following results were achieved using 20 initial deployment positions evenly spaced in time, 20 arrival positions also evenly distributed with respect to time, and a 36 point target circle for generating loci of possibilities for all five flybys over a 10-year lifespan. These discretization provided a substantial matrix of cases by which to determine and optimize an initial trajectory for a planetary flyby with a swath of customizable parameters.

Figure 33 and Figure 34 below show the two distinct categories of gravity assist trajectories for a 10-year mission lifetime. Figure 33 shows the results for all of the trajectories after exiting Venus's gravitational sphere of influence in 1998. It can be seen that regardless of exit orientation the trajectories are always elliptical. This is true for the first three gravity assists 
for Cassini, Venus 1998, Venus 1999, and Earth 1999, since none of these planets can create enough Delta V alone to reach escape velocity from the solar system.

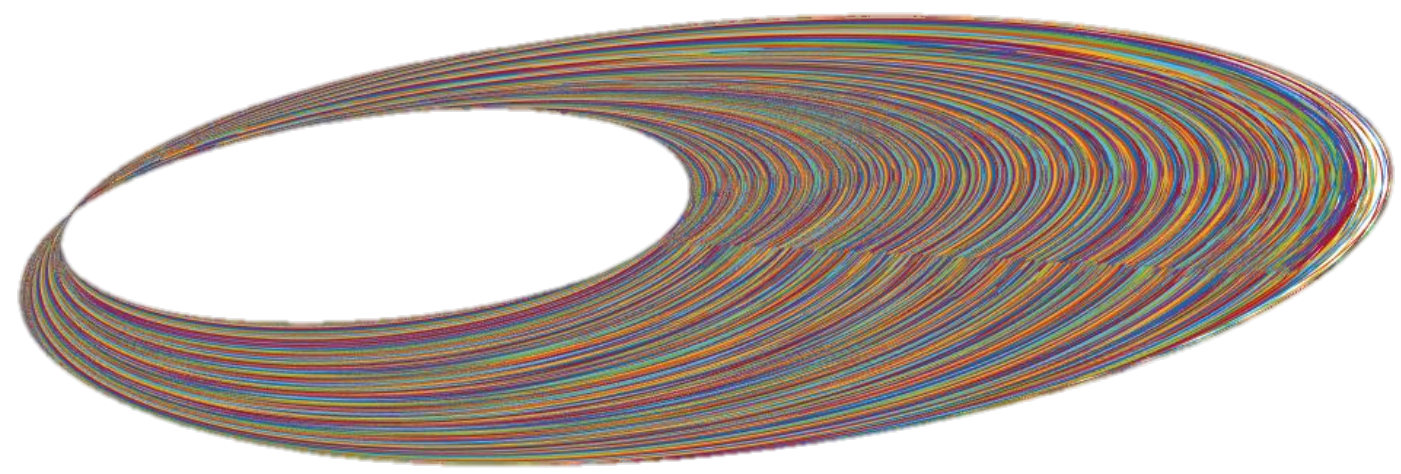

Figure 33. Venus 1998 Gravity Assist Possibilities

Figure 34 illustrates the other possible result from the gravity assist analysis where some of the trajectories actually receive enough of a Delta $\mathrm{V}$ boost to exit the solar system. This occurred during both the Jupiter 2000 and Saturn 2004 gravity assists. Some of the other trajectories remained trapped by the gravity of the Sun and are thus large elliptical orbits as seen on the right side.

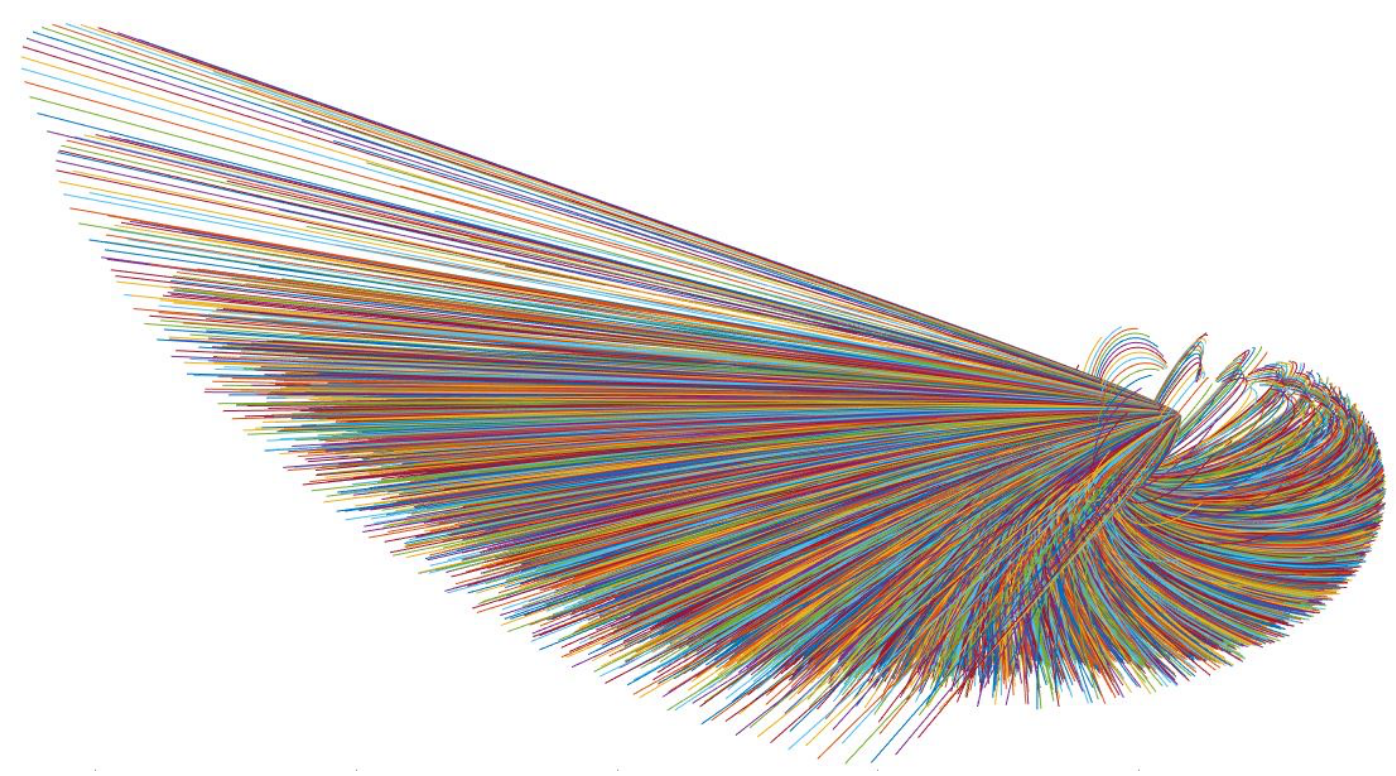

Figure 34. Jupiter 2000 Gravity Assist Possibilities 
By evaluating the parameters for all of the gravity assists a set of plots can be created to identify the trends prevelant in the data. Figure 35 and Figure 36 below show the inclination and distance achievable with respect to the available Delta V.

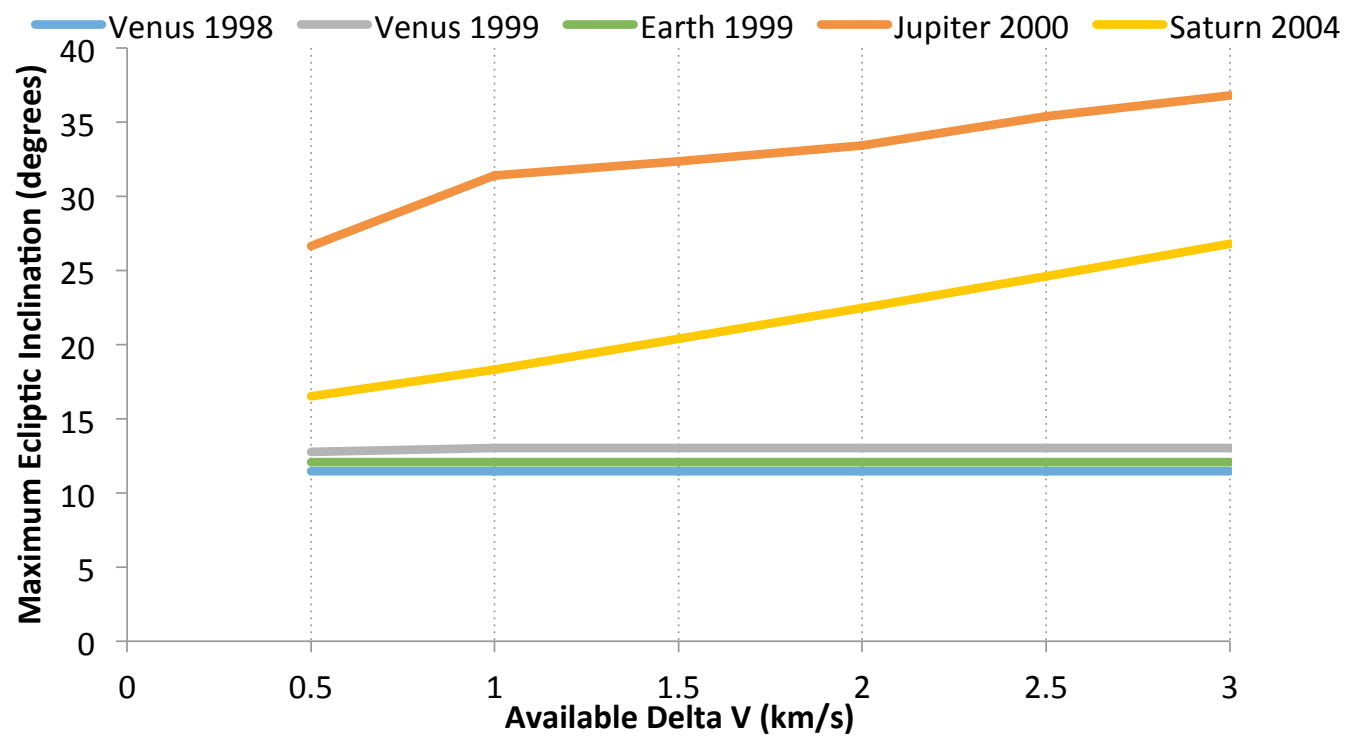

Figure 35. Maximum Inclination from the Ecliptic

Figure 35 shows the maximum inclination that a daughter spacecraft can achieve by utilizing one of the given gravity assists to attain a different trajectory. The Delta $\mathrm{V}$ in kilometers per second is the amount of fuel required to instantaneously separate from Cassini's trajectory and enter into the target planet's gravitational sphere of influence at a different point. The results in terms of degrees inclination were measured as an angle off of the Earth-Sun Ecliptic plane. For perspective the highest inclination that a spacecraft has attained was the joint ESA/NASA Ulysses mission, which reached $80.2^{\circ}$ inclination [27] 


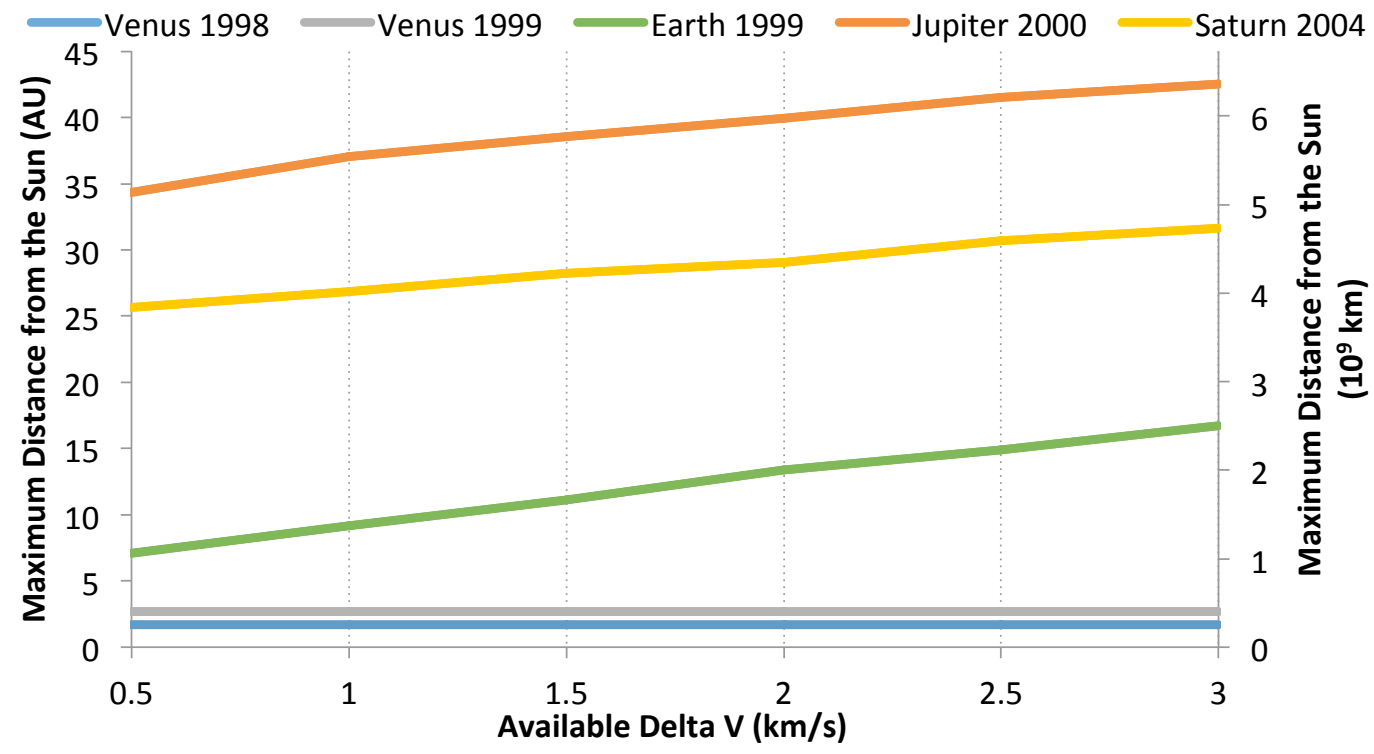

Figure 36. Maximum Distance from the Sun After 10 Years

Figure 36 shows the potential distance from the Sun after 10 years of deployment with respect to the amount of Delta V in kilometers per second onboard the daughter spacecraft. The left vertical shows the maximum distance from the Sun in astronomical units (149597870.7 kilometers) and the right vertical shows the maximum distance in billions of kilometers. For perspective the furthest spacecraft from Earth, Voyager 1, is currently 137 AU from the Sun [28].

By utilizing the Jupiter 2000 gravity assist the minimum Delta V required to escape was $354 \mathrm{~m} / \mathrm{s}$ when deploying on 11/16/1999 15:12:54 UTC. This would involve 355 days of transit prior to entering Jupiter's gravitational sphere of influence and then another 98 days before exiting out into interplanetary space. After 10 years this spacecraft would be approximately 4,920,518,057 kilometers (32.9 AU) from the Sun. By using the Saturn 2004 gravity assist the minimum Delta V required to escape was $24 \mathrm{~m} / \mathrm{s}$ when deploying on 6/10/01 18:00 UTC. This would involve 1,000 days of transit prior to entering Saturn's gravitational sphere of influence and then another 224 days before exiting out into interplanetary space. After 10 years this spacecraft would be approximately 2,071,000,000 kilometers (13.8 AU) from the Sun. 


\subsection{Summary}

While there does exist optimized methods for calculating maximum inclination or velocity given a specified flyby, the ancillary analysis performed above provides a wide range of possible trajectories that can be used to find a specific mission and attain rough numbers given a quick table lookup. Table 12 shows a few of the cases optimized for a single parameter like minimum Delta V. This process provides a good "ball park" approximation for the capabilities on a wide array of mission cases, from which a more thorough investigation can be conducted.

Table 12. Augmented Cassini's Planetary Possibilities

\begin{tabular}{|c|c|c|c|}
\hline Planet & $\begin{array}{c}\text { Min } \Delta \text { V Impact } \\
(\mathrm{m} / \mathrm{s})\end{array}$ & $\begin{array}{c}\text { Max } \mathrm{R}_{\text {sun }} \\
@ 1 \mathrm{~km} / \mathrm{s} \\
\left(10^{9} \mathrm{~km}\right)\end{array}$ & $\begin{array}{c}\text { Max Inc } \\
@ 1 \mathrm{~km} / \mathrm{s} \\
(\mathrm{deg})\end{array}$ \\
\hline Venus & 1 & 0.4 & 13.0 \\
\hline Earth & 13 & 1.4 & 12.1 \\
\hline Jupiter & 354 & 5.5 & 31.4 \\
\hline Saturn & 24 & 4.0 & 18.2 \\
\hline
\end{tabular}




\section{CONCLUSION}

There is currently a lack of information on most of the solar system, resulting in complex speculations and mathematical models to answer questions beyond our neighboring planets. The simple solution would be to send more missions out to sites beyond Mars and Venus, but this would require resources currently unavailable to the industry. As such there is a need for more in situ data on far off locations that stem from a single efficient mission. The Mother-Daughter mission architecture is likely to be the first step to solving this problem. By attaching smaller daughter spacecraft to a mothership bound for a distant location, one can effectively doubled the number of attainable data locations. The more augmented the mothership is with daughter spacecraft the more far reaching that mission will become. While previous missions focused on the quality of a select amount of data points, a new trend is beginning to take shape, which focuses on larger quantities of data to establish better models.

Cassini was chosen as a well-understood case from which to base the Mother-Daughter architecture analysis. This allowed a more practical application of this new architecture rather than purely speculation, and while this thesis focused on Cassini and its trajectory, the processes used can easily be applied to other missions in the future with similar results.

The trajectories attainable using a Mother-Daughter architecture heavily vary with the amount of Delta $V$ that the daughter spacecraft possess, so a large number of desirable cases were generated in this analysis.

Once inside the Saturnian System a plethora of opportunities become available to a Mother-Daughter Architecture. One of these options, Saturn's rings can be targeted by simply separating from the mothership in a way that lowers the altitude of periapse efficiently. Through some basic optimization of Cassini's trajectory with respect to Saturn, it was found that impacting Saturn's rings could be done with 200 - 800 meters per second of separation Delta V depending on which ring was desired. Other methods of impact were explored, but resulted in less desirable trajectories. The primary analysis on the moons in the Saturnian System. The analysis looked at the 23 moons explored during the Cassini mission and explored possibilities during all of the flybys to date. Out of Cassini's 709 moon flyby cases considered 614 (87\%) would allow for an 
impactor with $1 \mathrm{~km} / \mathrm{s}$ Delta V. At $.1 \mathrm{~km} / \mathrm{s}$ there are 234 (33\%) potential impact cases. Daphnis, the most difficult moon to impact in Cassini's trajectory, would require a minimum of $206 \mathrm{~m} / \mathrm{s}$ of Delta V while the impact of Titan would only require $1 \mathrm{~m} / \mathrm{s}$ if performed at the correct time. The study of this specific case indicated that by simply increasing the separation Delta $\mathrm{V}$ to $3 \mathrm{~m} / \mathrm{s}$ the window of opportunity is around 8 hours. Only 5 of Saturn's moons have their own gravitational spheres of influence. Hyperion, Iapetus, Phoebe, Rhea, and Titan are the only moons in the Saturnian System in which orbit can be attained without the use of relative motion around Saturn. Of these five moons, Phoebe was the most difficult moon to orbit in Cassini's trajectory with a minimum of $6.25 \mathrm{~km} / \mathrm{s}$ of Delta $\mathrm{V}$ needed to orbit while Titan, the most massive and visited of the moons, would only require $0.12 \mathrm{~km} / \mathrm{s}$ if the maneuvers were performed at the correct time. Flybys can also be achieved by varying the direction, Delta V, or release time of separation.

Ancillary analysis, outside the scope of the Cassini directive looked at the possibility of utilizing gravity assists that deviated from that of the mothership in order to escape the solar system or incur a plane change. After analyzing 14,400 unique cases per each of the five planetary gravity assists, the maximum distance from the sun after a decade and the inclination from the ecliptic attainable clearly occurred by utilizing Jupiter's gravity field. With 1 kilometer per second of Delta V a daughter spacecraft could be 5,539,400,000 km (37.029 AU) from the Sun after a tenyear lifespan or could change its inclination to $31.4^{\circ}$.

The exploration of these gravity assist planets is also a possibility. By using a daughter spacecraft it would be theoretically possible to flyby or impact any of these planets. The analysis shows that orbit through the means of aerobraking would be extremely difficult due to the level of precision required and the fundamental simplicity of a daughter probe. This aerobraking analysis did however show the elevations that differentiate a low altitude flyby from an atmospheric capture or impact for a specific geometry. This analysis could easily be tweaked for more specific cases but demonstrates that both impacting and performing low altitude flybys of every planet is in fact feasible.

The high level results can be found in Table 13 below. 
Table 13. Augmented Cassini Possibilities

\begin{tabular}{|c|c|c|c|c|c|c|c|c|}
\hline & \multicolumn{4}{|c|}{ Interplanetary Space } & \multicolumn{4}{c|}{ Saturnian System } \\
\hline S/C $\Delta \mathrm{V}$ & Flybys & Impacts & $\begin{array}{c}\text { Max } \mathrm{R}_{\text {sun }} \\
\left(10^{9} \mathrm{~km}\right)\end{array}$ & $\begin{array}{c}\text { Max Inc } \\
(\mathrm{deg})\end{array}$ & Flybys & Orbits & Impacts & $\begin{array}{c}\text { Lowest } \\
\text { Ring }\end{array}$ \\
\hline $1 \mathrm{~m} / \mathrm{s}$ & All & Venus & N/A & N/A & All & None & $1(4 \%)$ & None \\
\hline $10 \mathrm{~m} / \mathrm{s}$ & All & Venus & N/A & N/A & All & None & $7(27 \%)$ & None \\
\hline $100 \mathrm{~m} / \mathrm{s}$ & All & $\begin{array}{c}\text { Venus } \\
\text { Saturn }\end{array}$ & N/A & N/A & All & None & $18(69 \%)$ & None \\
\hline $500 \mathrm{~m} / \mathrm{s}$ & All & All & 5.1 & 27 & All & Titan & All & B Ring \\
\hline $1 \mathrm{~km} / \mathrm{s}$ & All & All & 5.5 & 31 & All & Titan & All & All \\
\hline $2 \mathrm{~km} / \mathrm{s}$ & All & All & 6.0 & 33 & All & $\begin{array}{c}\text { Titan } \\
\text { Iapetus }\end{array}$ & All & All \\
\hline $3 \mathrm{~km} / \mathrm{s}$ & All & All & 6.4 & 37 & All & $\begin{array}{c}\text { Titan } \\
\text { Iapetus }\end{array}$ & All & All \\
\hline
\end{tabular}

The analysis used in this thesis and future analysis like it, serve as a strong platform from which to explore the variety of that the Mother-Daughter architecture has to offer the future of space exploration. The requirements found through these various cases can be utilized as an initial baseline for the design of a specific mission of interest, while more directed analysis could help in polishing the baseline in series with the design process. The next steps toward improving our understanding of the solar system would be a similar study of a future interplanetary mission, the design of specific missions for the daughter spacecraft, and collaboration and consent from the designers of the mothership. 


\section{BIBLIOGRAPHY}

[1] Sean McNaughton and Matt Twombly. (2014, May) National Geographic. [Online]. http://www.5wgraphics.com/img/newsletter/50-years-of-exploration.jpg

[2] NASA JPL, A-Team Meeting: The Growth of CubeSats, July 21, 2016.

[3] Henry Helvajian and Siegfried W., eds. Janson, Small Satellites: Past, Present, and Future. El Segundo: Aerospace Press, 2008.

[4] Radius Space. [Online]. www.radiusspace.com

[5] M. A. Swartwout. (2016, July) CubeSat Database. [Online]. https://sites.google.com/a/slu.edu/swartwout/home/cubesat-database

[6] National Academies of Sciences, Engineering, and Medicine, Achieving Science with CubeSats: Thinking Inside the Box. Washington, DC, United States of America: The National Academies Press, 2016.

[7] Klint Finley. (2012, August) NASA Lets You Build Your Own Satellite with PhoneSat. [Online]. http://www.wired.co.uk/article/nasa-satellite-android

[8] Phillips Davis. (2016, September) National Aeronautics and Space Administration. [Online]. https://saturn.jpl.nasa.gov/mission/

[9] National Aeronautics and Space Administration. (2000) Solar System Exploration: Planets. [Online]. http://nssdc.gsfc.nasa.gov/planetary/factsheet/

[10] The Navigation and Ancillary Information Facility. (2016, July) Cassini Orbiter Archive. [Online]. http://naif.jpl.nasa.gov/pub/naif/pds/data/co-s_j_e_v-spice-6-v1.0/cosp_1000/

[11] Howard Curtis, Orbital Mechanics for Engineering Students, Second Edition ed. Burlington, USA: Elsevier, 2010.

[12] C. G. Justus and R. D. Braun, "Atmospheric Environments for Entry, Descent and Landing (EDL)," Natural Environments Branch (EV13), NASA Marshall Space Flight Center, 2007.

[13] Alvin Seiff et al., "Structure of the Atmosphere of Jupiter: Galileo Probe Measurements," Science, New Series, vol. 272, no. 5263, pp. 844-845, May 1996. [Online]. 
http://www.jstor.org/stable/2890205

[14] Kenneth Moe and Mildred M. Moe, "Gas-Surface Interactions and Satellite Drag Coefficients," Planetary and Space Science, vol. 53, no. 2005, pp. 794-801, March 2005.

[15] Justin Foley, "6U CubeSat Design Specifiation Rev. Provisional," CubeSat, San Luis Obispo, Design Specification 2016.

[16] Bob Pappalardo, Cassini Proposed Extended-Extended Mission (XXM), March 9, 2009.

[17] R. A. Jacobson et al., "The Gravity Field of the Saturnian System from Satellite Observations and Spacecraft Tracking Data," The Astronomical Journal, vol. 132, no. 6, pp. 2520-2526, 2006.

[18] P. C. Thomas, "Sizes, Shapes, and Derived Properties of the Saturnian Satellites After the Cassini nominal Mission," Icarus, vol. 208, no. 1, pp. 395-401, 2010.

[19] NASA JPL, Saturn's Rings Dark Side Mosaic, October 20, 2007, Image from the Cassini Mission.

[20] Daniel Andrews. (2010, October) National Aeronautics and Space Administration. [Online]. http://lcross.arc.nasa.gov/

[21] Armen Toorian, "CubeSats: From Launch to Deployment," in CubeSat Developers' Workshop, San Luis Obispo, 2003.

[22] Busek: Space Propulsion and Systems. (2016, September) Busek. [Online]. http://www.busek.com/cubesatprop__main.htm

[23] Planet Labs Inc. (2016, September) Planet. [Online]. https://www.planet.com/

[24] PolySat. (2014) PolySat. [Online]. http://polysat.calpoly.edu/launched-missions/cp10exocube/

[25] David R Williams. (2016, May) Saturn Fact Sheet. [Online]. http://nssdc.gsfc.nasa.gov/planetary/factsheet/saturnfact.html

[26] J. L. Simon et al., "Numerical expressions for precession formulae and mean elements for the Moon and the planets," in Astronomy and Astrophysics. Paris: Bureau des Longitudes, 1994, 
pp. 663-683.

[27] Tibor Balint, "Summary of Russian Planetary Lander Missions," Deep Space Mission Architecture, 2002.

[28] Arash Mehrparvar, "CubeSat Design Specification Rev. 13," San Luis Obispo, 2014.

[29] Mars Exploration: Program \& $\quad \& \quad$ Missions. $\quad$ [Online]. http://mars.jpl.nasa.gov/programmissions/missions/past/mariner89/ 


\section{APPENDIX}

Impactor Data

\begin{tabular}{|c|c|c|c|c|c|c|c|c|c|c|c|c|c|c|c|c|c|c|c|}
\hline Moon & 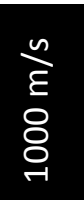 & 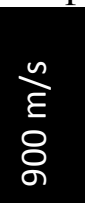 & 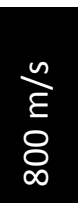 & $\begin{array}{l}\stackrel{n}{\xi} \\
\stackrel{\circ}{尺}\end{array}$ & $\begin{array}{l}\frac{n}{E} \\
8 \\
\varnothing\end{array}$ & 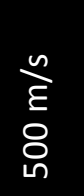 & $\begin{array}{l}\stackrel{n}{\xi} \\
\stackrel{\sigma}{\sigma}\end{array}$ & 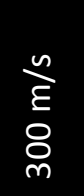 & $\begin{array}{l}\stackrel{n}{\xi} \\
\stackrel{్}{\delta}\end{array}$ & $\begin{array}{l}\stackrel{n}{E} \\
\stackrel{\circ}{\circ}\end{array}$ & $\stackrel{\stackrel{n}{\xi}}{\stackrel{\circ}{\sigma}}$ & 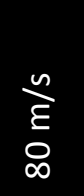 & $\stackrel{n}{\stackrel{n}{\xi}}$ & $\stackrel{\tilde{\xi}}{\stackrel{n}{\varnothing}}$ & $\begin{array}{l}\stackrel{n}{\xi} \\
\text { 유 }\end{array}$ & $\frac{\tilde{\varepsilon}}{\tilde{g}}$ & $\begin{array}{l}\stackrel{n}{\xi} \\
\stackrel{m}{m}\end{array}$ & $\begin{array}{l}\stackrel{n}{\xi} \\
\stackrel{\nu}{\nu}\end{array}$ & $\underset{\xi}{\stackrel{n}{\xi}}$ \\
\hline Aegaeon & 19 & 16 & 16 & 15 & 14 & 14 & 12 & 8 & 3 & 2 & 2 & 2 & 1 & 1 & & & & & \\
\hline Atlas & 21 & 20 & 20 & 18 & 18 & 14 & 9 & 4 & 1 & & & & & & & & & & \\
\hline Calypso & 27 & 26 & 26 & 25 & 25 & 24 & 22 & 17 & 12 & 4 & 4 & 3 & 2 & 1 & & & & & \\
\hline Daphnis & 14 & 13 & 13 & 13 & 10 & 8 & 5 & 3 & & & & & & & & & & & \\
\hline Dione & 25 & 25 & 25 & 25 & 23 & 23 & 21 & 20 & 16 & 10 & 9 & 7 & 7 & 6 & 6 & 6 & 5 & 4 & 4 \\
\hline Enceladus & 44 & 44 & 44 & 43 & 43 & 43 & 41 & 39 & 31 & 23 & 22 & 22 & 20 & 19 & 19 & 19 & 18 & 13 & 12 \\
\hline Epimetheus & 22 & 22 & 21 & 21 & 21 & 16 & 13 & 11 & 2 & 1 & 1 & 1 & 1 & 1 & & & & & \\
\hline Helene & 26 & 25 & 25 & 25 & 24 & 24 & 23 & 21 & 16 & 7 & 7 & 5 & 4 & 2 & 2 & 2 & 2 & 2 & \\
\hline Hyperion & 8 & 8 & 8 & 8 & 8 & 8 & 8 & 8 & 8 & 4 & 4 & 4 & 3 & 3 & 3 & 2 & 2 & 1 & 1 \\
\hline lapetus & 2 & 2 & 2 & 2 & 2 & 2 & 2 & 2 & 2 & 1 & 1 & 1 & 1 & 1 & 1 & 1 & 1 & 1 & 1 \\
\hline Janus & 18 & 17 & 14 & 13 & 12 & 11 & 10 & 6 & 3 & & & & & & & & & & \\
\hline Methone & 28 & 28 & 26 & 25 & 24 & 23 & 19 & 16 & 11 & 6 & 6 & 4 & 3 & 3 & 3 & 1 & 1 & & \\
\hline Mimas & 20 & 19 & 18 & 16 & 16 & 15 & 12 & 8 & 4 & 2 & 2 & 2 & 1 & 1 & 1 & 1 & & & \\
\hline Pallene & 23 & 23 & 21 & 21 & 20 & 20 & 18 & 13 & 8 & 2 & 2 & 1 & 1 & 1 & 1 & & & & \\
\hline Pan & 13 & 13 & 13 & 13 & 13 & 8 & 6 & 3 & 2 & & & & & & & & & & \\
\hline Pandora & 22 & 21 & 21 & 20 & 19 & 15 & 11 & 6 & 1 & 1 & & & & & & & & & \\
\hline Phoebe & 1 & 1 & 1 & 1 & 1 & 1 & 1 & 1 & 1 & 1 & 1 & 1 & 1 & 1 & 1 & 1 & 1 & 1 & 1 \\
\hline Polydeuces & 14 & 14 & 14 & 14 & 14 & 14 & 14 & 11 & 7 & 4 & 4 & 4 & 3 & 2 & & & & & \\
\hline Prometheus & 27 & 26 & 26 & 24 & 21 & 19 & 13 & 8 & 3 & & & & & & & & & & \\
\hline Rhea & 21 & 21 & 21 & 20 & 19 & 17 & 17 & 17 & 14 & 10 & 9 & 8 & 7 & 7 & 7 & 7 & 6 & 5 & 5 \\
\hline Telesto & 31 & 29 & 29 & 27 & 27 & 25 & 24 & 20 & 16 & 8 & 8 & 8 & 8 & 7 & 5 & 3 & 2 & 2 & \\
\hline Tethys & 27 & 27 & 27 & 27 & 27 & 25 & 20 & 19 & 16 & 7 & 7 & 5 & 4 & 3 & 3 & 3 & 2 & & \\
\hline Titan & 161 & 159 & 158 & 157 & 157 & 155 & 148 & 146 & 142 & 141 & 139 & 139 & 138 & 138 & 137 & 134 & 133 & 127 & 96 \\
\hline Grand Total & 614 & 599 & 589 & 573 & 558 & 524 & 469 & 407 & 319 & 234 & 228 & 217 & 205 & 197 & 189 & 180 & 173 & 156 & 120 \\
\hline
\end{tabular}

Orbiter Data

\begin{tabular}{|c|c|c|c|c|c|}
\hline Name & $5 \mathrm{~km} / \mathrm{s}$ & $4 \mathrm{~km} / \mathrm{s}$ & $3 \mathrm{~km} / \mathrm{s}$ & $2 \mathrm{~km} / \mathrm{s}$ & $1 \mathrm{~km} / \mathrm{s}$ \\
\hline Hyperion & 3 & & & & \\
\hline lapetus & 2 & 2 & 2 & 2 & \\
\hline \multicolumn{6}{|l|}{ Phoebe } \\
\hline Rhea & 1 & & & & \\
\hline Titan & 177 & 171 & 117 & 7 & 4 \\
\hline Grand Total & 183 & 173 & 119 & 9 & 4 \\
\hline
\end{tabular}

